\title{
Intrinsic ultracontractivity via capacitary width
}

\author{
Hiroaki Aikawa
}

Abstract. The semigroup associated with the Dirichlet heat kernel is said to be intrinsic ultracontractive if

(i) the Dirichlet realization of the associated self adjoint operator has the first positive eigenvalue with positive $L^{2}$ eigenfunction;

(ii) the heat kernel is bounded above and below by the product of the eigenfunctions with positive multiplicative constants depending on time.

We give an upper and lower estimate of the first eigenvalue in terms of capacitary width, which yields a sharp sufficient condition for (i). Our parabolic argument also yields an exponential decay property of a certain caloric measure. Then, the caloric measure is controlled by the elliptic Green function with the aid of a parabolic box argument. This is a crucial step for (ii). We give a sharp sufficient integral condition for intrinsic ultracontractivity in terms of capacitary width. A similar integral condition for the boundary Harnack principle is also obtained. Under geometric specifications, these integral conditions generalize known results and give more precise conditions. Sharpness is examined by an infinite funnel, for which we obtain a complete characterization of intrinsic ultracontractivity. Our method is purely analytic and elementary; it enables us to dispense with logarithmic Sobolev inequalities.

\section{Introduction}

\subsection{Main results}

Let $D$ be a domain in $\mathbb{R}^{n}, n \geq 2$. Let $\mathcal{L}$ be a uniformly elliptic operator in divergence form on $D$, i.e.,

$$
\mathcal{L} f(x)=\sum_{i, j=1}^{n} \frac{\partial}{\partial x_{i}}\left(a_{i, j}(x) \frac{\partial f}{\partial x_{j}}\right)(x),
$$

Mathematics Subject Classification (2010): 35K08,31B15, 31B25, 31B35.

Keywords: Intrinsic ultracontractivity, first eigenvalue, heat kernel, capacitary width, boundary Harnack principle, box argument, quasihyperbolic metric. 
where $a(x)=\left(a_{i j}(x)\right)$ is a smooth symmetric $n \times n$ matrix satisfying

(1.1) $c_{\mathcal{L}}^{-1}|\xi|^{2} \leq \sum_{i, j=1}^{n} a_{i j}(x) \xi_{i} \xi_{j} \leq c_{\mathcal{L}}|\xi|^{2}$ for all $x \in \mathbb{R}^{n}$ and $\xi=\left(\xi_{1}, \ldots, \xi_{n}\right) \in \mathbb{R}^{n}$,

with $1<c_{\mathcal{L}}<\infty$. A solution $u$ to the parabolic equation $(\mathcal{L}-\partial / \partial t) u=0$ is referred to as an $\mathcal{L}$-caloric function. For simplicity we suppress the prefix " $\mathcal{L}$-" and we say caloric for $\mathcal{L}$-caloric. Similarly, harmonic means $\mathcal{L}$-harmonic, unless otherwise stated. The difference between $\mathcal{L}$ and $\Delta$ will be illustrated in the appendix. Let $p_{D}(t, x, y), t>0, x, y \in D$, be the heat kernel for $\mathcal{L}-\partial / \partial t$ on $D$, i.e., the fundamental solution to $(\mathcal{L}-\partial / \partial t) u=0$ subject to the Dirichlet boundary condition $u(t, x)=0$ for $x \in \partial D$ and $t>0$. We have the semigroup property

$$
p_{D}(s+t, x, y)=\int_{D} p_{D}(s, x, z) p_{D}(t, z, y) d z \quad \text { for all } s, t>0 \text { and } x, y \in D .
$$

Definition 1.1. We say that the semigroup associated with $p_{D}(t, x, y)$ is intrinsically ultracontractive (abbreviated to IU) if the following two conditions are satisfied:

(i) The eigenvalue problem $-\mathcal{L} u=\lambda u$ in $D$ subject to the Dirichlet boundary condition $u=0$ on $\partial D$ has the first eigenvalue $\lambda_{D}>0$ with corresponding positive eigenfunction $\varphi_{D}$ normalized by $\left\|\varphi_{D}\right\|_{2}=1$. $\left(\varphi_{D}\right.$ is referred to as the ground state.)

(ii) For every $t>0$, there exist constants $0<c_{t}<1<C_{t}$ depending on $t$ such that

$$
c_{t} \varphi_{D}(x) \varphi_{D}(y) \leq p_{D}(t, x, y) \leq C_{t} \varphi_{D}(x) \varphi_{D}(y) \text { for all } x, y \in D
$$

For simplicity, we say that $D$ itself is IU if the semigroup associated with $p_{D}(t, x, y)$ is IU.

The purpose of this paper is to give several sharp sufficient conditions for IU by using capacitary width, which was introduced in [1] in connection with the Cranston-McConnell inequality, an intimate property with IU. Capacitary width was also useful for the elliptic boundary Harnack principle ([2], [4] and [5]). It also plays a crucial role for IU via a parabolic box argument, a counterpart of the box argument used for the boundary Harnack principle ([11]). We shall observe that IU and the boundary Harnack principle can be dealt with in a unified fashion with capacitary width. Our results are new even if $\mathcal{L}$ is the Laplacian $\Delta$.

For an open set $U$ we consider a quadratic form

$$
Q_{U}[\varphi]=\int_{U} \sum_{i, j=1}^{n} a_{i j}(x) \frac{\partial \varphi}{\partial x_{i}} \frac{\partial \varphi}{\partial x_{j}} d x
$$

For $E \subset U$ we define capacity associated with $\mathcal{L}$ by

$$
\operatorname{Cap}_{U}^{\mathcal{L}}(E)=\inf \left\{Q_{U}[\varphi]: \varphi \geq 1 \text { on } E, \varphi \in C_{0}^{\infty}(U)\right\} .
$$


Let $G_{U}^{\mathcal{L}}(x, y)$ be the Green function for $\mathcal{L}$, normalized by $-\mathcal{L} G_{U}^{L}(\cdot, y)=\delta_{y}$. Observe that $\operatorname{Cap}_{U}^{\mathcal{L}}(E)$ coincides with the Green capacity of $E$ with respect to $U$, i.e.,

$$
\operatorname{Cap}_{U}^{\mathcal{L}}(E)=\sup \left\{\|\mu\|: \operatorname{supp} \mu \subset E \text { and } \int_{U} G_{U}^{\mathcal{L}}(x, y) d \mu(y) \leq 1 \text { on } D\right\} .
$$

Let us write $\operatorname{Cap}_{U}(E)$ if $\mathcal{L}$ is the Laplacian $\Delta$. In view of (1.1) we have

$$
c_{\mathcal{L}}^{-1} \operatorname{Cap}_{U}(E) \leq \operatorname{Cap}_{U}^{\mathcal{L}}(E) \leq c_{\mathcal{L}} \operatorname{Cap}_{U}(E) .
$$

We write $B(x, r)$ for the open ball with center at $x$ and radius $r$.

Definition 1.2. Let $0<\eta<1$. For an open set $D$ we define the capacitary width $w_{\eta}(D)$ as

$$
w_{\eta}(D)=\inf \left\{r>0: \frac{\operatorname{Cap}_{B(x, 2 r)}(B(x, r) \backslash D)}{\operatorname{Cap}_{B(x, 2 r)}(B(x, r))} \geq \eta \quad \text { for all } x \in D\right\} .
$$

Remark 1.3. The value of $\eta$ has no significance. In fact, if $0<\eta<\eta^{\prime}<1$, then we have

$$
w_{\eta}(D) \leq w_{\eta^{\prime}}(D) \leq A w_{\eta}(D) \text { for every open set } D,
$$

where $A>1$ depends only on $\eta, \eta^{\prime}$ and $n$. See Proposition 2 in [1] or Lemma 12.8 in the appendix.

Remark 1.4. We can replace

$$
\frac{\operatorname{Cap}_{B(x, 2 r)}(B(x, r) \backslash D)}{\operatorname{Cap}_{B(x, 2 r)}(B(x, r))} \text { by } \frac{\operatorname{Cap}_{B(x, 2 r)}(\bar{B}(x, r) \backslash D)}{\operatorname{Cap}_{B(x, 2 r)}(\bar{B}(x, r))} \text {. }
$$

This has an advantage that $E=\bar{B}(x, r) \backslash D$ and $\bar{B}(x, r)$ are compact sets, so that we can consider the capacitary potentials of $E$ and $\bar{B}(x, r)$ in $B(x, 2 r)$. See Remark 12.4 for details. Gyrya and Saloff-Coste [21] adopted this definition. For capacitary potential, capacity and related subjects, we refer to Chapter 5 in [7].

Let us begin with an estimate of $\lambda_{D}$. Note that $\lambda_{D}$ is characterized by the variational problem:

$$
\lambda_{D}=\inf \left\{\frac{Q_{D}[\varphi]}{\|\varphi\|_{2}^{2}}: \varphi \in C_{0}^{\infty}(D)\right\} .
$$

If $\mathcal{L}$ is the Laplacian, then $\lambda_{D}$ is referred to as the principal frequency of $D$. There are a number of papers dealing with the principal frequency. Very precise estimates are known under geometrical hypothesis on $D$. We present an estimate not so precise but applicable to arbitrary domains.

Theorem 1.5. We have

$$
\frac{A^{-1}}{w_{\eta}(D)^{2}} \leq \lambda_{D} \leq \frac{A}{w_{\eta}(D)^{2}}
$$

where $A>1$ depends only on $c_{\mathcal{L}}, \eta$ and $n$. In particular, $\lambda_{D}>0$ if and only if $w_{\eta}(D)<\infty$. 
Remark 1.6. Maz'ya-Shubin [26] gave the same estimate with $r_{D, \gamma}$ in place of $w_{\eta}(D)$. They defined $r_{D, \gamma}$ as the supremum of radius $r$ for which there exists an open ball $B_{r}$ of radius $r$ such that $\bar{B}_{r} \backslash D$ is $\gamma$-negligible with $0<\gamma<1$, i.e., the Wiener capacity of $\bar{B}_{r} \backslash D$ is less than $\gamma$ times of that of $\bar{B}_{r}$. It is easy to see that $r_{D, \gamma}$ is comparable to our capacitary width $w_{\eta}(D)$.

With the aid of Persson's argument [34], we see that the bottom of the essential spectrum of $-\mathcal{L}$ is $+\infty$ if and only if

$$
\lim _{R \rightarrow \infty} w_{\eta}(D \backslash \bar{B}(0, R))=0 .
$$

In particular, we obtain the following.

Corollary 1.7. If (1.6) holds, then Definition 1.1 (i) is valid.

It is known that if $D$ is IU, then $-\mathcal{L}$ has only purely discrete spectrum. So, we can replace Definition 1.1 (i) by (1.6). We shall present a proof of Theorem 1.5 in the parabolic context, which is inspired by Souplet [37]. Our approach is shorter and more transparent than Maz'ya-Shubin [26]. In the course of the proof, we naturally obtain useful information about the heat kernel, which plays a crucial role in the study of Definition 1.1(ii). For instance, a decay estimate of

$$
P(t, x, D)=\int_{D} p_{D}(t, x, y) d y
$$

will be derived. Observe that $P(t, x, D)$ is the caloric function on the cylinder $(0, \infty) \times D$ with initial condition $P(0, x, D)=1$ for $x \in D$ and lateral boundary condition $P(t, x, D)=0$ for $x \in \partial D$. We can regard $P(t, x, D)$ as a caloric measure, a parabolic counterpart of harmonic measure. Obviously, $0 \leq P(t, x, D) \leq 1$. We shall observe in Section 3 that if $w_{\eta}(D)<\infty$, then it decays exponentially. More precisely, we obtain the following proposition.

Proposition 1.8. There exist positive constants $A_{0}$ and $A_{1}$ depending only on $c_{\mathcal{L}}, \eta$ and $n$ such that

$$
P(t, x, D) \leq A_{0} \exp \left(-\frac{A_{1} t}{w_{\eta}(D)^{2}}\right) \quad \text { for all } t>0 \text { and } x \in D .
$$

Hereafter, we assume that (1.6) holds. Verification of Definition 1.1 (ii) is still subtle under this assumption. The validity of Definition 1.1 (ii) requires some regularity of $D$, although it is very mild. The notion of IU was introduced by Davies-Simon [17]. IU has several equivalent conditions and enjoys many interesting properties in functional analysis and in probability theory. See [16] for analytic aspects. IU has important consequences in the study of the lifetime of conditioned Brownian motions. Many authors are fascinated by IU and have given a number of contributions. See e.g. [8], [9], [12], [15], [18], [24], [27], and so on. Nowadays, IU for general processes is extensively studied from a probabilistic point of view. Murata gave many interesting applications of IU to perturbations of Green functions, Martin boundaries and the heat equation on manifolds. See [28], [29], [30], [31], [32], and references therein. 
There are basically two different general methods available to prove sufficient conditions for IU. The first is based on the theory of logarithmic Sobolev inequalities ([8], [15],[17], and [27]). The second is based on handcrafted probabilistic work (Bass-Burdzy [12]).

In this paper, we propose a new approach, a parabolic box argument, which is a parabolic counterpart of the box argument invented by Bass-Burdzy [11] for the boundary Harnack principle for harmonic functions. We can treat IU and the boundary Harnack principle in a unified fashion. We shall show several sufficient conditions for IU and the boundary Harnack principle in terms of capacitary width.

Let us state our results for Definition 1.1(ii). For simplicity let us suppress scripts and write simply $p(t, x, y)$ and $G(x, y)$ for the heat kernel and the Green function for $\mathcal{L}$ in $D$. For a generic positive function $f(x)$ on $D$ and $t>0$ we write $w_{\eta}(f<t)=w_{\eta}(\{x \in D: f(x)<t\})$.

Theorem 1.9. Let $g=G\left(\cdot, x_{0}\right)$ with $x_{0} \in D$. If

$$
\int_{0}^{1} w_{\eta}(g<t)^{2} \frac{d t}{t}<\infty
$$

then $D$ is IU. Moreover, for each $\varepsilon>0$, the constant $C_{t}$ in (1.2) has an estimate

$$
C_{t} \leq \frac{A_{2}}{m^{2+\varepsilon}} t^{-n / 2}
$$

with $m>0$ being chosen so that

$$
\int_{0}^{m} w_{\eta}(g<s)^{2} \frac{d s}{s}<A_{3} t,
$$

where the positive constants $A_{2}$ and $A_{3}$ depend only on $c_{\mathcal{L}}, n, \eta$ and $\varepsilon$.

Sharper estimates of $C_{t}$ will be obtained under more specific conditions. See Propositions 5.5, 7.4, 8.2, and 9.7. Theorem 1.9 has a counterpart for the global boundary Harnack principle.

Definition 1.10. We say that $D$ enjoys the global boundary Harnack principle (abbreviated to GBHP) if for each pair $(V, K)$ of a bounded open set $V \subset \mathbb{R}^{n}$ and a compact set $K \subset \mathbb{R}^{n}$ such that

$$
K \subset V, \quad K \cap D \neq \emptyset \text { and } K \cap \partial D \neq \emptyset,
$$

we have the following property: if $u$ and $v$ are positive superharmonic functions in $D$ such that

(i) $u$ and $v$ are bounded, positive and harmonic in $V \cap D$,

(ii) $u$ and $v$ vanish on $V \cap \partial D$ outside a polar set,

then

$$
\frac{u(x) / u(y)}{v(x) / v(y)} \leq A \quad \text { for } x, y \in K \cap D,
$$

where $A$ depends only on $D, V$ and $K$. 
The GBHP can be reformulated in a fashion reminiscent of IU. In view of Theorem 2.3 in [4], we see that $D$ enjoys the GBHP if and only if

$$
A^{-1} g(x) g(y) \leq G(x, y) \leq A g(x) g(y) \quad \text { for } x \in K \cap D \text { and } y \in F \cap D
$$

whenever $K$ and $F$ are disjoint compact sets intersecting $D$ such that $x_{0} \notin K \cup F$. For the reader's convenience we shall provide a proof of this equivalence (Proposition 2.4). Let us give an elliptic counterpart of Theorem 1.9.

Theorem 1.11. If

$$
\int_{0}^{1} w_{\eta}(g<t) \frac{d t}{t}<\infty
$$

then $D$ satisfies the GBHP.

Remark 1.12. Compare (1.2) and (1.12). The difference of these estimates comes from the difference of parabolic and elliptic dilation exponents. Note also that the Green function $G(x, y)$ diverges on the diagonal $x=y$, whereas the heat kernel is continuous on the diagonal if $t>0$. Philosophically, $G(x, y)$ is estimated by reducing the range of points $x$ and $y$ (the elliptic box argument); while $p(t, x, y)$ is estimated by reducing the range of time $t$ (the parabolic box argument). See Sections 4 and 6.

Remark 1.13. In view of Definition 1.10, we see that the GBHP is a property of bounded nature, i.e., $D$ enjoys the GBHP if and only if $D \cap V$ enjoys the GBHP for every bounded open set $V$. Hence we can relax (1.13) to

$$
\int_{0}^{1} w_{\eta}\left(\left\{x \in D \cap V: G_{D \cap V}\left(x, x_{0}\right)<t\right\}\right) \frac{d t}{t}<\infty
$$

for every bounded open set $V$ containing $x_{0}$.

Remark 1.14. There is another type of boundary Harnack principle, which is usually referred to as the local boundary Harnack principle (LBHP) or the scaleinvariant boundary Harnack principle. The LBHP is a much stronger property; it holds for Lipschitz domains, NTA domains and, more generally, uniform domains. The LBHP characterizes uniform domains. See [2] and [3].

\subsection{Applications}

Theorems 1.9 and 1.11 yield many sufficient conditions for IU and the GBHP. We can give more geometric conditions not involving $g$. Define the quasihyperbolic metric $k_{D}(x, y)$ as

$$
k_{D}(x, y)=\inf _{\gamma} \int_{\gamma} \frac{d s}{\delta_{D}(\gamma(s))} \quad \text { for } x, y \in D
$$


where the infimum is taken over all rectifiable curves $\gamma$ connecting $x$ and $y$ in $D ; \gamma$ is parameterized as $\gamma(s), 0 \leq s \leq \ell(\gamma)$, by arc length $s$ with $\ell(\gamma)$ being the length of $\gamma$. If $h$ is a positive harmonic function in $D$, then

$$
\exp \left(-A k_{D}(x, y)\right) \leq \frac{h(y)}{h(x)} \leq \exp \left(A k_{D}(x, y)\right) \quad \text { for } x, y \in D,
$$

where $A>0$ depends only on $c_{\mathcal{L}}$ and $n$, since the shortest length of Harnack chains connecting $x$ and $y$ in $D$ is estimated by the quasihyperbolic distance $k_{D}(x, y)$. Since $g$ is a positive harmonic function in $D \backslash\left\{x_{0}\right\}$, it follows that $g\left(x_{0}^{\prime}\right) / g(x) \leq \exp \left(A k_{D}\left(x, x_{0}\right)\right)$, where $x_{0}^{\prime} \in \partial B\left(x_{0}, \delta_{D}\left(x_{0}\right) / 2\right)$. Hence we obtain $g(x) \geq \exp \left(-A k_{D}\left(x, x_{0}\right)\right)$, in other words,

$$
k_{D}\left(x, x_{0}\right) \geq A \log (1 / g(x)) .
$$

with $A$ changed. In particular, $\{x \in D: g(x)<t\} \subset\left\{x \in D: k_{D}\left(x, x_{0}\right)>\right.$ $A \log (1 / t)\}$. By the change of variable $s=A \log (1 / t)$ we obtain the following immediate corollary to Theorems 1.9 and 1.11 .

Corollary 1.15. The following statements hold:

(i) If $\int_{0}^{\infty} w_{\eta}\left(k_{D}\left(\cdot, x_{0}\right)>s\right)^{2} d s<\infty$, then $D$ is $I U$.

(ii) If $\int_{0}^{\infty} w_{\eta}\left(k_{D}\left(\cdot, x_{0}\right)>s\right) d s<\infty$, then $D$ satisfies the GBHP.

Let us give an Orlicz type condition. Let $\Phi(t)$ be a positive nondecreasing continuous function of $t>0$ with $\Phi(0)=0$ and let

$$
L^{\Phi}(D)=\left\{f: \int_{D} \Phi(|f(x)|) d x<\infty\right\} .
$$

If $\Phi(t)=t^{p}$, then $L^{\Phi}(D)$ coincides with the usual $L^{p}$-space $L^{p}(D)$. We do not discuss properties of $L^{\Phi}(D)$ as a function space, so we do not assume any further hypothesis on $\Phi$ such as convexity. Let $\log _{+}(u)=\max \{\log u, 0\}$ for $u>0$.

Theorem 1.16. The following statements hold:

(i) Let $n=2$. If $\log _{+}(1 / g) \in L^{1}(D)$, then $D$ is $I U$.

(ii) Let $n \geq 3$. Suppose

$$
\int_{1}^{\infty}\left(\frac{t}{\Phi(t)}\right)^{2 /(n-2)} d t<\infty
$$

If $\log _{+}(1 / g) \in L^{\Phi}(D)$, then $D$ is $I U$.

(iii) Let $n \geq 2$. Suppose

$$
\int_{1}^{\infty}\left(\frac{t}{\Phi(t)}\right)^{1 /(n-1)} d t<\infty
$$

If $\log _{+}(1 / g) \in L^{\Phi}(D)$, then $D$ satisfies the GBHP. 
Remark 1.17. (i) Let $n \geq 3$. Typical examples of $\Phi(t)$ satisfying (1.16) are $\Phi(t)=t^{p}$ with $p>n / 2$ and $\Phi(t)=t^{n / 2} \log ^{\alpha}(e+t)$ with $\alpha>(n-2) / 2$. See Theorem 3 in [15]. See Proposition 7.4 for an estimate of $C_{t}$ in (1.2).

(ii) Let $n \geq 2$. Typical examples of $\Phi(t)$ satisfying (1.17) are $\Phi(t)=t^{p}$, with $p>n$, and $\Phi(t)=t^{n} \log ^{\alpha}(e+t)$ with $\alpha>n-1$.

With the aid of (1.15), we have an immediate corollary to Theorem 1.16.

Corollary 1.18. The following statements hold:

(i) Let $n=2$. If $k_{D}\left(\cdot, x_{0}\right) \in L^{1}(D)$, then $D$ is IU.

(ii) Let $n \geq 3$ and suppose $\Phi$ satisfies (1.16). If $k_{D}\left(\cdot, x_{0}\right) \in L^{\Phi}(D)$, then $D$ is IU (cf. Theorem 6 in [15]).

(iii) Let $n \geq 2$ and suppose $\Phi$ satisfies (1.17). If $k_{D}\left(\cdot, x_{0}\right) \in L^{\Phi}(D)$, then $D$ satisfies the GBHP.

Remark 1.19. The sufficient conditions for the GBHP in Theorem 1.16 and Corollary 1.18 are new even if $\Phi(t)=t^{p}$ with $p>n$.

Let us consider families of domains defined by conditions in terms of the quasihyperbolic metric. Smith-Stegenga ([35] and [36]) said that D is a "Hölder domain" if

$$
k_{D}\left(x, x_{0}\right) \leq A \log \frac{\delta_{D}\left(x_{0}\right)}{\delta_{D}(x)}+A^{\prime} \quad \text { for all } x \in D,
$$

with some positive constants $A$ and $A^{\prime}$. Bañuelos [8] called such a domain a Hölder domain of order 0 . However, the term "Hölder domain" is often used for a domain whose boundary is given by the graph of a Hölder continuous function. To avoid the confusion, we say that $D$ satisfies the quasihyperbolic boundary condition (of order 0) (abbreviated to QHB(0)) if (1.18) holds. Extending (1.18), we consider the following condition for $\alpha>0$ :

$$
k_{D}\left(x, x_{0}\right) \leq A\left(\frac{\delta_{D}\left(x_{0}\right)}{\delta_{D}(x)}\right)^{\alpha}+A^{\prime} \quad \text { for all } x \in D
$$

with some positive constants $A$ and $A^{\prime}$. Let us say that $D$ satisfies the quasihyperbolic boundary condition of order $\alpha$ (abbreviated to $\mathrm{QHB}(\alpha)$ ) if (1.19) holds.

So far, we have considered interior conditions. Let us introduce an exterior condition, which will be useful to estimate capacitary width. See Lemma 3.13.

Definition 1.20. Let $0<r_{0} \leq \infty$. We say that $D$ satisfies the capacity density condition (abbreviated to CDC) up to $r_{0}$ if there exists positive constant $\eta$ such that

$$
\frac{\operatorname{Cap}_{B(x, 2 r)}(B(x, r) \backslash D)}{\operatorname{Cap}_{B(x, 2 r)}(B(x, r))} \geq \eta
$$

whenever $x \in \partial D$ and $0<r<r_{0}$. We simply say that $D$ satisfies the CDC if it satisfies the CDC up to $r_{0}$ for some $r_{0}>0$. 
The following theorem is known:

Theorem A ([8] and [4]). Suppose D satisfies the CDC.

(i) If $D$ satisfies the $Q H B(\alpha)$ with $0 \leq \alpha<2$, then $D$ is IU.

(ii) If $D$ satisfies the $Q H B(\alpha)$ with $0 \leq \alpha<1$, then the GBHP holds.

Remark 1.21. In case $\alpha=0$, the CDC can be removed (Bañuelos [8], Theorem 1). This result can be derived from Theorem 1.16 and the integrability of the quasihyperbolic metric due to Smith-Stegenga [35] and [36], i.e., if $D$ satisfies the QHB(0), then there exists a positive constant $\tau$ such that

$$
\int_{D} \exp \left(\tau k_{D}\left(x, x_{0}\right)\right) d x<\infty
$$

or equivalently $k_{D}\left(\cdot, x_{0}\right) \in L^{\Phi}(D)$ with $\Phi(t)=\exp (\tau t)$. Obviously, $\Phi(t)=\exp (\tau t)$ satisfies (1.16) and (1.17), so that $D$ is IU and it satisfies the GBHP by Theorem 1.16.

Remark 1.22. Bass-Burdzy (Definition 2.2 in [12]) defined a twisted $L^{p}$-domain and proved that if $p>n-1$, then every twisted $L^{p}$-domain with the CDC is IU. In view of their definition, we see that $D$ is a twisted $L^{p}$-domain if and only if it satisfies the $\mathrm{QHB}((n+p-1) / p)$. Hence Bass-Burdzy's result is the same as Theorem A (i).

By using Theorems 1.9 and 1.11, we can give a further extension. Let $\Phi(t)$ be a positive nondecreasing continuous function of $t>0$. We say that $D$ satisfies the $\mathrm{QHB}(\Phi)$ condition if

$$
k_{D}\left(x, x_{0}\right) \leq \Phi\left(\frac{\delta_{D}\left(x_{0}\right)}{\delta_{D}(x)}\right) \text { for all } x \in D .
$$

Theorem 1.23. Suppose D satisfies the CDC.

(i) If $D$ satisfies the $Q H B(\Phi)$ with

$$
\int_{1}^{\infty} \Phi(t) \frac{d t}{t^{3}}<\infty
$$

then $D$ is $I U$.

(ii) If $D$ satisfies the $Q H B(\Phi)$ with

$$
\int_{1}^{\infty} \Phi(t) \frac{d t}{t^{2}}<\infty
$$

then D satisfies the GBHP.

Remark 1.24. (i) Typical examples of $\Phi(t)$ satisfying (1.21) are $\Phi(t)=t^{\alpha}$ with $\alpha<2$ and $\Phi(t)=t^{2} \log ^{-\alpha}(e+t)$ with $\alpha>1$. See Corollary 2.8 in [8]. See Proposition 8.2 for an estimate of $C_{t}$ in (1.2). 
(ii) Typical examples of $\Phi(t)$ satisfying (1.22) are $\Phi(t)=t^{\alpha}$ with $\alpha<1$, and $\Phi(t)=t \log ^{-\alpha}(e+t)$ with $\alpha>1$.

Davis [18] and Bass-Burdzy [12] studied IU for domains above the graph of a function. We write $x=\left(x^{\prime}, x_{n}\right) \in \mathbb{R}^{n}$. By $B^{\prime}\left(x^{\prime}, R\right)$ we denote the $(n-1)$ dimensional open ball with center at $x^{\prime}$ and radius $R$.

Theorem B. For a negative upper semicontinuous function $f\left(x^{\prime}\right)$ on $B^{\prime}(0, R)$, write

$$
D_{f}=\left\{\left(x^{\prime}, x_{n}\right):\left|x^{\prime}\right|<R, f\left(x^{\prime}\right)<x_{n}<1\right\} .
$$

Then we have the following assertions:

(i) If $n=2$ and $f \in L^{\infty}\left(B^{\prime}(0, R)\right)$, then $D_{f}$ is IU (Theorem 2 in [18]).

(ii) If $f \in L^{p}\left(B^{\prime}(0, R)\right)$ with $p>n-1$, then $D_{f}$ is IU (Theorem 1.22 in [12]).

(iii) If $n \geq 3$, then there exists $f \in L^{p}\left(B^{\prime}(0, R)\right)$ with $p<n-1$ such that $D_{f}$ is not IU (Section 4 in [12]).

Obviously, (i) is included in (ii). Note that $D_{f}$ can be unbounded in (ii). We remark that $D_{f}$ satisfies the quasihyperbolic boundary condition.

Proposition 1.25. If $f \in L^{p}\left(B^{\prime}(0, R)\right)$ with $p>0$, then $D_{f}$ satisfies the $Q H B((p+$ $n-1) / p$ ) condition.

It is easy to see that $(p+n-1) / p<2$ if and only if $p>n-1$. Hence, under the additional assumption of the CDC, Theorem B (ii) can be derived from Theorem A (i). The significance of Theorem B (ii) is that IU follows without the $C D C$. This remarkable phenomenon is rooted in Lemma 2.4 in [12], which is reformulated analytically as an extended Harnack inequality with exceptional sets in [5]. The critical case $p=n-1$ in Theorem B (iii) was open. Actually, we shall show in Corollary 1.31 below that there is $f \in L^{n-1}\left(B^{\prime}(0, R)\right)$ such that $D_{f}$ is not IU in case $n \geq 3$. So, let us consider a condition sharper than $f \in L^{n-1}\left(B^{\prime}(0, R)\right)$. Let $\Phi(t)$ be a positive nondecreasing function of $t>0$.

Theorem 1.26. Assume that $\Phi(t) / t^{n-1}$ is nondecreasing and that

$$
\int_{1}^{\infty} \Phi(t)^{1 /(1-n)} d t<\infty .
$$

If $f \in L^{\Phi}\left(B^{\prime}(0, R)\right)$, then $D_{f}$ is $I U$.

Remark 1.27. Typical examples of $\Phi(t)$ satisfying (1.23) are $\Phi(t)=t^{p}$ with $p>n-1$ and $\Phi(t)=t^{n-1} \log ^{\alpha}(e+t)$ with $\alpha>n-1$. See Proposition 9.7 for an estimate of $C_{t}$ in (1.2).

Theorem 1.26 can be extended to $L^{\Phi}$-domains. See Theorem 10.5. A counterpart of Theorem 1.26 for the GBHP has very different appearance since (1.13) is much more stringent than (1.8). We say that a bounded domain in $\mathbb{R}^{n}$ is a $\psi$-Hölder 
domain if its boundary is locally given by the graph of a $\psi$-Hölder continuous function in $\mathbb{R}^{n-1}$. We showed an upper estimate of capacitary width

$$
w_{\eta}(g<t) \leq \frac{A}{\log (1 / t)} \psi\left(\frac{1}{\log (1 / t)}\right) \text { for small } t>0,
$$

provided that $\limsup _{t \rightarrow 0} \psi(M t) / \psi(t)<M$ with some $M>1$ (Corollary 7.7 in [5]). Since

$$
\int_{0}^{1} w_{\eta}(g<t) \frac{d t}{t} \leq \int_{0}^{1 / e} \frac{1}{\log (1 / t)} \psi\left(\frac{1}{\log (1 / t)}\right) \frac{d t}{t}=\int_{0}^{1} \frac{\psi(t)}{t} d t
$$

by a change of variable, Theorem 1.11 gives the following immediate corollary.

Theorem C (Theorem 1.3 in [5]). Let $\psi(t)$ be a nondecreasing continuous function for $t>0$. Suppose that $\psi$ satisfies $\limsup _{t \rightarrow 0} \psi(M t) / \psi(t)<M$ for some $M>1$ and

$$
\int_{0}^{1} \frac{\psi(t)}{t} d t<\infty
$$

Then every $\psi$-Hölder domain satisfies the GBHP.

So far, we have studied sufficient conditions for IU and the GBHP to hold. For some specific type of domains, we can determine whether these conditions are sharp, and even further, we can obtain characterizations for IU. BañuelosDavis [9] gave a geometrical characterization of intrinsic ultracontractivity for planar domains with boundaries given by the graphs of functions. Let us give a characterization of intrinsic ultracontractivity for a solid of rotation of a Lipschitz graph, which may be visualized as an infinite funnel. This is inspired by the counterexample of Bass-Burdzy (Section 4 in [12]).

Theorem 1.28. Let $L>0$ and let $r(t)$ be a positive nonincreasing L-Lipschitz function of $t \in[-1, \infty)$, i.e.,

$$
0 \leq r(t)-r(T) \leq L(T-t) \quad \text { for }-1 \leq t<T<\infty .
$$

Define an infinite funnel or a solid of rotation by

$$
V=\left\{\left(x^{\prime}, x_{n}\right):-\infty<x_{n}<1,\left|x^{\prime}\right|<r\left(-x_{n}\right)\right\} .
$$

See Figure 1. Then the following statements are equivalent:

(i) $V$ is $I U$.

(ii) V satisfies the Cranston-McConnell inequality, i.e.,

$$
\sup _{x \in V} \frac{1}{u(x)} \int_{V} G_{V}(x, y) u(y) d y \leq A
$$

for every superharmonic function $u>0$ in $V$, where $A$ is independent of $u$. 


$$
\begin{aligned}
& \text { (iii) } \int_{0}^{\infty} r(t) d t<\infty \\
& \text { (iv) } \int_{0}^{\infty} w_{\eta}\left(k_{V}\left(\cdot, x_{0}\right)>s\right)^{2} d s<\infty . \\
& \text { (v) } \int_{0}^{1} w_{\eta}(g<t)^{2} \frac{d t}{t}<\infty
\end{aligned}
$$

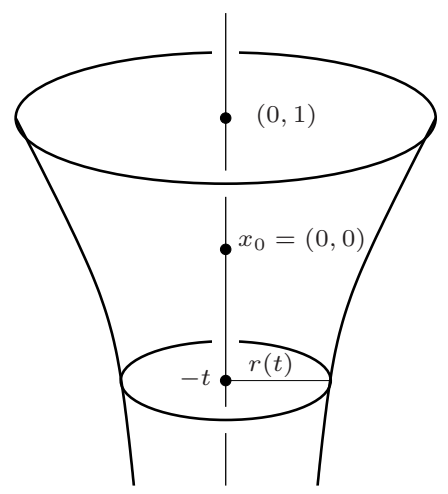

Figure 1. Infinite funnel.

Remark 1.29. Because of its local nature we can relax (1.24) into

$$
0 \leq r(t)-r(T) \leq L(T-t) \text { for } t_{1} \leq t<T<\infty
$$

with some $t_{1}>-1$.

Corollary 1.30. Let $r(t)=(t+3)^{-1}$. Then $V$ satisfies the $Q H B(2)$ and yet $V$ is not $I U$.

Corollary 1.31. Let $n \geq 3$ and let $r(t)=(t+3)^{-1} \log ^{-\alpha}(t+3)$ with $(n-1)^{-1}<$ $\alpha \leq 1$. Then $V$ is not $I U$ and yet $V$ is represented as $D_{f}=\left\{\left(x^{\prime}, x_{n}\right):\left|x^{\prime}\right|<\right.$ $\left.r(-1), x_{n}>f\left(x^{\prime}\right)\right\}$ with $f \in L^{n-1}\left(B^{\prime}(0, r(-1))\right)$.

Remark 1.32. The sharpness of sufficient conditions for the GBHP is much more delicate. It will be treated elsewhere. Actually, the infinite funnel $V$ in Theorem 1.28 is irrelevant for a test of the GBHP. The infinite funnel $V$ always satisfies the GBHP, no matter how slowly $r(t)$ decays at infinity. See Remark 1.13.

\subsection{Plan of the paper}

In Section 2 we shall provide basic material for IU in order to increase the reader's familiarity with IU. We include several basic properties of IU such as an estimate of $C_{t}$ and the implication: "the upper estimate $\Longrightarrow$ the lower estimate". It is 
well known for probabilists that "IU $\Longrightarrow$ the Cranston-McConnell inequality". However, we include an elementary analytic proof, as we have not been able to find a good reference in the literature except for Theorem 2.8 in [24], which deals with the rather general nonsymmetric case. We also provide a proof of "(1.12) the GBHP."

In Section 3 we shall prove Theorem 1.5 as a corollary to more general result (Theorem 3.1), which also yields (1.7). This estimate will be crucial in the parabolic box argument. We also exploit comparison of capacitary width with other quantities such as volumetric width and inradius. With the aid of this comparison, we shall apply Theorems 1.9 and 1.11 to specific domains and obtain more explicit sufficient conditions for IU and the GBHP later in Sections 7-11. We also observe that (1.6) is not preserved by taking the union of two domains. Section 4 is devoted to the parabolic box argument, which controls the caloric measure $P(t, x, D)$, with the aid of the upper estimate of (1.7). In Section 5 we shall prove Theorem 1.9. The comparison between the ground state and the Green function is also obtained. Section 6 is devoted to the elliptic box argument and the proof of Theorem 1.11. The main idea was already given in the previous paper [5]. These sections do not depend on the geometry of the domain $D$.

In Sections $7-11$ we shall specify the geometry of the domain $D$ and we shall give more precise conditions for IU and the GBHP. In Section 7 we give sufficient integral conditions of Orlicz type in terms of the Green function and the quasihyperbolic metric. Theorem 1.16 will be proved. In Section 8 we give sufficient conditions by pointwise upper estimates of the quasihyperbolic metric for domains satisfying the CDC. Theorem 1.23 will be proved. In Section 9 we shall dispense with the CDC and study $D_{f}$ with $f \in L^{\Phi}\left(B^{\prime}(0, R)\right)$. It will require the extended Harnack inequality with exceptional sets (Lemma 2.4 in [12] and [5]). We note that the results in this section work only for IU. The GBHP requires much more stringent geometrical hypothesis. In Section 10 we shall study pasting domains and introduce $L^{\Phi}$-domains. In Section 11, we shall obtain Theorem 1.28, a complete characterization of IU for an infinite funnel. We shall give precise estimates for the quasihyperbolic metric. We shall employ a scale invariant boundary Harnack principle to give a sharp estimate of the product of the Green function and the Martin kernel at infinity; in fact, the Martin boundary of the infinite funnel is homeomorphic to the union of the Euclidean boundary and the point at infinity. We are inspired by Hansen [22] for a counterexample of the $3 \mathrm{G}$-inequality in $\mathbb{R}^{n}$, $n \geq 3$. Theorem 1.28 asserts that our sufficient conditions are sharp.

Our arguments are based on the $\mathcal{L}$-harmonic versions of several results in [1]. Although the $\mathcal{L}$-harmonic counterparts are essentially the same as the classical ones, they require more careful arguments. So, the appendix is devoted to such generalizations for the reader's convenience. In particular, we shall replace the usage of balayage measures in Proof of Lemma 5 in [1] by a uniform estimate of capacity of balls (Lemma 12.1).

We shall freely use basic results from potential theory. For accounts on potential theory, see [7], [10], [19] and [38]. We frequently use the elliptic and parabolic comparison principles. Let $D$ be an arbitrary open set in $\mathbb{R}^{n}$ with $w_{\eta}(D)<\infty$. Since $D$ 
is arbitrary, it may be irregular with respect to the Dirichlet problem. However, irregular boundary points of $D$ forms a polar set, so that we have the following elliptic comparison principle of Phragmén-Lindelöf type: Suppose $u$ (resp. $v$ ) is a bounded subharmonic (resp. superharmonic) function in $D$. If $u \leq v$ on $\partial D$ outside a polar set, i.e.,

$$
\limsup _{x \rightarrow \xi} u(x) \leq \liminf _{x \rightarrow \xi} v(x) \quad \text { for q.e. } \xi \in \partial D,
$$

then $u \leq v$ in $D$. Here, 'q.e.' (quasi-everywhere) means that the property holds outside a polar set. Note that $w_{\eta}(D)<\infty$ is used for the control at infinity (see Lemma E). The parabolic comparison principle is as follows: suppose $u$ (resp. $v$ ) is a bounded subcaloric (resp. supercaloric) function on the cylinder $(s, t) \times D$. If $u \leq$ $v$ on $(\{s\} \times D) \cup((s, t) \times(\partial D \backslash E))$ with a polar set $E$ in $\mathbb{R}^{n}$, then $u \leq v$ on $(s, t) \times D$. See Theorem 8.2 in [38]. As a result of the parabolic comparison principle, the Dirichlet heat kernel $p_{D}(t, x, y)$ is increasing with respect to $D$. Moreover, $p(t, x, y)$ is majorized by the heat kernel $p_{\mathbb{R}^{n}}(t, x, y)$ for the whole space $\mathbb{R}^{n}$. Recall the wellknown estimate:

$$
p_{\mathbb{R}^{n}}(t, x, y) \asymp \frac{1}{t^{n / 2}} \exp \left(-\frac{|x-y|^{2}}{t}\right),
$$

i.e., there are positive constants $A$ and $A^{\prime}$ depending only on the ellipticity constant $c_{\mathcal{L}}$ and $n$ such that

$$
p_{\mathbb{R}^{n}}(t, x, y) \leq \frac{A}{t^{n / 2}} \exp \left(-A^{\prime} \frac{|x-y|^{2}}{t}\right)
$$

and the opposite inequality holds with different $A$ and $A^{\prime}$. Hence, in particular,

$$
p(t, x, y) \leq \frac{A}{t^{n / 2}} \exp \left(-A^{\prime} \frac{|x-y|^{2}}{t}\right) .
$$

Of course, there is no good lower estimate for $p(t, x, y)$ in general. See [20]. Note also that the Dirichlet Green function $G(x, y)$ exists and it is represented by the following well-known identity:

$$
G(x, y)=\int_{0}^{\infty} p(t, x, y) d t
$$

See e.g. [19], Part 1. Chapter XVII. Section 18. In fact, (1.26) holds for every domain if $n \geq 3$; it holds for $D$ with $\mathbb{R}^{2} \backslash D$ being non-polar if $n=2$.

We use the following notation. By the symbol $A$ we denote an absolute positive constant whose value is unimportant and may change from one occurrence to the next. If necessary, we use $A_{0}, A_{1}, \ldots$, to specify them. We say that two positive quantities $f$ and $g$ are comparable, and write $f \approx g$, if they satisfy $A^{-1} \leq f / g \leq A$ with some constant $A \geq 1$. The constant $A$ is referred to as the constant of comparison. We have to pay attention for the dependency of the constant of comparison.

Acknowledgments. The author would like to thank Minoru Murata for valuable discussions, and Masaharu Nishio for the opportunity to lecture on the contents of this paper at Osaka City University in June 2013. The author is grateful to the referee for his careful reading of the manuscript and many useful suggestions. 


\section{Background}

Let us provide basic material for IU. Although the results are well known, our elementary proofs may profit the reader. We need neither deep functional analysis nor probability theory. We basically use the comparison principle only. First, we observe that if the upper estimate of (1.2) holds at some time, then so does it after that time with exponentially decaying constant $C_{t}$.

Proposition 2.1. Suppose $p\left(t_{0}, x, y\right) \leq C_{t_{0}} \varphi_{D}(x) \varphi_{D}(y)$ for all $x, y \in D$ with some $t_{0}>0$. If $t \geq t_{0}$, then

$$
p(t, x, y) \leq C_{t_{0}} e^{-\lambda_{D}\left(t-t_{0}\right)} \varphi_{D}(x) \varphi_{D}(y) \quad \text { for all } x, y \in D .
$$

In other words, $p(t, x, y) \leq C_{t} \varphi_{D}(x) \varphi_{D}(y)$ holds with $C_{t} \leq C_{t_{0}} e^{-\lambda_{D}\left(t-t_{0}\right)}$ for $t \geq t_{0}$.

Proof. Fix $y \in D$. It is easy to see that $u(t, x)=C_{t_{0}} e^{-\lambda_{D}\left(t-t_{0}\right)} \varphi_{D}(x) \varphi_{D}(y)$ is a caloric function with vanishing lateral boundary values. By assumption, $p\left(t_{0}, x, y\right) \leq u\left(t_{0}, x\right)$ for $x \in D$. Hence the comparison principle on $\left(t_{0}, \infty\right) \times D$ yields the required inequality.

Second, we observe that the upper estimate of IU automatically implies the lower estimate.

Proposition 2.2. Suppose, for every $t>0$, there exists $C_{t}>0$ such that

$$
p(t, x, y) \leq C_{t} \varphi_{D}(x) \varphi_{D}(y) \quad \text { for all } x, y \in D .
$$

Then, for every $t>0$, there exists $C_{t}^{\prime}>0$ such that

$$
p(t, x, y) \geq C_{t}^{\prime} \varphi_{D}(x) \varphi_{D}(y) \quad \text { for all } x, y \in D .
$$

Proof. Represent a caloric function $e^{-\lambda_{D} t} \varphi_{D}(x)$ by an integral,

$$
e^{-\lambda_{D} t} \varphi_{D}(x)=\int_{D} p(t, x, y) \varphi_{D}(y) d y .
$$

Let $K=K(t)$ be a compact subset of $D$ to be determined later. Split the integral domain $D$ into $D \backslash K$ and $K$ and then apply the upper estimate to the first integral. We have

$$
e^{-\lambda_{D} t} \varphi_{D}(x) \leq \int_{D \backslash K} C_{t} \varphi_{D}(x) \varphi_{D}(y)^{2} d y+\int_{K} p(t, x, y) \varphi_{D}(y) d y .
$$

Since $\varphi_{D} \in L^{2}(D)$, we can choose $K=K(t)$ so large that the first integral is less than $2^{-1} e^{-\lambda_{D} t} \varphi_{D}(x)$. Hence we obtain

$$
e^{-\lambda_{D} t} \varphi_{D}(x) \leq 2 \int_{K} p(t, x, y) \varphi_{D}(y) d y .
$$

Observe that $\alpha=\inf \left\{\frac{p(t, z, w)}{\varphi_{D}(z) \varphi_{D}(w)}: z, w \in K\right\}>0$. 
By the semigroup property we have

$$
\begin{aligned}
& p(3 t, x, y)=\int_{D}\left(\int_{D} p(t, x, z) p(t, z, w) d z\right) p(t, w, y) d w \\
& \geq \int_{K} \int_{K} p(t, x, z) p(t, z, w) p(t, w, y) d z d w \\
& \geq \alpha \int_{K} \int_{K} p(t, x, z) \varphi_{D}(z) \varphi_{D}(w) p(t, w, y) d z d w \geq \frac{\alpha}{4} e^{-2 \lambda_{D} t} \varphi_{D}(x) \varphi_{D}(y),
\end{aligned}
$$

where we have used the symmetry of $p(t, w, y)$ and (2.1) twice in the last inequality. Hence changing $3 t$ by $t$ gives the required lower estimate.

Third, we prove that IU implies the Cranston-McConnell inequality. This fact is well known to probabilists. However we have not been able to find a good reference in the literature except for Theorem 2.8 in [24], which deals with a nonsymmetric case.

Proposition 2.3. If IU holds, then there exists a positive constant A such that

$$
\sup _{x \in D} \frac{1}{u(x)} \int_{D} G(x, y) u(y) d y \leq A
$$

whenever $u$ is a positive superharmonic function on $D$.

Proof. In view of Fubini's theorem and (1.26), we have

$$
\int_{D} G(x, y) u(y) d y=\int_{0}^{1} d t \int_{D} p(t, x, y) u(y) d y+\int_{1}^{\infty} d t \int_{D} p(t, x, y) u(y) d y .
$$

Let us estimate the two integrals in the right-hand side separately. Regard $u(x)$ as a stationary supercaloric function. By the comparison principle

$$
u(x) \geq \int_{D} p(t, x, y) u(y) d y,
$$

since the right-hand side is a caloric function with vanishing lateral boundary values. Hence

$$
\int_{0}^{1} d t \int_{D} p(t, x, y) u(y) d y \leq \int_{0}^{1} u(x) d t=u(x) .
$$

Observe from (2.2) with $t=1$ and the lower estimate of IU that

$$
u(x) \geq \int_{D} p(1, x, y) u(y) d y \geq C_{1}^{\prime} \int_{D} \varphi_{D}(x) \varphi_{D}(y) u(y) d y .
$$

Hence the upper estimate of IU with control of $C_{t}$ (Proposition 2.1) gives

$$
\int_{1}^{\infty} d t \int_{D} p(t, x, y) u(y) d y \leq \int_{1}^{\infty} d t \int_{D} C_{1} e^{-\lambda_{D}(t-1)} \varphi_{D}(x) \varphi_{D}(y) u(y) d y \leq \frac{C_{1}}{\lambda_{D} C_{1}^{\prime}} u(x) .
$$

Thus we obtain the required Cranston-McConnell inequality.

Finally, we observe that the GBHP can be characterized by the estimate (1.12) of the Green function. 
Proposition 2.4. The GBHP holds if and only if (1.12) holds for every disjoint compact sets $K$ and $F$ intersecting $D$ such that $x_{0} \notin K \cup F$.

Proof. First we prove the 'only if' part. Let $K$ and $F$ be disjoint compact sets intersecting $D$ such that $x_{0} \notin K \cup F$. Let $R=\operatorname{dist}\left(K, F \cup\left\{x_{0}\right\}\right)>0$ and put $V=\left\{x \in \mathbb{R}^{n}: \operatorname{dist}(x, K)<R / 2\right\}$. Then $V$ is a bounded open set containing $K$ such that $\bar{V} \cap\left(F \cup\left\{x_{0}\right\}\right)=\emptyset$. Apply (1.11) to $u=G(\cdot, y) / g(y)$ with $y \in F \cap D$ and $v=g$. If $x, x_{1} \in K \cap D$, then

$$
A \geq \frac{u(x) / u\left(x_{1}\right)}{v(x) / v\left(x_{1}\right)}=\frac{G(x, y) / G\left(x_{1}, y\right)}{g(x) / G\left(x_{1}, x_{0}\right)} \approx \frac{G(x, y)}{g(x) g(y)},
$$

since $g\left(x_{1}\right)=G\left(x_{1}, x_{0}\right) \approx 1$ and $G\left(x_{1}, y\right) \approx G\left(x_{0}, y\right)=g(y)$ by the usual Harnack principle and the symmetry of the Green function. Replacing the roles of $u$ and $v$, we obtain (1.12).

Next we prove the 'if' part. Let $(V, K)$ be a pair consisting of a bounded open set $V \subset \mathbb{R}^{n}$ and a compact set $K \subset \mathbb{R}^{n}$ such that $K \subset V, K \cap D \neq \emptyset$ and $K \cap \partial D \neq \emptyset$. Suppose $u$ and $v$ are positive superharmonic functions on $D$ such that

(i) $u$ and $v$ are bounded, positive and harmonic in $V \cap D$,

(ii) $u$ and $v$ vanish on $V \cap \partial D$ outside a polar set.

Let us prove (1.11). Without loss of generality we may assume that $x_{0} \in D \backslash \bar{V}$. Let $W$ be an open set such that $K \subset W \subset \bar{W} \subset V$ and let $F=\partial W$. Observe that ${ }^{D} \widehat{\mathbf{R}}_{u}^{F \cap D}$ and ${ }^{D} \widehat{\mathbf{R}}_{v}^{F \cap D}$ are Green potentials which coincide with $u$ and $v$ in $W \cap D$, respectively. By the Riesz decomposition theorem we find measures $\mu$ and $\nu$, concentrated on $F \cap D$, such that

$$
u=\int_{F \cap D} G(\cdot, z) d \mu(z), \quad v=\int_{F \cap D} G(\cdot, z) d \nu(z) \quad \text { on } W \cap D .
$$

See Figure 2.

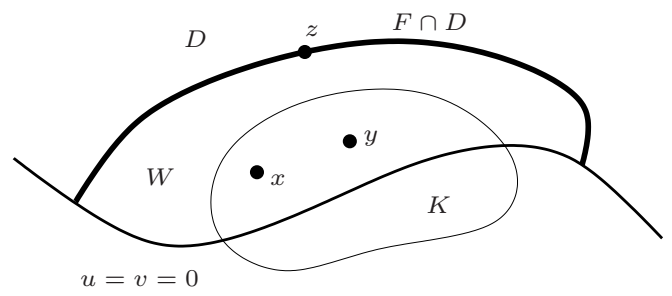

Figure 2. Green kernel estimate $\Longrightarrow$ GBHP.

Evaluating $u$ at $x \in K \cap D$, we obtain from (1.12) with $z$ in place of $y$ that $u(x) \approx g(x) \int_{F \cap D} g(z) d \mu(z)$. We have similar estimates for $u(y), v(x)$ and $v(y)$. Hence

$$
\frac{u(x) / u(y)}{v(x) / v(y)} \approx \frac{\left\{g(x) \int_{F \cap D} g(z) d \mu(z)\right\} /\left\{g(y) \int_{F \cap D} g(z) d \mu(z)\right\}}{\left\{g(x) \int_{F \cap D} g(z) d \nu(z)\right\} /\left\{g(y) \int_{F \cap D} g(z) d \nu(z)\right\}}=1 .
$$

Thus (1.11) follows. 


\section{First eigenvalue, capacitary width and related widths. Proof of Theorem 1.5}

\subsection{Proof of Theorem 1.5}

Capacitary width gives a two-sided estimate of the supremum of the Green potential of constant density. In this section we let

$$
v(x)=\int_{D} G(x, y) d y
$$

The following lemma is known in the case $\mathcal{L}=\Delta$. But, for completeness, we shall provide a proof in the appendix.

Lemma $\mathbf{D}$ (Theorem 1 in [1]). There is a constant $A>1$ depending only on $c_{\mathcal{L}}$, $\eta$ and $n$ such that

$$
A^{-1} w_{\eta}(D)^{2} \leq\|v\|_{\infty} \leq A w_{\eta}(D)^{2}
$$

Let us consider a parabolic counterpart. For simplicity let us write $\pi(t)=$ $\sup _{x \in D} P(t, x, D)$. We shall show several relationships among $\pi(t),\|v\|_{\infty}$, and the first eigenvalue $\lambda_{D}$. The following theorem, together with Lemma $\mathrm{D}$, readily yields Theorem 1.5.

Theorem 3.1. The following conditions are equivalent:

(i) $\lambda_{D}>0$.

(ii) $w_{\eta}(D)<\infty$.

(iii) $\|v\|_{\infty}<\infty$.

(iv) $\pi(t)<1$ for some $t>0$.

(v) $\pi(t) \leq A \exp (-\alpha t)$ for all $t>0$ with some $A, \alpha>0$.

Moreover,

$$
\frac{A^{-1}}{w_{\eta}(D)^{2}} \leq \frac{1}{\|v\|_{\infty}} \leq \lambda_{D} \leq \frac{A}{\|v\|_{\infty}} \leq \frac{A^{2}}{w_{\eta}(D)^{2}}
$$

where $A>1$ depends only on $c_{\mathcal{L}}, \eta$ and $n$.

See [13] and [14] for the second and third inequalities of (3.1). Our argument is inspired by Souplet [37]. First we give a lower estimate of $\pi(t)$.

Lemma 3.2. We have

$$
\exp \left(-\lambda_{D} t\right) \leq \pi(t) \quad \text { for all } t>0
$$

Proof. Let $\beta>\lambda_{D}$. Then we find $\varphi \in C_{0}^{\infty}(D)$ such that $Q_{D}[\varphi] /\|\varphi\|_{2}^{2} \leq \beta$. We may assume that $\varphi \geq 0$. Take a relatively compact subdomain $\Omega$ such that 
$\operatorname{supp} \varphi \subset \Omega \Subset D$. Let $\lambda_{\Omega}$ and $\varphi_{\Omega} \geq 0$ be the first eigenvalue and its eigenfunction with $\left\|\varphi_{\Omega}\right\|_{2}=1$ for $\Omega$, respectively. By definition

$$
\lambda_{\Omega}=\inf \left\{\frac{Q_{\Omega}[\psi]}{\|\psi\|_{2}^{2}}: \psi \in C_{0}^{\infty}(\Omega)\right\} \leq \frac{Q_{D}[\varphi]}{\|\varphi\|_{2}^{2}} \leq \beta
$$

Since $u(t, x)=\exp \left(-\lambda_{\Omega} t\right) \varphi_{\Omega}(x)$ is a caloric function in $(0, \infty) \times \Omega$ such that $u(0, x)=\varphi_{\Omega}(x)$ and $u(t, x)=0$ on $(0, \infty) \times \partial \Omega$, it follows from the comparison principle that

$$
\exp \left(-\lambda_{\Omega} t\right) \varphi_{\Omega}(x) \leq \int_{\Omega} p(t, x, y) \varphi_{\Omega}(y) d y \leq\left\|\varphi_{\Omega}\right\|_{\infty} P(t, x, D) \leq\left\|\varphi_{\Omega}\right\|_{\infty} \pi(t)
$$

on $(0, \infty) \times \Omega$. Now, taking the supremum for $x \in \Omega$, and then dividing by $0<\left\|\varphi_{\Omega}\right\|_{\infty}<\infty$, we obtain

$$
\exp (-\beta t) \leq \exp \left(-\lambda_{\Omega} t\right) \leq \pi(t)
$$

Since $\beta>\lambda_{D}$ is arbitrary, we have the required inequality.

Next we give an upper estimate of $\pi(t)$ in terms of $\|v\|_{\infty}$, which readily yields Proposition 1.8.

Lemma 3.3. Let $C>1$. If $\|v\|_{\infty}<\infty$, then

$$
\pi(t) \leq \frac{C}{C-1} \exp \left(-\frac{t}{C\|v\|_{\infty}}\right) \text { for all } t>0 .
$$

Proof. Let $\alpha=1 /\left(C\|v\|_{\infty}\right)$ and let $w(t, x)=\left(v(x)+(C-1)\|v\|_{\infty}\right) e^{-\alpha t}$. Since $v$ enjoys the Poisson equation $-\mathcal{L} v=1$ in $D$, it follows that

$$
\begin{aligned}
\left(\mathcal{L}-\frac{\partial}{\partial t}\right) w & =\mathcal{L} v \cdot e^{-\alpha t}+\alpha\left(v+(C-1)\|v\|_{\infty}\right) e^{-\alpha t}=\left(-1+\frac{v+(C-1)\|v\|_{\infty}}{C\|v\|_{\infty}}\right) e^{-\alpha t} \\
& \leq\left(-1+\frac{\|v\|_{\infty}+(C-1)\|v\|_{\infty}}{C\|v\|_{\infty}}\right) e^{-\alpha t}=0 .
\end{aligned}
$$

Hence $w$ is supercaloric. By the comparison principle,

$$
(C-1)\|v\|_{\infty} P(t, x, D) \leq w(t, x)=\left(v(x)+(C-1)\|v\|_{\infty}\right) e^{-\alpha t} \leq C\|v\|_{\infty} e^{-\alpha t} .
$$

Dividing the inequality by $0<\|v\|_{\infty}<\infty$, and taking the supremum for $x \in D$, we obtain the lemma.

Proof of Proposition 1.8. Combine Lemma D and Lemma 3.3 with $C>1$ fixed.

Let us give another upper estimate of $\pi(t)$ in terms of $\lambda_{D}$. The following lemma is a preliminary $L^{2}$-estimate, which may be well known. However, we give a proof for completeness. 
Lemma 3.4. For $\varphi \in L^{2}(D)$ let $u(t, x)=\int_{D} p(t, x, y) \varphi(y) d y$. Then

$$
\|u(t, \cdot)\|_{2} \leq e^{-\lambda_{D} t}\|\varphi\|_{2} \text {. }
$$

Proof. First assume that $D$ is bounded. Then the spectrum of $-\mathcal{L}$ is given by eigenvalues $\lambda_{1}<\lambda_{2} \leq \ldots$ with corresponding orthonormal set of eigenfunctions $\left\{\varphi_{1}, \varphi_{2}, \ldots\right\}$; the heat kernel is expanded as $p(t, x, y)=\sum_{j=1}^{\infty} e^{-\lambda_{j} t} \varphi_{j}(x) \varphi_{j}(y)$ in $L^{2}$. Let $\varphi \in L^{2}(D)$. Writing $\varphi=\sum_{i=1}^{\infty} c_{i} \varphi_{i}$ with $\|\varphi\|_{2}^{2}=\sum_{i=1}^{\infty} c_{i}^{2}$, we obtain

$$
u(t, x)=\int_{D} \sum_{i, j} e^{-\lambda_{j} t} \varphi_{j}(x) \varphi_{j}(y) c_{i} \varphi_{i}(y) d y=\sum_{j=1}^{\infty} e^{-\lambda_{j} t} c_{j} \varphi_{j}(x),
$$

so that

$$
\begin{aligned}
\|u(t, \cdot)\|_{2}^{2} & =\int_{D} \sum_{i, j} e^{-\lambda_{i} t} c_{i} \varphi_{i}(x) e^{-\lambda_{j} t} c_{j} \varphi_{j}(x) d x=\sum_{j=1}^{\infty} e^{-2 \lambda_{j} t} c_{j}^{2} \\
& \leq e^{-2 \lambda_{1} t} \sum_{j=1}^{\infty} c_{j}^{2}=e^{-2 \lambda_{1} t}\|\varphi\|_{2}^{2} .
\end{aligned}
$$

Taking the square root, we obtain the required estimate, as $\lambda_{1}=\lambda_{D}$. In case $D$ is unbounded, we observe that $\lambda_{D} \leq \lambda_{D \cap B(0, R)}$ and $p_{D \cap B(0, R)}(t, x, y) \uparrow p_{D}(t, x, y)$ as $R \rightarrow \infty$. Let $\varphi \in L^{2}(D)$. Without loss of generality we may assume that $\varphi \geq 0$. Then

$$
u_{R}(t, x)=\int_{D \cap B(0, R)} p_{D \cap B(0, R)}(t, x, y) \varphi(y) d y \uparrow u(t, x) .
$$

The first case gives $\left\|u_{R}(t, \cdot)\right\|_{2}^{2} \leq e^{-2 \lambda_{D \cap B(0, R)} t}\|\varphi\|_{2}^{2} \leq e^{-2 \lambda_{D} t}\|\varphi\|_{2}^{2}$, so that the monotone convergence theorem completes the proof.

Lemma 3.5. Let $C>1$. There exists a constant $M(C)>1$ depending only on $C$, $c_{\mathcal{L}}$ and $n$ such that

$$
\pi(t) \leq M(C) \exp \left(-\frac{\lambda_{D} t}{C}\right) \text { for all } t>0 .
$$

Moreover, if $\lambda_{D}>0$, then $\|v\|_{\infty} \leq A / \lambda_{D}$.

Proof. Let $x \in D$ fixed. For $R>0$ to be determined later, we put $\Omega=D \cap B(x, R)$. Let us estimate $P(t, x, D)=\int_{D} p(t, x, y) d y$ by decomposing $D$ into $\Omega$ and $D \backslash \Omega$. Let $0<\varepsilon<1-1 / C$. By the semigroup property, Fubini's theorem, Lemma 3.4 with $\varphi=\chi_{\Omega}$, and (1.25) we have

$$
\begin{aligned}
\int_{\Omega} p(t, x, y) d y & =\int_{D} p(\varepsilon t, x, z)\left(\int_{\Omega} p((1-\varepsilon) t, z, y) d y\right) d z \\
& \leq\left\{\int_{D} p(\varepsilon t, x, z)^{2} d z\right\}^{1 / 2}\left\{\int_{D}\left(\int_{\Omega} p((1-\varepsilon) t, z, y) d y\right)^{2} d z\right\}^{1 / 2} \\
& \leq \frac{A}{(\varepsilon t)^{n / 2}}\left\{\int_{\mathbb{R}^{n}} \exp \left(-A^{\prime} \frac{|x-z|^{2}}{\varepsilon t}\right) d z\right\}^{1 / 2} e^{-\lambda_{D}(1-\varepsilon) t}|\Omega|^{1 / 2} \\
& \leq \frac{A}{(\varepsilon t)^{n / 4}} \exp \left(-\lambda_{D}(1-\varepsilon) t\right) R^{n / 2}
\end{aligned}
$$


On the other hand, (1.25) and the change of the variable $x-y=\sqrt{t} z$ give

$$
\begin{aligned}
& \int_{D \backslash \Omega} p(t, x, y) d y \leq \frac{A}{t^{n / 2}} \int_{|y-x| \geq R} \exp \left(-\frac{A^{\prime}|x-y|^{2}}{t}\right) d y \\
& \quad=A \int_{|z| \geq R / \sqrt{t}} \exp \left(-A^{\prime}|z|^{2}\right) d z \\
& \quad \leq A \exp \left(-\frac{A^{\prime} R^{2}}{2 t}\right) \int_{|z| \geq R / \sqrt{t}} \exp \left(-\frac{A^{\prime}|z|^{2}}{2}\right) d z \leq A \exp \left(-\frac{A^{\prime} R^{2}}{2 t}\right)
\end{aligned}
$$

since $\exp \left(-A^{\prime}|z|^{2} / 2\right)$ is integrable over $\mathbb{R}^{n}$. Letting $R=t \sqrt{2 \lambda_{D} / A^{\prime}}$, we obtain $P(t, x, D) \leq A \varepsilon^{-n / 4} \lambda_{D}^{n / 4} t^{n / 4} \exp \left(-\lambda_{D}(1-\varepsilon) t\right)+A \exp \left(-\lambda_{D} t\right) \leq A \exp \left(-\frac{\lambda_{D} t}{C}\right)$,

since $1-\varepsilon>1 / C$. Taking the supremum for $x \in D$, we obtain the first assertion of the theorem with $M(C)=A$. Suppose $\lambda_{D}>0$. By (1.26) and Fubini's theorem we have

$$
\int_{D} G(x, y) d y=\int_{0}^{\infty} P(t, x, D) d t \leq \int_{0}^{\infty} \pi(t) d t \leq A \int_{0}^{\infty} \exp \left(-\frac{\lambda_{D} t}{C}\right) d t=\frac{A C}{\lambda_{D}} .
$$

Taking the supremum for $x \in D$, we obtain the second assertion.

Proof of Theorem 3.1. We have the following implications, which complete the first assertion of the theorem:

(ii) $\Longleftrightarrow$ (iii) by Lemma D.

(i) $\Longrightarrow$ (iii), (v) by Lemma 3.5.

(v) $\Longrightarrow$ (iv) trivial.

(iv) $\Longrightarrow$ (i) by Lemma 3.2 .

(iii) $\Longrightarrow$ (v) by Lemma 3.3 .

We have the comparison $\|v\|_{\infty} \approx w_{\eta}(D)^{2}$ by Lemma D. If $\|v\|_{\infty}<\infty$, then Lemmas 3.2 and 3.3 give

$$
\exp \left(-\lambda_{D} t\right) \leq \pi(t) \leq \frac{C}{C-1} \exp \left(-\frac{t}{C\|v\|_{\infty}}\right) \text { for all } t>0,
$$

whenever $C>1$. Hence $\lambda_{D} \geq 1 /\left(C\|v\|_{\infty}\right)$ for every $C>1$, and so $\lambda_{D} \geq 1 /\|v\|_{\infty}$. Conversely, if $\lambda_{D}>0$, then Lemma 3.5 gives $\|v\|_{\infty} \leq A / \lambda_{D}$. Thus we have

$$
\frac{1}{\|v\|_{\infty}} \leq \lambda_{D} \leq \frac{A}{\|v\|_{\infty}}
$$

which, together with Lemma D, yields (3.1) and, in particular, (1.5).

Remark 3.6. Let $\mathcal{L}=\Delta$ and let $D$ be the infinite strip $\{(x, y): x \in \mathbb{R},|y|<\pi / 2\}$ in $\mathbb{R}^{2}$. Then it is easy to see that $\lambda_{D}=1$ and $\varphi_{D}(x, y)=\cos y$. So, $\varphi_{D} \notin$ $L^{2}(D)$. Obviously, $w_{\eta}(D)=A \pi$ with $A>1$ depending only on $\eta$; moreover, $w_{\eta}(D \backslash \bar{B}(0, R))=A \pi$ for every $R>0$. Hence (1.6) does not hold. 


\subsection{Volumetric width and inradius}

Replacing capacity by volume, we obtain another width related to capacitary width.

Definition 3.7. Let $0<\eta<1$. For an open set $D$ we define the volumetric width $v_{\eta}(D)$ by

$$
v_{\eta}(D)=\inf \left\{r>0: \frac{|B(x, r) \backslash D|}{|B(x, r)|} \geq \eta \quad \text { for all } x \in D\right\} .
$$

We see that volumetric width is essentially larger than capacitary width. Let us begin with a comparison between capacity and volume.

Lemma 3.8. Let $\tau>1$. Then there is a positive constant $A_{4}$ depending only on $\tau$ and $n$ such that

$$
\frac{|E|}{|B(x, r)|} \leq A_{4} \frac{\operatorname{Cap}_{B(x, \tau r)}(E)}{\operatorname{Cap}_{B(x, \tau r)}(B(x, r))}
$$

for every Borel set $E \subset B(x, r)$.

Proof. Let us use the characterization (1.3) of capacity. In this proof, the Green function is for the Laplacian. Let $K \subset E$ be an arbitrary compact set and let $\mu$ be the restriction of Lebesgue measure over $K$. Since

$$
\int_{B(x, \tau r)} G_{B(x, \tau r)}(y, z) d \mu(z) \leq \int_{B(x, \tau r)} G_{B(x, \tau r)}(y, z) d z \leq A r^{2} \text { for all } y \in B(x, \tau r),
$$

where $A$ depends only on $\tau$ and $n$, it follows from (1.3) that $\operatorname{Cap}_{B(x, \tau r)}(E) \geq$ $A^{-1} r^{-2}|K|$, so that $\operatorname{Cap}_{B(x, \tau r)}(E) \geq A^{-1} r^{-2}|E|$, as $K \subset E$ is arbitrary. Dividing the inequality by $\operatorname{Cap}_{B(x, \tau r)}(B(x, r))=A r^{n-2}$, we obtain the lemma.

Lemma 3.9. There is a positive constant $A$ depending only on $\eta$ and $n$ such that

$$
w_{\eta}(D) \leq A v_{\eta}(D) \quad \text { for every open set } D .
$$

Proof. Let $D$ be an open set with $v_{\eta}(D)<\infty$. By definition we find $r \geq v_{\eta}(D)$ arbitrarily close to $v_{\eta}(D)$ such that $|B(x, r) \backslash D| /|B(x, r)| \geq \eta$ for all $x \in D$. By Lemma 3.8 with $\tau=2$ we have

$$
\eta \leq \frac{|B(x, r) \backslash D|}{|B(x, r)|} \leq A_{4} \frac{\operatorname{Cap}_{B(x, 2 r)}(B(x, r) \backslash D)}{\operatorname{Cap}_{B(x, 2 r)}(B(x, r))} \text { for all } x \in D .
$$

Hence $w_{\eta / A_{4}}(D) \leq r$, and so $w_{\eta / A_{4}}(D) \leq v_{\eta}(D)$ by the arbitrariness of $r$. Since, by Lemma $12.8, w_{\eta}(D) \approx w_{\eta^{\prime}}(D)$ for $0<\eta, \eta^{\prime}<1$, we obtain the required inequality.

Volume and volumetric width have the following relationship.

Lemma 3.10. There is a positive constant $A$ depending only on $\eta$ and $n$ such that

$$
v_{\eta}(D) \leq A|D|^{1 / n} \quad \text { for every open set } D .
$$


Proof. We may assume that $|D|<\infty$. Let $R>0$ be such that $(1-\eta)|B(0, R)|=$ $|D|$, i.e., $b_{n}(1-\eta) R^{n}=|D|$ with $b_{n}$ being the volume of a unit ball. Then, for every $x \in D$,

$$
(1-\eta)^{-1}|D|=|B(x, R) \cap D|+|B(x, R) \backslash D| \leq|D|+|B(x, R) \backslash D|,
$$

so that

$$
\frac{\eta}{1-\eta}=\frac{1}{1-\eta}-1 \leq \frac{|B(x, R) \backslash D|}{|D|}=\frac{|B(x, R) \backslash D|}{(1-\eta)|B(x, R)|} .
$$

Hence $v_{\eta}(D) \leq R=\left(\frac{|D|}{b_{n}(1-\eta)}\right)^{1 / n}$, as required.

In view of (3.1), Lemmas 3.9 and 3.10 we have the following lower bound of the first eigenvalue.

Corollary 3.11. There are positive constants $A$ and $A^{\prime}$ depending only on $c_{\mathcal{L}}, \eta$ and $n$ such that

$$
\lambda_{D} \geq \frac{A}{v_{\eta}(D)^{2}} \geq \frac{A^{\prime}}{|D|^{2 / n}} .
$$

Remark 3.12. The first inequality of Corollary 3.11 was first proved by Lieb [25]; the second is the classical Faber-Krahn inequality modulo sharp constant.

Another related quantity is the inradius:

$$
\operatorname{Inr}(D)=\sup \left\{\delta_{D}(x): x \in D\right\} .
$$

In other words, $\operatorname{Inr}(D)$ is the supremum of radii of balls included in $D$. Obviously, $\operatorname{Inr}(D) \leq v_{\eta}(D)$ and $\operatorname{Inr}(D) \leq w_{\eta}(D)$. The opposite inequality (up to a multiplicative constant) of the last inequality holds if $D$ satisfies the CDC up to $\infty$. To see this, recall the following estimate.

Lemma 3.13 (Proposition 1 in [1]). Suppose D satisfies the CDC up to $r_{0}$. Then there is a positive constant $A$ such that

$$
w_{\eta}\left(\left\{x \in D: \delta_{D}(x)<r\right\}\right) \leq A r \quad \text { for } 0<r<r_{0} .
$$

In particular, if $D$ satisfies the $C D C$ up to $\infty$, then $\operatorname{Inr}(D) \approx w_{\eta}(D)$.

Corollary 3.14. If $D$ satisfies the CDC up to $\infty$, then $\lambda_{D} \approx 1 / \operatorname{Inr}(D)^{2}$.

Remark 3.15. Corollary 3.14 was proved by Ancona [6] by establishing the Hardy inequality. Maz'ya-Shubin [26] obtained the same estimate by using $r_{D, \gamma}$. See Remark 1.6.

Remark 3.16. We say that $D$ is a John domain if there exist a positive constant $c_{J}$ and a point $x_{0} \in D$ for which each $x \in D$ can be connected to $x_{0}$ by a rectifiable curve $\gamma \subset D$ such that

$$
\delta_{D}(y) \geq c_{J} \ell(\gamma(x, y)) \quad \text { for all } y \in \gamma,
$$

where $\gamma(x, y)$ is the subarc of $\gamma$ from $x$ to $y$ and $\ell(\gamma(x, y))$ is the length of $\gamma(x, y)$. 
Then (3.2) holds for a John domain $D$ without the CDC. In fact, let $U=\{x \in D$ : $\left.\delta_{D}(x)<r\right\}$ with $\left(1+2 / c_{J}\right) r<\delta_{D}\left(x_{0}\right)$. If $x \in U$, then $\left|x_{0}-x\right| \geq \delta_{D}\left(x_{0}\right)-r>$ $2 r / c_{J}$. Let $\gamma$ be the curve connecting $x$ and $x_{0}$ as above. We find a point $y \in \gamma$ such that $\ell(\gamma(x, y))=2 r / c_{J}$. Then $\delta_{D}(y) \geq 2 r$ and $|x-y| \leq 2 r / c_{J}$, so that $B(y, r) \subset B\left(x,\left(1+2 / c_{J}\right) r\right) \backslash U$, and hence

$$
\frac{\left|B\left(x,\left(1+2 / c_{J}\right) r\right) \backslash U\right|}{\left|B\left(x,\left(1+2 / c_{J}\right) r\right)\right|} \geq \frac{|B(y, r)|}{\left|B\left(x,\left(1+2 / c_{J}\right) r\right)\right|}=\frac{1}{\left(1+2 / c_{J}\right)^{n}} .
$$

Since $x \in U$ is arbitrary, it follows that $v_{\eta}(U) \leq\left(1+2 / c_{J}\right) r$ with $\eta=\left(1+2 / c_{J}\right)^{-n}$. Hence $w_{\eta}(U) \leq A r$ by Lemma 3.9. This is the key observation to dispense with barrier conditions from the boundary Harnack principle.

Finally, we prove that (1.6) is not preserved by taking the union of two domains.

Proposition 3.17. There are two domains $D_{0}$ and $D_{1}$ such that $\lim _{R \rightarrow \infty} w_{\eta}\left(D_{0} \backslash\right.$ $\bar{B}(0, R))=\lim _{R \rightarrow \infty} w_{\eta}\left(D_{1} \backslash \bar{B}(0, R)\right)=0$ and yet $D_{0} \cup D_{1}=\mathbb{R}^{n}$.

Proof. For simplicity we assume that $n=2$ and use complex notation. The $n \geq 3$ case is similar and is left to the reader. We place countably many small closed disks so that their complement satisfies the required properties. See Figure 3.

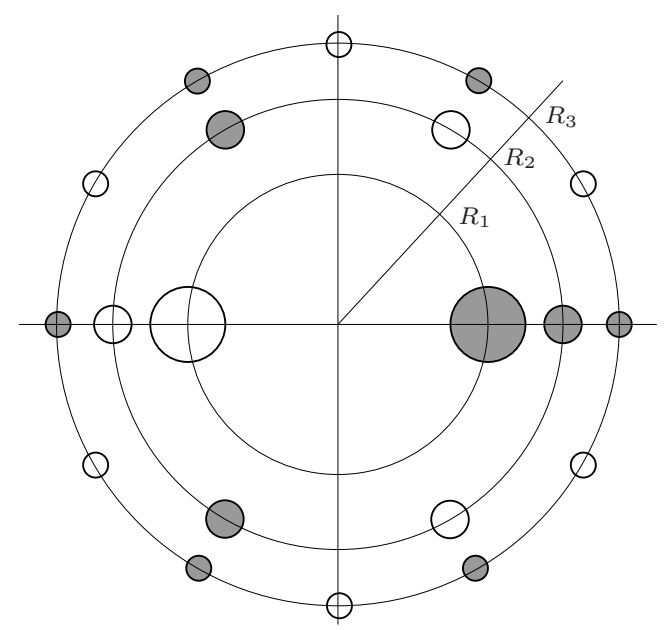

FiguRE 3. Domains outside closed disks; $D_{0}=\mathbb{R}^{2} \backslash \bigcup_{k=1}^{\infty} E_{k}^{0}$ with $E_{k}^{0}$ being the union of shaded disks. $R_{1}=1, R_{2}=3 / 2, R_{3}=11 / 6, \ldots$

For a positive integer $k$ we let

$$
R_{k}=\frac{1}{1}+\cdots+\frac{1}{k} \quad \text { and } \quad k R_{k} \leq N_{k}<k R_{k}+1
$$

We place $2 N_{k}$ many points $\left\{R_{k} \exp \left(i \pi j / N_{k}\right): 0 \leq j \leq 2 N_{k}-1\right\}$ uniformly on the circle $\left\{|z|=R_{k}\right\}$. Then the distance $d_{k}$ between two consecutive points satisfies

$$
\frac{1}{k}<2 \frac{R_{k}}{N_{k}} \leq d_{k}=2 R_{k} \sin \frac{\pi}{2 N_{k}} \leq \pi \frac{R_{k}}{N_{k}} \leq \frac{\pi}{k} .
$$


Hence

$$
E_{k}=\bigcup_{j=0}^{2 N_{k}-1} \bar{B}\left(R_{k} \exp \left(\frac{i \pi j}{N_{k}}\right), \frac{1}{4 k}\right)
$$

is the union of $2 N_{k}$ many disjoint closed disks. In particular, the even union $E_{k}^{0}$ and the odd union $E_{k}^{1}$ are disjoint, i.e., $E_{k}^{0} \cap E_{k}^{1}=\emptyset$ with

$$
E_{k}^{0}=\bigcup_{j=0}^{N_{k}-1} \bar{B}\left(R_{k} \exp \left(\frac{i \pi 2 j}{N_{k}}\right), \frac{1}{4 k}\right) \quad \text { and } \quad E_{k}^{1}=\bigcup_{j=0}^{N_{k}-1} \bar{B}\left(R_{k} \exp \left(\frac{i \pi(2 j+1)}{N_{k}}\right), \frac{1}{4 k}\right) .
$$

Moreover,

since

$$
R_{k}+\frac{1}{4 k}<R_{k+1}-\frac{1}{4(k+1)}
$$

$$
R_{k+1}-R_{k}=\frac{1}{k+1}>\frac{1}{2 k}>\frac{1}{4 k}+\frac{1}{4(k+1)} .
$$

This means that $\left\{E_{k}\right\}_{k=1}^{\infty}$ is pairwise disjoint, so that

$$
D_{0} \cup D_{1}=\mathbb{R}^{2} \quad \text { with } D_{0}=\mathbb{R}^{2} \backslash \bigcup_{k=1}^{\infty} E_{k}^{0} \text { and } D_{1}=\mathbb{R}^{2} \backslash \bigcup_{k=1}^{\infty} E_{k}^{1}
$$

It is easy to see from construction that $\lim _{R \rightarrow \infty} v_{\eta}\left(D_{j} \backslash \bar{B}(0, R)\right)=0$ for $j=0,1$, and hence $\lim _{R \rightarrow \infty} w_{\eta}\left(D_{j} \backslash \bar{B}(0, R)\right)=0$ by Lemma 3.9 .

\section{Parabolic box argument}

The Green function $g(x)=G\left(\cdot, x_{0}\right)$ can be regarded as a time-independent caloric function on $(0, \infty) \times\left(D \backslash\left\{x_{0}\right\}\right)$. In this section we shall show a decay estimate of $P(t, x, D)$ as $x$ approaches the boundary $\partial D$ in terms of $g(x)$. We shall invoke a parabolic box argument, a parabolic counterpart of the box argument for the boundary Harnack principle. See Section 6. See also [11], [5] and references therein. The decomposition of the domain $D$ with respect to the level surfaces of $g$ plays an important role. First we give a general framework.

Lemma 4.1. Let $\left\{\alpha_{j}\right\}_{j=1}^{\infty}$ be a decreasing sequence converging to 0 and let $\left\{t_{j}\right\}_{j=0}^{\infty}$ be an increasing sequence converging to $t_{\infty}<\infty$ with $t_{0}=0$. Put $D_{j}=\{x \in D$ : $\left.g(x)<\alpha_{j}\right\}$. If $t \geq t_{\infty}$, then $P(t, x, D) \leq A^{*} g(x)$ for $x \in D$, where

$$
A^{*}=\frac{1}{\alpha_{1}}+\sum_{j=1}^{\infty} \frac{A_{0}}{\alpha_{j+1}} \exp \left(-\frac{A_{1}\left(t_{j}-t_{j-1}\right)}{w_{\eta}\left(D_{j}\right)^{2}}\right) .
$$

Proof. Let $E_{j}=\left\{x \in D: \alpha_{j+1} \leq g(x)<\alpha_{j}\right\}, \widetilde{D}_{j}=\left(t_{j-1}, \infty\right) \times D_{j}$ and $\widetilde{E}_{j}=$ $\left(t_{j}, \infty\right) \times E_{j}$. Put

$$
q_{j}=\sup _{(t, x) \in \widetilde{E}_{j}} \frac{P(t, x, D)}{g(x)} .
$$

It is sufficient to show that $\sup _{j \geq 1} q_{j} \leq A^{*}$. See Figure 4 . 


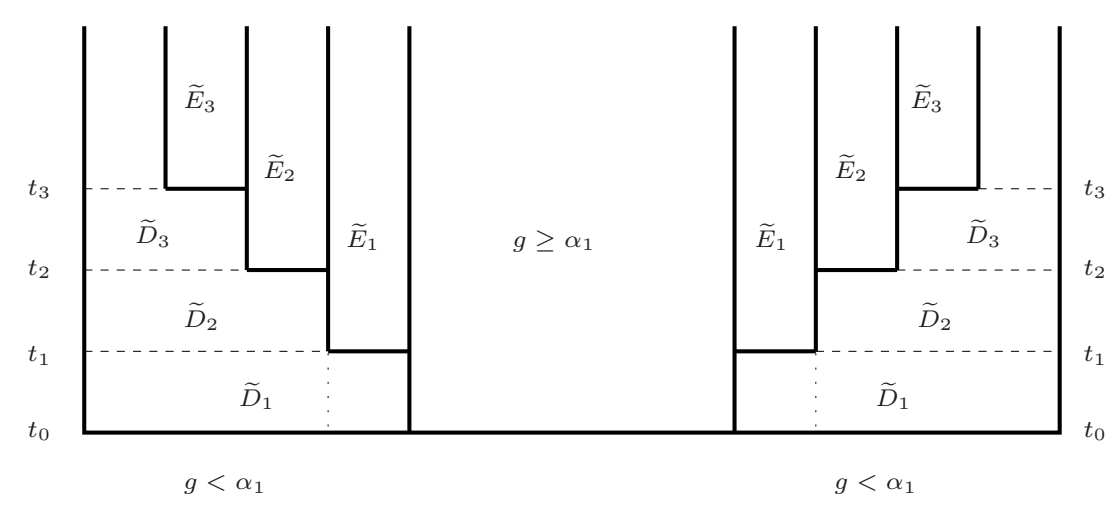

Figure 4. Parabolic box argument.

By the parabolic comparison principle over $\widetilde{D}_{1}$ we have

$$
P(t, x, D) \leq \frac{g(x)}{\alpha_{1}}+P\left(t, x, D_{1}\right) \quad \text { for }(t, x) \in \widetilde{D}_{1}=(0, \infty) \times D_{1} .
$$

Divide both sides by $g(x)$ and take the supremum over $\widetilde{E}_{1}$. Then (1.7) gives

$$
\begin{aligned}
q_{1} & \leq \frac{1}{\alpha_{1}}+\sup _{(t, x) \in \widetilde{E}_{1}} \frac{P\left(t, x, D_{1}\right)}{g(x)} \leq \frac{1}{\alpha_{1}}+\frac{A_{0}}{\alpha_{2}} \sup _{t \geq t_{1}} \exp \left(-\frac{A_{1} t}{w_{\eta}\left(D_{1}\right)^{2}}\right) \\
& \leq \frac{1}{\alpha_{1}}+\frac{A_{0}}{\alpha_{2}} \exp \left(-\frac{A_{1}\left(t_{1}-t_{0}\right)}{w_{\eta}\left(D_{1}\right)^{2}}\right) \leq A^{*} .
\end{aligned}
$$

Let $j \geq 2$. By the parabolic comparison principle over $\widetilde{D}_{j}$ we have

$$
P(t, x, D) \leq q_{j-1} g(x)+P\left(t-t_{j-1}, x, D_{j}\right) \quad \text { for }(t, x) \in \widetilde{D}_{j}=\left(t_{j-1}, \infty\right) \times D_{j} .
$$

Divide both sides by $g(x)$ and take the supremum over $\widetilde{E}_{j}$. In the same way as above, we obtain from (1.7) that

$$
q_{j} \leq q_{j-1}+\frac{A_{0}}{\alpha_{j+1}} \exp \left(-\frac{A_{1}\left(t_{j}-t_{j-1}\right)}{w_{\eta}\left(D_{j}\right)^{2}}\right) .
$$

Hence

$$
\begin{aligned}
q_{j} & \leq q_{1}+\sum_{j=2}^{\infty} \frac{A_{0}}{\alpha_{j+1}} \exp \left(-\frac{A_{1}\left(t_{j}-t_{j-1}\right)}{w_{\eta}\left(D_{j}\right)^{2}}\right) \\
& \leq \frac{1}{\alpha_{1}}+\sum_{j=1}^{\infty} \frac{A_{0}}{\alpha_{j+1}} \exp \left(-\frac{A_{1}\left(t_{j}-t_{j-1}\right)}{w_{\eta}\left(D_{j}\right)^{2}}\right)=A^{*} .
\end{aligned}
$$

The lemma follows.

Second, we choose $\alpha_{j}$ and $t_{j}$. Let us begin with a rather simple case. Suppose that $g(x) \geq A \delta_{D}(x)^{\alpha}$ for $x \in D$ with $\alpha>0$. Then

$$
\{x \in D: g(x)<t\} \subset\left\{x \in D: \delta_{D}(x) \leq(t / A)^{1 / \alpha}\right\} .
$$


Hence, if $D$ satisfies the CDC, or if $D$ is a John domain,

$$
w_{\eta}(g<t) \leq A t^{1 / \alpha} \text { for } t>0
$$

(Lemma 3.13 and Remark 3.16). In this case, we have the following sharp comparison between $P(t, x, D)$ and $g(x)$.

Lemma 4.2. Suppose (4.1) holds. If $t>0$, then

$$
P(t, x, D) \leq \frac{A}{t^{\alpha / 2}} g(x) \quad \text { for } x \in D .
$$

Proof. Let $t>0$ be fixed. Define $\alpha_{j}=2^{-j} t^{\alpha / 2}$ and $t_{j}=\left(1-(j+1)^{-1}\right) t$ for $j \geq 1$ and $t_{0}=0$. Then

$$
t_{j}-t_{j-1}=t\left(\frac{1}{j}-\frac{1}{j+1}\right) \geq \frac{t}{(j+1)^{2}}
$$

so that $t_{j} \uparrow t$ and

$$
\begin{aligned}
\frac{1}{\alpha_{1}}+\sum_{j=1}^{\infty} \frac{A_{0}}{\alpha_{j+1}} & \exp \left(-\frac{A_{1}\left(t_{j}-t_{j-1}\right)}{w_{\eta}\left(D_{j}\right)^{2}}\right) \\
& \leq 2 t^{-\alpha / 2}+A_{0} \sum_{j=1}^{\infty} 2^{j+1} t^{-\alpha / 2} \exp \left(-\frac{A_{1}(j+1)^{-2} t}{\left(A\left(2^{-j} t^{\alpha / 2}\right)^{1 / \alpha}\right)^{2}}\right) \\
& =\left\{2+A_{0} \sum_{j=1}^{\infty} 2^{j+1} \exp \left(-\frac{A_{1} 2^{2 j / \alpha}}{A^{2}(j+1)^{2}}\right)\right\} t^{-\alpha / 2} .
\end{aligned}
$$

Hence the required estimate follows from Lemma 4.1.

Now let us consider the general case when (1.8) holds. We have to choose $\alpha_{j}$ and $t_{j}$ more carefully.

Lemma 4.3. For each $\varepsilon>0$ there exist positive constants $A_{2}$ and $A_{3}$ depending only on $c_{\mathcal{L}}, n, \eta$ and $\varepsilon$ with the following property: Suppose (1.8) holds. If $t>0$, then

$$
P(t, x, D) \leq \frac{A_{2}}{m^{1+\varepsilon}} g(x) \quad \text { for } x \in D
$$

with $m>0$ being chosen so that (1.10) holds.

Proof. Let $\kappa=(1+\varepsilon)^{1 / 2}, \alpha_{j}=\exp \left(-\kappa^{j}\right)$ and

$$
A_{3}=\frac{A_{1}(\kappa-1)}{(\kappa+1) \kappa}
$$

Let $t>0$ be fixed. Choose $m \in(0,1)$ satisfying (1.10). Let $k$ be the positive integer such that $\alpha_{k} \leq m<\alpha_{k-1}$. Define

$$
t_{j}=\frac{\kappa+1}{A_{1}} \sum_{i=k+1}^{j} \kappa^{i} w_{\eta}\left(g<\alpha_{i}\right)^{2} \quad \text { for } j \geq k+1,
$$

and $t_{k}=0$. 
Since

$$
\int_{\alpha_{j}}^{\alpha_{j-1}} w_{\eta}(g<s)^{2} \frac{d s}{s} \geq w_{\eta}\left(g<\alpha_{j}\right)^{2} \int_{\alpha_{j}}^{\alpha_{j-1}} \frac{d s}{s}=\frac{\kappa-1}{\kappa} \kappa^{j} w_{\eta}\left(g<\alpha_{j}\right)^{2},
$$

it follows from (1.10) and $\alpha_{k} \leq m$ that

$$
\begin{aligned}
\lim _{j \rightarrow \infty} t_{j} & =\frac{\kappa+1}{A_{1}} \sum_{j=k+1}^{\infty} \kappa^{j} w_{\eta}\left(g<\alpha_{j}\right)^{2} \leq \frac{(\kappa+1) \kappa}{A_{1}(\kappa-1)} \sum_{j=k+1}^{\infty} \int_{\alpha_{j}}^{\alpha_{j-1}} w_{\eta}(g<s)^{2} \frac{d s}{s} \\
& =A_{3}^{-1} \int_{0}^{\alpha_{k}} w_{\eta}(g<s)^{2} \frac{d s}{s} \leq t .
\end{aligned}
$$

Observe that

$$
\frac{1}{\alpha_{j+1}} \exp \left(-\frac{A_{1}\left(t_{j}-t_{j-1}\right)}{w_{\eta}\left(g<\alpha_{j}\right)^{2}}\right)=\exp \left(\kappa^{j+1}-(\kappa+1) \kappa^{j}\right)=\exp \left(-\kappa^{j}\right) .
$$

Hence Lemma 4.1 (with slight modification) yields $P(t, x, D) \leq A^{*} g(x)$ for $x \in D$ with

$$
A^{*}=\frac{1}{\alpha_{k+1}}+\sum_{j=k+1}^{\infty} \frac{A_{0}}{\alpha_{j+1}} \exp \left(-\frac{A_{1}\left(t_{j}-t_{j-1}\right)}{w_{\eta}\left(D_{j}\right)^{2}}\right)=\exp \left(\kappa^{k+1}\right)+\sum_{j=k+1}^{\infty} \exp \left(-\kappa^{j}\right) .
$$

Since $m<\alpha_{k-1}=\exp \left(-\kappa^{k-1}\right)$, it follows that

$$
\exp \left(\kappa^{k+1}\right)=\left(\exp \left(\kappa^{k-1}\right)\right)^{\kappa^{2}}<\frac{1}{m^{\kappa^{2}}}=\frac{1}{m^{1+\varepsilon}}
$$

so that

$$
A^{*} \leq \frac{1}{m^{1+\varepsilon}}+\sum_{j=1}^{\infty} \exp \left(-\kappa^{j}\right) \leq \frac{A}{m^{1+\varepsilon}} .
$$

The lemma is proved.

Remark 4.4. We note that the decomposition in Lemma 4.1 does not break the boundary $\partial D$. More precisely, every $\partial D_{k}$ contains $\partial D$ except for a polar set, since $g(x) \rightarrow 0$ as $x \in D$ tends to a regular boundary point of $\partial D$ and since the set of all irregular boundary points forms a polar set. This is a big difference between the parabolic and elliptic box arguments.

\section{Proof of Theorem $\mathbf{1 . 9}$}

Let us begin with the following two general lemmas.

Lemma 5.1 (Proof of Theorem 1.2 in [12]). There exists a positive constant $A$ depending only on $c_{\mathcal{L}}$ and $n$ such that

$$
p(3 t, x, y) \leq A t^{-n / 2} P(t, x, D) P(t, y, D) \quad \text { for } x, y \in D .
$$


Proof. In view of $(1.25)$, we have $p(t, x, y) \leq p_{\mathbb{R}^{n}}(t, x, y) \leq A t^{-n / 2}$. Observe that the heat kernel is symmetric, i.e., $p(t, x, y)=p(t, y, x)$ for $x, y \in D$. Let $x, y, z \in D$. Then

$p(2 t, z, y)=\int_{D} p(t, z, w) p(t, w, y) d w \leq A t^{-n / 2} \int_{D} p(t, w, y) d w=A t^{-n / 2} P(t, y, D)$, so that $p(3 t, x, y)$ is bounded by

$$
\begin{aligned}
\int_{D} p(t, x, z) p(2 t, z, y) d z & \leq A t^{-n / 2} \int_{D} p(t, x, z) P(t, y, D) d z \\
& =A t^{-n / 2} P(t, x, D) P(t, y, D) .
\end{aligned}
$$

Lemma 5.2. We have

$$
\left\|\varphi_{D}\right\|_{\infty} \leq A \lambda_{D}^{n / 4}
$$

where $A$ depends only on $c_{\mathcal{L}}$ and $n$.

Proof. Observe that $e^{-\lambda_{D} t} \varphi_{D}(x)$ is a caloric function with vanishing lateral boundary values, so that

$$
e^{-\lambda_{D} t} \varphi_{D}(x)=\int_{D} p(t, x, y) \varphi_{D}(y) d y \leq\|p(t, x, \cdot)\|_{2}\left\|\varphi_{D}\right\|_{2}=\|p(t, x, \cdot)\|_{2} .
$$

By (1.25) we have

$$
\begin{aligned}
\int_{D} p(t, x, y)^{2} d y & \leq \int_{\mathbb{R}^{n}} \frac{A}{t^{n}} \exp \left(-2 A^{\prime} \frac{|x-y|^{2}}{t}\right) d y \\
& =\frac{A}{t^{n}}(\sqrt{t})^{n} \int_{\mathbb{R}^{n}} \exp \left(-2 A^{\prime}|z|^{2}\right) d z=A t^{-n / 2} .
\end{aligned}
$$

Hence $\varphi_{D}(x) \leq A t^{-n / 4} e^{\lambda_{D} t}$. Letting $t=1 / \lambda_{D}$ and taking the supremum for $x \in D$, we obtain the lemma.

We compare now the Green function $g(x)$ and the ground state $\varphi_{D}(x)$ near the boundary. In general, $g(x)$ is majorized by $\varphi_{D}(x)$ whenever it exists.

Lemma 5.3. Suppose (1.6) holds. Then

$$
g(x) \leq A \varphi_{D}(x) \quad \text { for } x \in D \backslash B\left(x_{0}, \delta_{D}\left(x_{0}\right) / 2\right),
$$

where $A>0$ depends only on $c_{\mathcal{L}}, D$ and $x_{0}$.

Proof. By Corollary 1.7 we have the ground state $\varphi_{D} \in L^{2}(D)$. Let us invoke the elliptic comparison principle. Since $-\mathcal{L} \varphi_{D}=\lambda_{D} \varphi_{D}>0$, it follows that $\varphi_{D}$ is superharmonic in $D$. Let $m_{0}=\inf _{\bar{B}\left(x_{0}, \delta_{D}\left(x_{0}\right) / 2\right)} \varphi_{D}(x)$. This is a positive quantity depending on $D$ and $x_{0}$. On the other hand, $g$ is bounded and harmonic in $D \backslash \bar{B}\left(x_{0}, \delta_{D}\left(x_{0}\right) / 2\right)$ and vanishes q.e. on $\partial D$, so that the comparison principle yields

$$
g(x) \leq \frac{M_{0}}{m_{0}} \varphi_{D}(x) \quad \text { for } x \in D \backslash B\left(x_{0}, \delta_{D}\left(x_{0}\right) / 2\right)
$$

with $M_{0}=\sup _{\partial B\left(x_{0}, \delta_{D}\left(x_{0}\right) / 2\right)} g$. 
Proof of Theorem 1.9. Combining Lemmas 4.3, 5.1 and 5.3, we obtain

$$
p(3 t, x, y) \leq A t^{-n / 2} m^{-2(1+\varepsilon)} g(x) g(y) \leq A t^{-n / 2} m^{-2(1+\varepsilon)} \varphi_{D}(x) \varphi_{D}(y)
$$

for $x, y \in D \backslash B\left(x_{0}, \delta_{D}\left(x_{0}\right) / 2\right)$, where $m$ satisfies (1.10). Since $\varphi_{D} \geq A>0$ on a compact subset of $D$, the same inequality holds for $x, y \in B\left(x_{0}, \delta_{D}\left(x_{0}\right) / 2\right)$. Replacing $t$ and $\varepsilon$ by $t / 3$ and $\varepsilon / 2$, respectively, we obtain

$$
p(t, x, y) \leq \frac{A}{m^{2+\varepsilon}} t^{-n / 2} \varphi_{D}(x) \varphi_{D}(y) \quad \text { for } x, y \in D
$$

which implies (1.9). The opposite inequality $p(t, x, y) \geq C_{t}^{\prime} \varphi_{D}(x) \varphi_{D}(y)$ holds by Proposition 2.2. Hence $D$ is IU. The theorem is proved.

Remark 5.4. The above proof says

$$
g(x) \approx \varphi_{D}(x) \text { for } x \in D \backslash B\left(x_{0}, \delta_{D}\left(x_{0}\right) / 2\right),
$$

where the constant of comparison depends on $c_{\mathcal{L}}, D$ and $x_{0}$.

Using Lemma 4.2 instead of Lemma 4.3, we obtain the following estimate of $C_{t}$.

Proposition 5.5. Let $g(x) \geq A \delta_{D}(x)^{\alpha}$ for $x \in D$ with $\alpha>0$. Suppose, either $D$ satisfies the $C D C$, or $D$ is a John domain. Then $C_{t}$ in (1.2) has an estimate $C_{t} \leq A t^{-n / 2-\alpha}$.

Remark 5.6. With the aid of the log-Sobolev inequality, Ouhabaz and Wang (Corollary $2.4(\mathrm{~b})$ in [33]) proved the same estimate of $C_{t}$ under an assumption including the Hardy inequality, which is related to the CDC (Theorems 1, 2, and Proposition 1 in [6]).

\section{Elliptic box argument. Proof of Theorem 1.11}

Let us state an elliptic counterpart of Lemma 4.1. This is essentially given in the proof of Theorem 1.3 in [5]. For completeness, and for the comparison with the parabolic box argument, we provide a proof. Let us begin by recalling an elliptic counterpart of (1.7). The following lemma is known in the case $\mathcal{L}=\Delta$. But, for completeness, we shall provide a proof in the appendix.

Lemma $\mathbf{E}$ (Proposition 2 in [1] and Lemma 1 in [2]). Denote by $\omega^{x}(E, D)$ the harmonic measure of $E$ in $D$, evaluated at $x$. Let $D$ be an open set, $x \in D$ and $R>0$. Then

$$
\omega^{x}(D \cap \partial B(x, R), D \cap B(x, R)) \leq A_{5} \exp \left(-\frac{A_{6} R}{w_{\eta}(D)}\right),
$$

where positive constants $A_{5}$ and $A_{6}$ depend only on $n, c_{\mathcal{L}}$ and $\eta>0$. 
Remark 6.1. Compare the exponent 1 of $w_{\eta}(D)$ in Lemma E and the exponent 2 of $w_{\eta}(D)$ in (1.7). This difference suggests that IU is a condition much weaker than the boundary Harnack principle.

Lemma 6.2 (Elliptic box argument). Suppose (1.13) holds. Let $K$ be a compact set intersecting $D$ and let $R>0$. Then

$$
\omega(D \cap \partial K, D \backslash K) \leq A_{R} g \quad \text { on }\{x \in D: \operatorname{dist}(x, K)>R\},
$$

where $A_{R}>0$ depends on $R, K$ and $D$.

Proof. For $r \geq 0$ we put $L(r)=\{x \in D: \operatorname{dist}(x, K)=r\}$ and $U(r)=\{x \in D$ : $\operatorname{dist}(x, K)>r\}$. Let $\omega_{0}=\omega(D \cap \partial K, D \backslash K)$. It is sufficient to show that

$$
\omega_{0} \leq A_{R} g \text { on } U(R) .
$$

The dependency of $R$ on $A_{R}$ has no significance for the GBHP, so we fix $\kappa=2$ and apply an argument similar to the proof of Lemma 4.3. We have from (1.13)

$$
\sum_{i=0}^{\infty} 2^{i} w_{\eta}\left(D_{i}\right)<\infty
$$

where $D_{i}=\left\{x \in D: g(x)<\exp \left(-2^{i}\right)\right\}$. We find a positive integer $k$ such that

$$
R_{\infty}:=\frac{3}{A_{6}} \sum_{i=k}^{\infty} 2^{i} w_{\eta}\left(D_{i}\right)<R,
$$

where $A_{6}>0$ is the constant in Lemma E. Let $R_{k-1}=0$ and let

$$
R_{j}=\frac{3}{A_{6}} \sum_{i=k}^{j} 2^{i} w_{\eta}\left(D_{i}\right)
$$

for $j \geq k$. Then $R_{j} \uparrow R_{\infty}<R$ and

$$
R_{j}-R_{j-1}=\frac{3}{A_{6}} 2^{j} w_{\eta}\left(D_{j}\right)
$$

for $j \geq k$. Put

$$
\begin{aligned}
& U_{j}=\left\{x \in U\left(R_{j-1}\right): 0<g(x)<\exp \left(-2^{j}\right)\right\}, \\
& T_{j}=\left\{x \in U\left(R_{j}\right): \exp \left(-2^{j+1}\right) \leq g(x)<\exp \left(-2^{j}\right)\right\}, \\
& q_{j}= \begin{cases}\sup _{T_{j}} \frac{\omega_{0}}{g} & \text { if } T_{j} \neq \emptyset, \\
0 & \text { if } T_{j}=\emptyset .\end{cases}
\end{aligned}
$$

See Figure 5. 


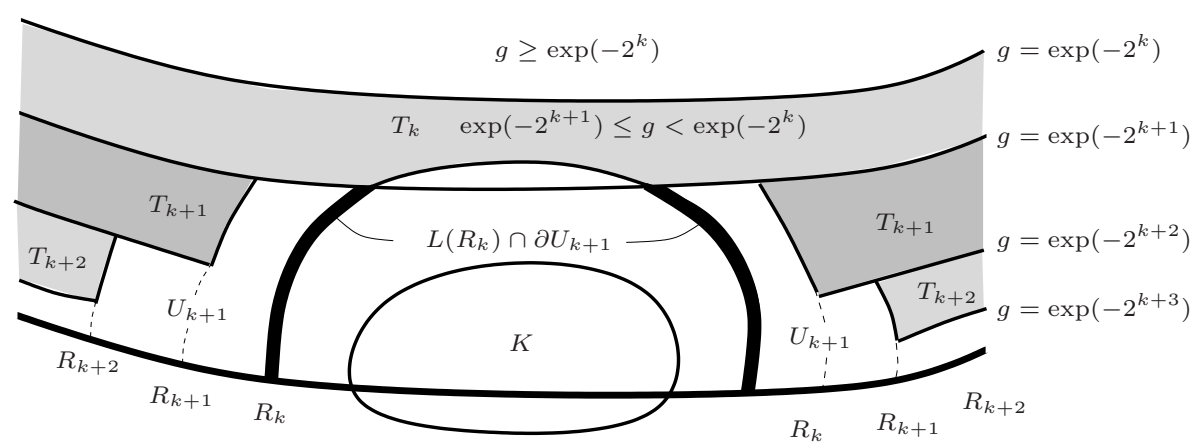

FiguRE 5. Elliptic box argument.

By definition, $q_{k} \leq \exp \left(2^{k+1}\right)$. Let $j>k$. Let us invoke the maximum principle over $U_{j}$. Observe that

$$
\begin{aligned}
D \cap \partial U_{j} & \subset\left\{x \in D \cap \partial U_{j}: g(x)=\exp \left(-2^{j}\right)\right\} \cup\left(L\left(R_{j-1}\right) \cap \partial U_{j}\right) \\
& \subset T_{j-1} \cup\left(L\left(R_{j-1}\right) \cap \partial U_{j}\right) .
\end{aligned}
$$

The maximum principle yields

$$
\omega_{0} \leq q_{j-1} g+\omega\left(L\left(R_{j-1}\right) \cap \partial U_{j}, U_{j}\right) \text { on } U_{j},
$$

so that

$$
q_{j}=\sup _{T_{j}} \frac{\omega_{0}}{g} \leq q_{j-1}+\exp \left(2^{j+1}\right) \sup _{T_{j}} \omega\left(L\left(R_{j-1}\right) \cap \partial U_{j}, U_{j}\right) .
$$

Let us estimate the harmonic measure on the right-hand side. Let $x \in T_{j}$. Then $B\left(x, R_{j}-R_{j-1}\right) \subset U\left(R_{j-1}\right)$ by definition. Hence the maximum principle yields

$$
\omega\left(L\left(R_{j-1}\right) \cap \partial U_{j}, U_{j}\right) \leq \omega\left(\partial B\left(x, R_{j}-R_{j-1}\right) \cap U_{j}, B\left(x, R_{j}-R_{j-1}\right) \cap U_{j}\right)
$$

on $B\left(x, R_{j}-R_{j-1}\right) \cap U_{j}$. Evaluating at $x$, and then applying Lemma E, we obtain

$$
\begin{aligned}
\omega^{x}\left(L\left(R_{j-1}\right)\right. & \left.\cap \partial U_{j}, U_{j}\right) \leq \omega^{x}\left(\partial B\left(x, R_{j}-R_{j-1}\right) \cap U_{j}, B\left(x, R_{j}-R_{j-1}\right) \cap U_{j}\right) \\
& \leq A_{5} \exp \left(-\frac{A_{6}\left(R_{j}-R_{j-1}\right)}{w_{\eta}\left(U_{j}\right)}\right) \leq A_{5} \exp \left(-\frac{A_{6}\left(R_{j}-R_{j-1}\right)}{w_{\eta}\left(D_{j}\right)}\right) .
\end{aligned}
$$

Hence (6.3) becomes

$$
q_{j} \leq q_{j-1}+A_{5} \exp \left(2^{j}\left(2-\frac{A_{6}\left(R_{j}-R_{j-1}\right)}{2^{j} w_{\eta}\left(D_{j}\right)}\right)\right)=q_{j-1}+A_{5} \exp \left(-2^{j}\right),
$$

where the equality follows from (6.2). Hence

$$
q_{j} \leq \exp \left(2^{k+1}\right)+A_{5} \sum_{j=k+1}^{\infty} \exp \left(-2^{j}\right)<\infty .
$$


With $A_{7}>0$ being the right-hand side we have

$$
\omega_{0} \leq A_{7} g \quad \text { on } \bigcup_{j=k}^{\infty} T_{j} .
$$

Since $U(R) \subset\left\{x: g(x) \geq \exp \left(-2^{k}\right)\right\} \cup \bigcup_{j=k}^{\infty} T_{j}$, it follows that

$$
\omega_{0} \leq \max \left\{A_{7}, \exp \left(2^{k}\right)\right\} g=A_{7} g \quad \text { on } U(R) .
$$

Thus (6.1) follows. The lemma is proved.

Proof of Theorem 1.11. Actually, the first inequality of (1.12) holds for an arbitrary domain $D$. Let $K$ and $F$ be disjoint compact sets such that $x_{0} \notin K \cup F$. We find a ball $B_{0}=B\left(x_{0}, r_{0}\right)$ such that $0<r_{0}<\min \left\{\delta_{D}\left(x_{0}\right)\right.$, $\left.\operatorname{dist}\left(x_{0}, K \cup F\right)\right\}$. Let $y \in D \cap F$. By the Harnack inequality we have $G(\cdot, y) \approx G\left(x_{0}, y\right)=g(y)$ on $\partial B_{0}$. Obviously, $g \leq A$ on $\partial B_{0}$. Compare $G(\cdot, y) / g(y)$ and $g$ on $D \backslash B_{0}$. Since the first is superharmonic and the second is harmonic, and since both are bounded near $\partial D$, say in $D \backslash\left(B_{0} \cup B\left(y, \delta_{D}(y) / 2\right)\right)$, and both vanish q.e. on $\partial D$, it follows from the maximum principle that

$$
\frac{G(\cdot, y)}{g(y)} \geq A g \quad \text { on } D \backslash B_{0}
$$

and, in particular,

$$
G(x, y) \geq A g(x) g(y) \quad \text { for } x \in D \cap K .
$$

For the second inequality of (1.12) we employ Lemma 6.2. Let $\operatorname{dist}(K, F)=3 R$ and put $K_{j}=\left\{z \in \mathbb{R}^{n}: \operatorname{dist}(z, K) \leq j R\right\}$ for $j=1,2$. Observe that

$$
A(K, F)=\sup _{z_{1} \in D \cap \partial K_{1}, z_{2} \in D \cap \partial K_{2}} G\left(z_{1}, z_{2}\right)<\infty .
$$

Fix $z \in D \cap \partial K_{2}$ for a moment. Then the maximum principle yields

$$
G(x, z) \leq A(K, F) \omega^{x}\left(D \cap \partial K_{1}, D \backslash \partial K_{1}\right) \quad \text { for } x \in D \cap K_{1} .
$$

Hence Lemma 6.2 implies that $G(x, z) \leq A g(x)$ for $x \in D \cap K$. Now fix $x \in D \cap K$. Then

$$
\sup _{z \in D \cap \partial K_{2}} G(x, z) \leq A g(x)
$$

so that the maximum principle yields

$$
G(x, y) \leq A g(x) \omega^{y}\left(D \cap \partial K_{2}, D \backslash \partial K_{2}\right) \quad \text { for } y \in D \backslash K_{2} .
$$

Lemma 6.2 says that

$$
\omega^{y}\left(D \cap \partial K_{2}, D \backslash \partial K_{2}\right) \leq A g(y) \text { for } y \in D \cap F,
$$

so that $G(x, y) \leq A g(x) g(y)$ as required. 


\section{Sufficient conditions of Orlicz type. Proof of Theorem 1.16}

Combining Theorems 1.9 and 1.11, Lemmas 3.9 and 3.10, we obtain the following corollary.

Corollary 7.1. The following statements hold:

(i) If $\int_{0}^{1}|\{x \in D: g(x)<t\}|^{2 / n} \frac{d t}{t}<\infty$, then $D$ is $I U$.

(ii) If $\int_{0}^{1}|\{x \in D: g(x)<t\}|^{1 / n} \frac{d t}{t}<\infty$, then $D$ satisfies the GBHP.

Let $s=\log (1 / t)$. Then $\{x \in D: g(x)<t\}=\{x \in D: \log (1 / g(x))>s\}$. For simplicity we write $|\{\log (1 / g)>s\}|$ for the Lebesgue measure of the set in the right-hand side. By a change of variable, we see that the first assumption of Corollary 7.1 (i) becomes

$$
\int_{0}^{\infty}|\{\log (1 / g)>s\}|^{2 / n} d s<\infty
$$

Proof of Theorem 1.16 (i). Let $n=2$. Then (7.1) becomes

$$
\int_{0}^{\infty}|\{\log (1 / g)>s\}| d s=\int_{\{x \in D: g(x)<1\}} \log (1 / g) d x<\infty .
$$

Hence if $\log _{+}(1 / g) \in L^{1}(D)$, then $D$ is IU.

For the remaining assertions of Theorem 1.16 we need an estimate of $\Phi$-integrals. The next lemma says that there is no harm in assuming that $\Phi(t)$ is strictly increasing.

Lemma 7.2. Let $\varphi(t)$ be a nondecreasing positive continuous function of $t>0$. Then there exists a strictly increasing positive continuous function $\Phi(t)$ such that $\varphi(t) \leq \Phi(t) \leq 2 \varphi(t)$.

Proof. Observe that

$$
\Phi(t)=\varphi(t)+\int_{0}^{t} e^{-\tau} \varphi(\tau) d \tau
$$

has the required properties.

In the rest of this section we assume that $\Phi(t)$ is strictly increasing and continuous and let $\Psi(t)$ be its inverse function.

Lemma 7.3. If $f$ is a positive measurable function on $D$, then

$$
\sum_{j=1}^{\infty} 2^{j}\left|\left\{f>\Psi\left(2^{j}\right)\right\}\right| \leq 2 \int_{D} \Phi(f) d x
$$


Proof. By definition,

$$
\begin{aligned}
\int_{D} \Phi(f) & d x=\int_{0}^{\infty}|\{f>t\}| d \Phi(t) \\
& \geq \int_{\Psi(1)}^{\infty}|\{f>t\}| d \Phi(t)=\sum_{j=1}^{\infty} \int_{\Psi\left(2^{j-1}\right)}^{\Psi\left(2^{j}\right)}|\{f>t\}| d \Phi(t) \\
& \geq \sum_{j=1}^{\infty}\left|\left\{f>\Psi\left(2^{j}\right)\right\}\right|\left(\Phi\left(\Psi\left(2^{j}\right)\right)-\Phi\left(\Psi\left(2^{j-1}\right)\right)\right)=\sum_{j=1}^{\infty}\left|\left\{f>\Psi\left(2^{j}\right)\right\}\right| 2^{j-1} .
\end{aligned}
$$

This gives the required inequality.

Proof of Theorem 1.16 (ii) and (iii). (ii) Let $n \geq 3$ and suppose $\Phi$ satisfies (1.16). Assume that $\log _{+}(1 / g) \in L^{\Phi}(D)$. Observe that

$$
\begin{aligned}
\int_{\Psi(1)}^{\infty}|\{\log (1 / g)>s\}|^{2 / n} d s & =\sum_{j=0}^{\infty} \int_{\Psi\left(2^{j}\right)}^{\Psi\left(2^{j+1}\right)}|\{\log (1 / g)>s\}|^{2 / n} d s \\
& \leq \sum_{j=0}^{\infty}\left|\left\{\log (1 / g)>\Psi\left(2^{j}\right)\right\}\right|^{2 / n}\left(\Psi\left(2^{j+1}\right)-\Psi\left(2^{j}\right)\right),
\end{aligned}
$$

which is less than or equal to

$$
\left(\sum_{j=0}^{\infty} 2^{j}\left|\left\{\log (1 / g)>\Psi\left(2^{j}\right)\right\}\right|\right)^{\frac{2}{n}}\left(\sum_{j=0}^{\infty}\left(2^{j}\right)^{2 /(2-n)}\left(\Psi\left(2^{j+1}\right)-\Psi\left(2^{j}\right)\right)^{n /(n-2)}\right)^{1-\frac{2}{n}}
$$

by Hölder's inequality. From Lemma 7.3 with $f=\log _{+}(1 / g)$ we have

$$
\sum_{j=0}^{\infty} 2^{j}\left|\left\{\log (1 / g)>\Psi\left(2^{j}\right)\right\}\right| \leq 2 \int_{D} \Phi\left(\log _{+}(1 / g)\right) d x<\infty .
$$

Since $n /(n-2)>1$, we have

$$
\left(\Psi\left(2^{j+1}\right)-\Psi\left(2^{j}\right)\right)^{n /(n-2)} \leq \Psi\left(2^{j+1}\right)^{n /(n-2)}-\Psi\left(2^{j}\right)^{n /(n-2)},
$$

so the second series in (7.2) is less than or equal to

$$
\sum_{j=0}^{\infty}\left(2^{j}\right)^{2 /(2-n)}\left[\Psi\left(2^{j+1}\right)^{n /(n-2)}-\Psi\left(2^{j}\right)^{n /(n-2)}\right] .
$$

Observe that

$$
\begin{aligned}
\int_{\Psi\left(2^{j}\right)}^{\Psi\left(2^{j+1}\right)}\left(\frac{t}{\Phi(t)}\right)^{2 /(n-2)} d t & \geq \Phi\left(\Psi\left(2^{j+1}\right)\right)^{2 /(2-n)} \int_{\Psi\left(2^{j}\right)}^{\Psi\left(2^{j+1}\right)} t^{2 /(n-2)} d t \\
& =\frac{n-2}{n}\left(2^{j+1}\right)^{2 /(2-n)}\left[\Psi\left(2^{j+1}\right)^{n /(n-2)}-\Psi\left(2^{j}\right)^{n /(n-2)}\right] .
\end{aligned}
$$

Taking the summation for $j \geq 0$, we obtain from (1.16) that (7.3) is convergent, and hence (7.2) is finite. Therefore (7.1) holds, and hence $D$ is IU. 
(iii) The proof is similar. Let $n \geq 2$ and suppose $\Phi$ satisfies (1.17). Put $f=\log _{+}(1 / g)$ and assume that $f \in L^{\Phi}(D)$. It suffices to observe that

$$
\begin{aligned}
& \int_{\Psi(1)}^{\infty}|\{f>s\}|^{1 / n} d s \\
& \leq\left(\sum_{j=0}^{\infty} 2^{j}\left|\left\{f>\Psi\left(2^{j}\right)\right\}\right|\right)^{1 / n}\left(\sum_{j=0}^{\infty}\left(2^{j}\right)^{1 /(1-n)}\left[\Psi\left(2^{j+1}\right)^{n /(n-1)}-\Psi\left(2^{j}\right)^{n /(n-1)}\right]\right)^{1-1 / n},
\end{aligned}
$$

and that

$$
\int_{\Psi\left(2^{j}\right)}^{\Psi\left(2^{j+1}\right)}\left(\frac{t}{\Phi(t)}\right)^{1 /(n-1)} d t \geq \frac{n-1}{n}\left(2^{j+1}\right)^{1 /(1-n)}\left[\Psi\left(2^{j+1}\right)^{n /(n-1)}-\Psi\left(2^{j}\right)^{n /(n-1)}\right] .
$$

If $\log _{+}(1 / g) \in L^{p}(D)$ with $p>n / 2$, then the constant $C_{t}$ in (1.2) can be easily estimated.

Proposition 7.4. If $\log _{+}(1 / g) \in L^{p}(D)$ with $p>n / 2$, then $C_{t}$ in (1.2) has an estimate $\log \left(C_{t}\right)=O\left(t^{n /(n-2 p)}\right)$ as $t \rightarrow 0$. In particular, if $p>n$, then $\lim _{t \rightarrow 0} t \log \left(C_{t}\right)=0$.

Proof. Observe that

$$
\left\|\log _{+}(1 / g)\right\|_{p}^{p}=\int_{0}^{\infty}|\{\log (1 / g)>s\}| d s^{p}<\infty .
$$

Let $0<m<1$ and put $M=\log (1 / m)$. By Lemmas 3.9 and 3.10,

$$
\left.\int_{0}^{m} w_{\eta}(g<s)^{2} \frac{d s}{s}=\int_{M}^{\infty} w_{\eta}(\log (1 / g)>s\}\right)^{2} d s \leq A \int_{M}^{\infty}|\{\log (1 / g)>s\}|^{2 / n} d s .
$$

If $n=2$, then

$$
\int_{M}^{\infty}|\{\log (1 / g)>s\}| d s \leq M^{1-p} \int_{M}^{\infty}|\{\log (1 / g)>s\}| s^{p-1} d s \leq \frac{M^{1-p}}{p}\left\|\log _{+}(1 / g)\right\|_{p}^{p},
$$

so that we can choose $\log (1 / m)=A t^{1 /(1-p)}$ in (1.10), and hence $\log \left(C_{t}\right) \leq$ $\log \left(A_{2} / m^{2+\varepsilon}\right)+A \log (1 / t) \leq A t^{n /(n-2 p)}$ with $n=2$. If $n \geq 3$, then the Hölder inequality gives

$$
\begin{aligned}
\int_{M}^{\infty} \mid & \left.\{\log (1 / g)>s\}\right|^{2 / n} d s \\
& \leq\left(\int_{M}^{\infty}|\{\log (1 / g)>s\}| s^{p-1} d s\right)^{2 / n}\left(\int_{M}^{\infty} s^{2(p-1) /(2-n)} d s\right)^{1-2 / n} \\
& \leq A\left\|\log _{+}(1 / g)\right\|_{p}^{2 p / n} M^{(n-2 p) / n}
\end{aligned}
$$

so that we can choose $\log (1 / m)=A t^{n /(n-2 p)}$ in (1.10), and hence $\log \left(C_{t}\right) \leq$ $\log \left(A_{2} / m^{2+\varepsilon}\right)+A \log (1 / t) \leq A t^{n /(n-2 p)}$ as well. 


\section{Sufficient conditions in terms of quasihyperbolic metric. Proof of Theorem 1.23}

Let us give elementary estimates of some integrals. In view of Lemma 7.2 we may assume that $\Phi(t)$ is a strictly increasing positive function of $t>0$ and let $\Psi(t)$ be its inverse function.

Lemma 8.1. Let $\Phi(t)$ and $\Psi(t)$ be as above. Then the following inequalities hold:

$$
\begin{aligned}
& \int_{\Phi(1)}^{\infty} \frac{d t}{\Psi(t)} \leq 4 \int_{2}^{\infty} \Phi(t) \frac{d t}{t^{2}} \quad \text { and } \quad \int_{\Psi(2)}^{\infty} \Phi(t) \frac{d t}{t^{2}} \leq 4 \int_{1}^{\infty} \frac{d t}{\Psi(t)} \\
& \int_{\Phi(1)}^{\infty} \frac{d t}{\Psi(t)^{2}} \leq \frac{32}{3} \int_{2}^{\infty} \Phi(t) \frac{d t}{t^{3}} \quad \text { and } \quad \int_{\Psi(2)}^{\infty} \Phi(t) \frac{d t}{t^{3}} \leq 2 \int_{1}^{\infty} \frac{d t}{\Psi(t)^{2}} .
\end{aligned}
$$

Proof. Let $j$ be an integer. Then

$$
\begin{aligned}
& \int_{\Phi\left(2^{j-1}\right)}^{\Phi\left(2^{j}\right)} \frac{d t}{\Psi(t)} \leq \frac{1}{\Psi\left(\Phi\left(2^{j-1}\right)\right)} \int_{\Phi\left(2^{j-1}\right)}^{\Phi\left(2^{j}\right)} d t \leq \frac{\Phi\left(2^{j}\right)}{2^{j-1}} \\
& \int_{2^{j}}^{2^{j+1}} \Phi(t) \frac{d t}{t^{2}} \geq \Phi\left(2^{j}\right) \int_{2^{j}}^{2^{j+1}} \frac{d t}{t^{2}}=\frac{\Phi\left(2^{j}\right)}{2^{j+1}}
\end{aligned}
$$

so that

$$
\int_{\Phi\left(2^{j-1}\right)}^{\Phi\left(2^{j}\right)} \frac{d t}{\Psi(t)} \leq 4 \int_{2^{j}}^{2^{j+1}} \Phi(t) \frac{d t}{t^{2}}
$$

Summing up the above inequality for $j \geq 1$, we obtain the first inequality of (8.1). Similarly, we have

$$
\int_{\Psi\left(2^{j}\right)}^{\Psi\left(2^{j+1}\right)} \Phi(t) \frac{d t}{t^{2}} \leq \Phi\left(\Psi\left(2^{j+1}\right)\right)\left(\frac{1}{\Psi\left(2^{j}\right)}-\frac{1}{\Psi\left(2^{j+1}\right)}\right) \leq 4 \frac{2^{j-1}}{\Psi\left(2^{j}\right)} \leq 4 \int_{2^{j-1}}^{2^{j}} \frac{d t}{\Psi(t)} .
$$

Summing for $j \geq 1$, we obtain the second inequality of (8.1). We can prove (8.2) in a similar manner. Details are left to the reader.

Proof of Theorem 1.23. Let $\Phi(t)$ and $\Psi(t)$ be as above. We assume that $D$ satisfies the $\mathrm{QHB}(\Phi)$ condition. Observe that (1.20) is equivalent to

$$
\Psi\left(k_{D}\left(x, x_{0}\right)\right) \leq \frac{\delta_{D}\left(x_{0}\right)}{\delta_{D}(x)} \text { for all } x \in D .
$$

Let us estimate the capacitary width of the set $\{x \in D: g(x)<t\}$. For simplicity we write $\{g(x)<t\}$ for this set; and use the same convention for sets given by conditions on $x$. Observe from (1.15) that

$$
\begin{aligned}
\{g(x)<t\} & \subset\left\{k_{D}\left(x, x_{0}\right)>A \log (1 / t)\right\} \\
& \subset\left\{\Psi\left(k_{D}\left(x, x_{0}\right)\right)>\Psi(A \log (1 / t))\right\} \subset\left\{\delta_{D}(x)<\frac{\delta_{D}\left(x_{0}\right)}{\Psi(A \log (1 / t))}\right\} .
\end{aligned}
$$

Hence

$$
w_{\eta}(g<t)=w_{\eta}(\{g(x)<t\}) \leq \frac{A}{\Psi(A \log (1 / t))}
$$


by Lemma 3.13. Therefore,

$$
\int_{0} w_{\eta}(g<t) \frac{d t}{t} \leq \int_{0} \frac{A}{\Psi(A \log (1 / t))} \frac{d t}{t} \quad \text { and } \int_{0} w_{\eta}(g<t)^{2} \frac{d t}{t} \leq \int_{0} \frac{A}{\Psi(A \log (1 / t))^{2}} \frac{d t}{t} .
$$

By the change of variable $A \log (1 / t)=s$ we have

$$
\int_{0} \frac{A}{\Psi(A \log (1 / t))} \frac{d t}{t}=\int^{\infty} \frac{A}{\Psi(s)} d s \quad \text { and } \quad \int_{0} \frac{A}{\Psi(A \log (1 / t))^{2}} \frac{d t}{t}=\int^{\infty} \frac{A}{\Psi(s)^{2}} d s .
$$

We see from Lemma 8.1 that these integrals converge if (1.22) and (1.21) hold, respectively. Hence the theorem follows from Theorems 1.9 and 1.11.

If $D$ satisfies the $\mathrm{QHB}(\alpha)$ and the $\mathrm{CDC}$, then the constant $C_{t}$ in (1.2) can be easily estimated.

Proposition 8.2. If $D$ satisfies the $C D C$ and the $Q H B(\alpha)$ with $\alpha<2$, then $C_{t}$ in (1.2) has an estimate $\log \left(C_{t}\right)=O\left(t^{\alpha /(\alpha-2)}\right)$ as $t \rightarrow 0$. In particular, if $\alpha<1$, then $\lim _{t \rightarrow 0} t \log \left(C_{t}\right)=0$.

Proof. In view of (1.15), the $\mathrm{QHB}(\alpha)$, the CDC and Lemma 3.13

$$
w_{\eta}(\log (1 / g)>s) \leq w_{\eta}\left(k_{D}\left(\cdot, x_{0}\right)>A s\right) \leq w_{\eta}\left(\delta_{D}(x)<A s^{-1 / \alpha}\right) \leq A s^{-1 / \alpha} .
$$

Let $0<m<1$ and put $M=\log (1 / m)$. Then

$$
\int_{0}^{m} w_{\eta}(g<s)^{2} \frac{d s}{s}=\int_{M}^{\infty} w_{\eta}(\log (1 / g)>s)^{2} d s \leq A \int_{M}^{\infty} s^{-2 / \alpha} d s=\frac{A}{2 / \alpha-1} M^{(\alpha-2) / \alpha} .
$$

Hence we can choose $\log (1 / m)=A t^{\alpha /(\alpha-2)}$ in $(1.10)$, so that $\log \left(C_{t}\right) \leq \log \left(A_{2} / m^{2+\varepsilon}\right)$ $+A \log (1 / t) \leq A t^{\alpha /(\alpha-2)}$.

\section{Domains above the graph of a function. Proof of Theo- rem 1.26}

We assume that $f\left(x^{\prime}\right)<-a$ for some $a, 0<a<1$, and let $x_{0}$ be the origin. First we prove Proposition 1.25, providing a relationship between $D_{f}$ with $f \in L^{p}\left(B^{\prime}(0, R)\right)$ and the quasihyperbolic boundary condition.

Proof of Proposition 1.25. Let $x_{0}=(0, \ldots, 0)$. It is easy to see that

$$
k_{D}\left(x, x_{0}\right) \leq A \log \frac{\delta_{D}\left(x_{0}\right)}{\delta_{D}(x)}+A \leq A \delta_{D}(x)^{(1-n-p) / p}+A
$$

for $x \in D$ with $x_{n} \geq-a / 2$. Let $x \in D$ with $x_{n}<-a / 2$. Since $B\left(x, \delta_{D}(x)\right) \subset D$, it follows from geometry that

$$
f\left(y^{\prime}\right) \leq x_{n} \quad \text { for } y^{\prime} \in B^{\prime}\left(x^{\prime}, \delta_{D}(x)\right) .
$$


Hence

$$
A \delta_{D}(x)^{n-1}\left|x_{n}\right|^{p} \leq \int_{B^{\prime}\left(x^{\prime}, \delta_{D}(x)\right)}\left|f\left(y^{\prime}\right)\right|^{p} d y^{\prime} \leq \int_{B^{\prime}(0, R)}\left|f\left(y^{\prime}\right)\right|^{p} d y^{\prime}<\infty,
$$

so that $\left|x_{n}\right| \leq A \delta_{D}(x)^{(1-n) / p}$. Let $x^{*}=\left(x^{\prime}, 0\right)$ and let $\gamma$ be the line segment connecting $x$ and $x^{*}$. Since the distance between $x^{*}$ and $B^{\prime}(0, R) \times\{1\}$ (the top of $D$ ) is equal to 1 , it follows that

$$
\delta_{D}\left(x^{*}\right) \geq \operatorname{dist}(\gamma, \partial D)=\min \left\{\delta_{D}(x), 1\right\},
$$

so that $k_{D}\left(x, x^{*}\right) \leq A \delta_{D}(x)^{(1-n-p) / p}+A$. It follows from (9.1) with $x^{*}$ in place of $x$ that

$$
k_{D}\left(x, x_{0}\right) \leq k_{D}\left(x, x^{*}\right)+k_{D}\left(x^{*}, x_{0}\right) \leq A \delta_{D}(x)^{(1-n-p) / p}+A .
$$

Thus $D$ satisfies the $\mathrm{QHB}((p+n-1) / p)$ condition.

The above proposition says that if $f \in L^{p}\left(B^{\prime}(0, R)\right)$, then $D_{f}$ is a twisted $L^{p}$-domain in the sense of Bass-Burdzy (Definition 2.2 in [12]). Hence, if $p>$ $n-1$, then $D_{f}$ is IU under the additional assumption of the CDC (Theorem A). It is a hard problem to dispense with the CDC. Let us illustrate the difficulty in case $n \geq 3$. Take an upper semicontinuous function $f\left(x^{\prime}\right) \in L^{p}\left(B^{\prime}(0, R)\right)$ with $f\left(x^{\prime}\right) \leq-a$. Let $E^{\prime}$ be a countable subset of $B^{\prime}(0, R)$ with no accumulation points in $B^{\prime}(0, R)$ and put

$$
\tilde{f}\left(x^{\prime}\right)=\left\{\begin{array}{cl}
-a & \text { if } x^{\prime} \in E^{\prime} \\
f\left(x^{\prime}\right) & \text { if } x^{\prime} \in B^{\prime}(0, R) \backslash E^{\prime}
\end{array}\right.
$$

Then $\tilde{f}$ is an upper semicontinuous function in $L^{p}\left(B^{\prime}(0, R)\right)$ such that $\tilde{f}=f$ a.e. in $B^{\prime}(0, R)$. Since $E^{\prime} \times \mathbb{R}$ is a polar set, it follows that $\Omega=\left\{\left(x^{\prime}, x_{n}\right): x^{\prime} \in\right.$ $\left.B^{\prime}(0, R), f\left(x^{\prime}\right)<x_{n}<1\right\}$ and $\tilde{\Omega}=\left\{\left(x^{\prime}, x_{n}\right): x^{\prime} \in B^{\prime}(0, R), \tilde{f}\left(x^{\prime}\right)<x_{n}<1\right\}$ share many properties such as volume, and yet they have completely different nature. For instance, $\Omega \backslash \tilde{\Omega}$ consists of irregular boundary points of $\tilde{\Omega}$ and the quasihyperbolic metric $k_{\tilde{\Omega}}$ diverges there.

In order to overcome the difficulty, Bass-Burdzy [12] established their Lemma 2.4. That was our motivation of the previous paper [5], where we proved an extended Harnack inequality with an exceptional set. Let us state the result as Theorem 9.2 below, adapted for the present setting. The same result holds for $\mathcal{L}$-harmonic functions by uniform ellipticity. See also (1.4).

Let us introduce regularized reduced functions, which are closely related to capacity and capacitary width. See Sections $5.3-7$ in [7] for the case $\mathcal{L}=\Delta$. Let $U$ be an open set. For $E \subset U$ and a nonnegative function $u$ on $E$, we define the reduced function ${ }^{U} \mathbf{R}_{u}^{E}$ by

$$
{ }^{U} \mathbf{R}_{u}^{E}(x)=\inf \{v(x): v \geq 0 \text { is superharmonic in } U \text { and } v \geq u \text { on } E\} \quad \text { for } x \in U \text {. }
$$

The lower semicontinuous regularization of ${ }^{U} \mathbf{R}_{u}^{E}$ is called the regularized reduced function or balayage and is denoted by ${ }^{U} \widehat{\mathbf{R}}_{u}^{E}$. It is known that ${ }^{U} \widehat{\mathbf{R}}_{u}^{E}$ is a nonnegative superharmonic function, ${ }^{U} \widehat{\mathbf{R}}_{u}^{E} \leq{ }^{U} \mathbf{R}_{u}^{E}$ on $U$ and the equality sign holds q.e. 
on $U$. If $u$ is a nonnegative superharmonic function on $U$, then ${ }^{U} \widehat{\mathbf{R}}_{u}^{E} \leq u$ on $U$. By the maximum principle ${ }^{U} \widehat{\mathbf{R}}_{u}^{E}$ is nondecreasing with respect to $U$ and $E$. If $u$ is the constant function 1, then ${ }^{U} \widehat{\mathbf{R}}_{1}^{E}(x)$ is the probability of hitting $E$ before leaving $U$ when the diffusion starts at $x$. Reduced functions and capacity are closely related. The following lemma is known in case $\mathcal{L}=\Delta$. But, for completeness, we shall provide a proof in the appendix.

Lemma $\mathbf{F}$ (Lemmas 4.6 and 4.7 in [5]). Let $0<r<R$. If $E$ is a compact subset of $B(x, R)$, then

$$
\inf _{\bar{B}(x, r)} B(x, R) \widehat{\mathbf{R}}_{1}^{E} \leq \frac{\operatorname{Cap}_{B(x, R)}^{\mathcal{L}}(E)}{\operatorname{Cap}_{B(x, R)}^{\mathcal{L}}(\bar{B}(x, r))} .
$$

Moreover, if $E \subset \bar{B}(x, r)$, then

$$
\frac{\operatorname{Cap}_{B(x, R)}^{\mathcal{L}}(E)}{\operatorname{Cap}_{B(x, R)}^{\mathcal{L}}(\bar{B}(x, r))} \leq A \inf _{\bar{B}(x, r)} B(x, R) \widehat{\mathbf{R}}_{1}^{E},
$$

where $A>1$ depends only on $n, c_{\mathcal{L}}$ and $r / R$.

Reduced functions have a lower estimate in terms of Lebesgue measure.

Lemma 9.1. Let $\tau>1$. Then

$$
{ }^{B(x, \tau r)} \widehat{\mathbf{R}}_{1}^{E}(x) \geq A \frac{|E|}{|B(x, r)|} \text { for every Borel set } E \subset B(x, r),
$$

where $A$ depends only on $\tau, c_{\mathcal{L}}$ and $n$.

Proof. In view of (1.4), Lemmas F and 3.8, we have

$$
{ }_{B(x, \tau r)} \widehat{\mathbf{R}}_{1}^{E}(x) \geq A \frac{\operatorname{Cap}_{B(x, \tau r)}^{\mathcal{L}}(E)}{\operatorname{Cap}_{B(x, \tau r)}^{\mathcal{L}}(B(x, r))} \approx \frac{\operatorname{Cap}_{B(x, \tau r)}(E)}{\operatorname{Cap}_{B(x, \tau r)}(B(x, r))} \geq A \frac{|E|}{|B(x, r)|} .
$$

Theorem 9.2 (Theorem 5.5 in [5]). Let $A_{8}>1$ and $\tau>1$. Let $\left\{B\left(x_{j}, r_{j}\right)\right\}_{j=0}^{J}$ be a chain of balls satisfying the following:

(i) If $1 \leq j \leq J$, then

$$
\begin{aligned}
& A_{8}^{-1} r_{j-1} \leq r_{j} \leq A_{8} r_{j-1}, \\
& B\left(x_{j-1}, r_{j-1}\right) \cap B\left(x_{j}, r_{j}\right) \text { includes a ball of radius greater than } A_{8}^{-1} r_{j}, \\
& B\left(x_{j}, r_{j}\right) \backslash B\left(x_{j-1}, r_{j-1}\right) \text { includes a ball of radius greater than } A_{8}^{-1} r_{j} .
\end{aligned}
$$

(ii) If $|i-j| \geq 2$, then $B\left(x_{i}, \tau r_{i}\right) \cap B\left(x_{j}, \tau r_{j}\right)=\emptyset$.

Then there exist positive constants $\varepsilon_{0}<1$ and $A_{9}>1$ depending only on $n$, $A_{8}$, and $\tau$ with the following property: suppose that a closed set $E$ satisfies $E \cap$ $B\left(x_{J}, r_{J}\right)=\emptyset$,

$$
\begin{aligned}
& B\left(x_{0}, \tau r_{0}\right) \widehat{\mathbf{R}}_{1}^{E \cap B\left(x_{0}, r_{0}\right)}\left(x_{0}\right)<\varepsilon_{0}, \\
& \frac{\operatorname{Cap}_{B\left(x_{j}, \tau r_{j}\right)}\left(E \cap B\left(x_{j}, r_{j}\right)\right)}{\operatorname{Cap}_{B\left(x_{j}, \tau r_{j}\right)}\left(B\left(x_{j}, r_{j}\right)\right)}<\varepsilon_{0} \quad \text { for } j=1, \ldots, J-1 .
\end{aligned}
$$


Then

$$
\frac{h\left(x_{J}\right)}{h\left(x_{0}\right)} \leq \exp \left(A_{9} J\right),
$$

whenever $h$ is a positive harmonic function in $B\left(x_{0}, r_{0}\right) \cup \cdots \cup B\left(x_{J}, r_{J}\right) \backslash E$.

The intuitive meaning of the theorem is as follows: The Harnack type inequality (9.2) holds if the exceptional set $E$ has

(i) uniformly small, measured by capacity, intersection with intermediate balls $B\left(x_{j}, r_{j}\right), 1 \leq j \leq J-1$

(ii) no intersection with the last ball $B\left(x_{J}, r_{J}\right)$,

(iii) small intersection with the first ball $B\left(x_{0}, r_{0}\right)$ influencing little the value at $x_{0}$ of the regularized reduced function.

A chain of balls $\left\{B\left(x_{j}, r_{j}\right)\right\}_{j=0}^{J}$ satisfying (i) and (ii) of Theorem 9.2 is referred to as a Harnack simple chain. We can easily construct a Harnack simple chain for a domain above a graph.

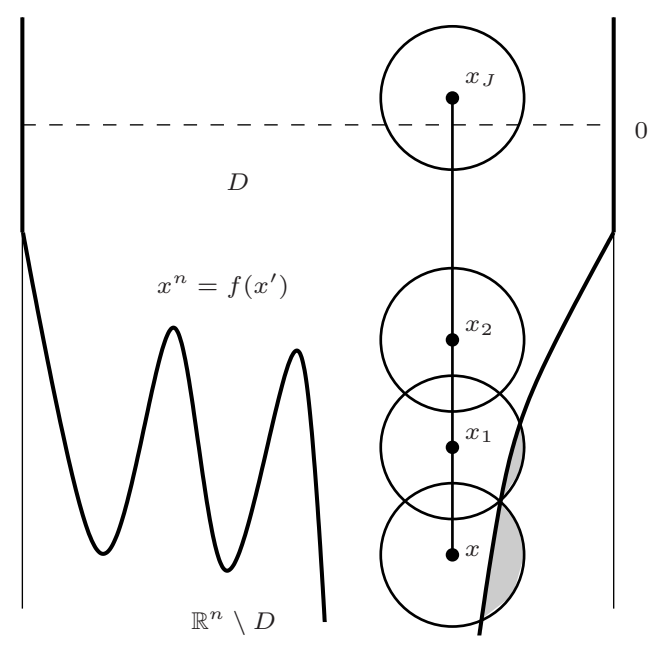

Figure 6. A domain above a graph and a Harnack simple chain with exceptional set.

Let $0<a \leq R$ and let $f\left(x^{\prime}\right)$ be an upper semicontinuous function of $x^{\prime} \in$ $B^{\prime}(0, R)$ such that $f<-a$ on $B^{\prime}(0, R)$. To distinguish a sequence of points and the component of a point, we write $x^{n}$ for the $n$-th component of a point $x$ in this paragraph. Consider the domain

$$
D=D_{f}=\left\{x=\left(x^{\prime}, x^{n}\right) \in \mathbb{R}^{n}:\left|x^{\prime}\right|<R, f\left(x^{\prime}\right)<x^{n}<1\right\}
$$

above the graph of $f$. Take a point $x=\left(x^{\prime}, x^{n}\right) \in D$ with $x^{n}<-a / 2$. Let $0<\rho<a / 4$ and $1<\tau \leq 2$. We place a sequence of points $x_{j}=\left(x^{\prime}, x^{n}+\tau \rho j\right)$ 
above the point $x=\left(x^{\prime}, x^{n}\right)$. Observe that

$$
\left|x_{i}-x_{j}\right|=|i-j| \tau \rho .
$$

Hence, if $|i-j| \geq 2$, then $\left|x_{i}-x_{j}\right| \geq 2 \tau \rho$, i.e., $B\left(x_{i}, \tau \rho\right) \cap B\left(x_{j}, \tau \rho\right)=\emptyset$. We also see that $B\left(x_{j-1}, \rho\right) \cap B\left(x_{j}, \rho\right)$ contains an open ball with radius $(1-\tau / 2) \rho$; and that $B\left(x_{j},(\tau-1) \rho\right) \subset B\left(x_{j}, \rho\right) \backslash B\left(x_{j-1}, \rho\right)$. Let $J$ be the integer such that $\tau \rho(J-1)<\left|x^{n}\right| \leq \tau \rho J$. Then $x_{J}^{n}=x^{n}+\tau \rho J$ satisfies $0 \leq x_{J}^{n}<\tau \rho<a / 2<1 / 2$, so that $B^{\prime}\left(x^{\prime}, R-\left|x^{\prime}\right|\right) \times\left(x_{J}^{n}-a / 2, x_{J}^{n}+a / 2\right) \subset D$. Hence, if $\left|x^{\prime}\right| \leq R-\rho$, then $\delta_{D}\left(x_{J}\right) \geq \min \left\{R-\left|x^{\prime}\right|, a / 2\right\} \geq \rho$, so that $\left\{B(x, \rho), B\left(x_{1}, \rho\right), \ldots, B\left(x_{J}, \rho\right)\right\}$ is a Harnack simple chain such that

$$
J \leq A\left|x^{n}\right| / \rho \text { and } B\left(x_{J}, \rho\right) \subset D .
$$

See Figure 6.

We show that if $B(x, 2 r) \widehat{\mathbf{R}}_{1}^{B(x, r) \backslash D}(x)$ is sufficiently small, Theorem 9.2 is applicable to the Harnack simple chain $\left\{B(x, \rho), B\left(x_{1}, \rho\right), \ldots, B\left(x_{J}, \rho\right)\right\}$ with $\rho=r / 2$.

Lemma 9.3. Let $a, R, f$ and $D$ be as above and let $\varepsilon_{0}$ be as in Theorem 9.2. Then there exists a positive constant $\eta_{0}$ depending only on $c_{\mathcal{L}}$ and $n$ such that if $x=\left(x^{\prime}, x_{n}\right) \in D$ and if $B(x, 2 r) \widehat{\mathbf{R}}_{1}^{B(x, r) \backslash D}(x)<\eta_{0}$, then

(i) $\left|x^{\prime}\right| \leq R-\rho$,

(ii) $\frac{\operatorname{Cap}_{B\left(x_{j}, \tau \rho\right)}\left(B\left(x_{j}, \rho\right) \backslash D\right)}{\operatorname{Cap}_{B\left(x_{j}, \tau \rho\right)}\left(B\left(x_{j}, \rho\right)\right)}<\varepsilon_{0}$ for $j=1, \ldots, J-1$,

where $\rho=r / 2$.

Proof. Observe that $D$ is included in the cylinder $W=B^{\prime}(0, R) \times(-\infty, 1)$. Let $\rho=r / 2$. An elementary geometrical argument gives

$$
\frac{|B(x, r) \backslash W|}{|B(x, r)|}>c_{1} \quad \text { if }\left|x^{\prime}\right|>R-\rho
$$

with $c_{1}>0$ depending only on the dimension $n$. On the other hand, we have from Lemma 9.1

$$
{ }^{B(x, 2 r)} \widehat{\mathbf{R}}_{1}^{B(x, r) \backslash D}(x) \geq A \frac{|B(x, r) \backslash W|}{|B(x, r)|} .
$$

Hence (i) follows with $\eta_{0} \leq A c_{1}$. We have

$$
{ }^{B(x, 2 r)} \widehat{\mathbf{R}}_{1}^{B(x, r) \backslash D}(x) \geq{ }^{B(x, \tau \rho)} \widehat{\mathbf{R}}_{1}^{B(x, \rho) \backslash D}(x) \geq A \frac{\operatorname{Cap}_{B(x, \tau \rho)}(B(x, \rho) \backslash D)}{\operatorname{Cap}_{B(x, \tau \rho)}(B(x, \rho))}
$$

by the monotonicity of $B(x, 2 r) \widehat{\mathbf{R}}_{1}^{B(x, r) \backslash D}(x)$ in $r$ and Lemma $\mathrm{F}$. In view of the geometric nature and translation invariance of capacity, we see that the right-hand side is greater than or equal to

$$
A \frac{\operatorname{Cap}_{B\left(x_{j}, \tau \rho\right)}\left(B\left(x_{j}, \rho\right) \backslash D\right)}{\operatorname{Cap}_{B\left(x_{j}, \tau \rho\right)}\left(B\left(x_{j}, \rho\right)\right)}
$$

for $j=1, \ldots, J-1$. Hence (ii) follows with $\eta_{0} \leq A \varepsilon_{0}$. The lemma follows. 
Lemma 9.4. Let $a, R, f$ and $D$ be as above. Let $\varphi(t)$ be a positive nondecreasing function of $t>0$ and let $\Phi(t)=(t \varphi(t))^{n-1}$. Suppose $f \in L^{\Phi}\left(B^{\prime}(0, R)\right)$. Then there exist positive constants $\eta, T<1, A_{10}$ and $A_{11}$ with the following property: let $0<t<T$ and put $s=\log (1 / t)$; if $x \in D$ and ${ }^{B(x, 2 r)} \widehat{\mathbf{R}}_{1}^{B(x, r) \backslash D}(x)<\eta$ with

$$
r^{2} s \varphi\left(A_{10} r s\right) \geq A_{11},
$$

then $g(x) \geq t$.

Remark 9.5. The hypothesis of this lemma allows $r>\delta_{D}(x)$. Hence the usual Harnack inequality is not enough to prove the lemma.

Proof of Lemma 9.4. Let $x \in D$. First, consider the case when $x_{n}>-a / 2$. Let $V=\left\{y \in D: y_{n}>-a\right\}$. Since $V$ coincides with the Lipschitz domain $B^{\prime}(0, R) \times$ $(-a, 1)$, the estimate $(1.18)$ is available, and hence, by (1.14),

$$
g(x) \geq A \delta_{D}(x)^{\alpha}
$$

for some $\alpha>0$. Suppose $g(x)<t$. Then $\delta_{D}(x) \leq A t^{1 / \alpha}$, so that, by the monotonicity of reduced functions,

$$
{ }^{B\left(x, 4 \delta_{D}(x)\right)} \widehat{\mathbf{R}}_{1}^{B\left(x, 2 \delta_{D}(x)\right) \backslash D}(x) \leq{ }^{B\left(x, 4 A t^{1 / \alpha}\right)} \widehat{\mathbf{R}}_{1}^{B\left(x, 2 A t^{1 / \alpha}\right) \backslash D}(x) .
$$

Moreover, it $t$ is small, then $\delta_{D}(x)<a / 2$, so that $B\left(x, \delta_{D}(x)\right)$ touches $\partial D$ at $x^{*} \in$ $\partial D \cap \partial V$ with $x_{n}^{*}>-a$. It follows from the convexity of $V$ that $B\left(x, 2 \delta_{D}(x)\right) \backslash D$ contains an open ball of radius $\delta_{D}(x) / 2$ touching $B\left(x, \delta_{D}(x)\right)$ at $x^{*}$. See Figure 7 . Hence Lemma 9.1 yields

$$
{ }^{B\left(x, 4 A t^{1 / \alpha}\right)} \widehat{\mathbf{R}}_{1}^{B\left(x, 2 A t^{1 / \alpha}\right) \backslash D}(x) \geq \eta_{1}
$$

for some $\eta_{1}>0$, We claim that if $r$ satisfies (9.4), then $r s \geq A>0$ as $s \rightarrow \infty$. Suppose, to the contrary, $r=o(1 / s)$. Then we would have

$$
r^{2} s \varphi\left(A_{10} r s\right)=o\left(s^{-2}\right) s \varphi(o(1))=o\left(s^{-1}\right) \rightarrow 0 \quad \text { as } s \rightarrow \infty,
$$

a contradiction to (9.4). The claim implies that if $t>0$ is small, and if $r$ satisfies (9.4), then

$$
r \geq \frac{A}{s}=\frac{A}{\log (1 / t)} \geq 2 A t^{1 / \alpha},
$$

so that ${ }^{B(x, 2 r)} \widehat{\mathbf{R}}_{1}^{B(x, r) \backslash D}(x) \geq \eta_{1}$ by (9.6). That is, if ${ }^{B(x, 2 r)} \widehat{\mathbf{R}}_{1}^{B(x, r) \backslash D}(x)<\eta_{1}$, then $g(x) \geq t$. Letting $\eta \leq \eta_{1}$, we obtain the lemma in case $x_{n}>-a / 2$.

Second, consider the case when $x_{n} \leq-a / 2$. Since ${ }^{B(x, 2 r)} \widehat{\mathbf{R}}_{1}^{B(x, r) \backslash D}(x)$ is increasing in $r$, we may assume $0<r<a / 4$. Let $1<\tau \leq 2, \rho=r / 2$ and $0<\eta \leq \eta_{0}$, with $\eta_{0}$ as in Lemma 9.3. The lemma says that $\left|x^{\prime}\right| \leq R-\rho$, and hence $\left\{B(x, \rho), B\left(x_{1}, \rho\right), \ldots, B\left(x_{J}, \rho\right)\right\}$ is a Harnack simple chain satisfying (9.3) as was observed before Lemma 9.3. Applying Theorem 9.2, we get $g(x) \geq g\left(x_{J}\right) \exp (-A J)$, 


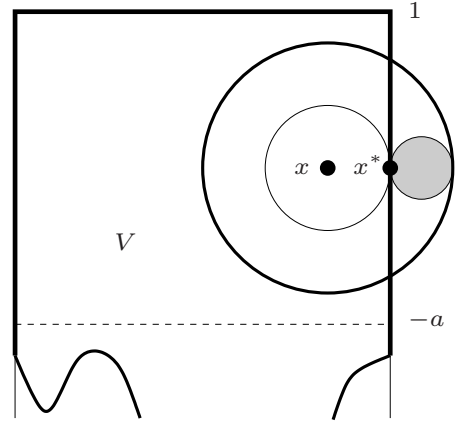

Figure 7. $B\left(x, \delta_{D}(x)\right)$ touches $\partial D$ at $x^{*} \in$ $\partial D \cap \partial V$.

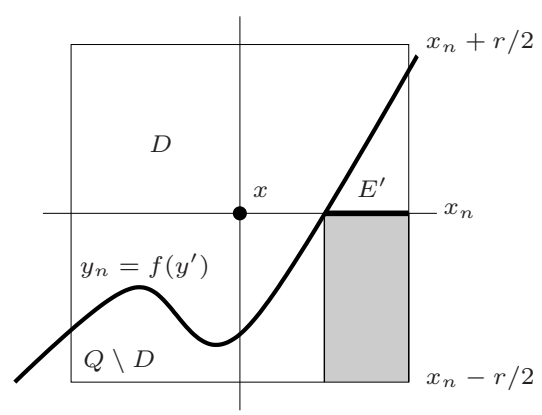

Figure 8. $|Q \backslash D| \geq \frac{r}{2} H_{n-1}\left(E^{\prime}\right)$.

provided $\eta>0$ is sufficiently small. In view of (9.3) and (9.5) with $x_{J}$ in place of $x$, we have

$$
g(x) \geq g\left(x_{J}\right) \exp (-A J) \geq A r^{\alpha} \exp \left(-A \frac{\left|x_{n}\right|}{r}\right) \geq \exp \left(-\frac{\left|x_{n}\right|}{A_{10} r}\right),
$$

where $A_{10}>0$ is taken so small that $A r^{\alpha}$ is absorbed in the last term. This is possible since $\left|x_{n}\right| \geq a / 2$.

Let $Q=B^{\prime}\left(x^{\prime}, r / 2\right) \times\left(x_{n}-r / 2, x_{n}+r / 2\right)$ be a cylinder. Since $Q \subset B(x, r)$, it follows from Lemma 9.1 and ${ }^{B(x, 2 r)} \widehat{\mathbf{R}}_{1}^{B(x, r) \backslash D}(x)<\eta$ that $|Q \backslash D| \leq A \eta|B(x, r)|$. Let $E^{\prime}=\left\{y^{\prime} \in B^{\prime}\left(x^{\prime}, r / 2\right): f\left(y^{\prime}\right) \geq x_{n}\right\}$. Then a line segment $\left\{y^{\prime}\right\} \times\left(x_{n}-r / 2, x_{n}\right)$ of length $r / 2$ lies outside $D$ for $y^{\prime} \in E^{\prime}$. See Figure 8. Hence

$$
\begin{aligned}
|Q \backslash D| & =\int_{B^{\prime}\left(x^{\prime}, r / 2\right)} d y^{\prime} \int_{x_{n}-r / 2}^{x_{n}+r / 2} \chi_{Q \backslash D}(y) d y_{n} \\
& \geq \int_{E^{\prime}} d y^{\prime} \int_{x_{n}-r / 2}^{x_{n}} \chi_{Q \backslash D}(y) d y_{n} \geq \frac{r}{2} H_{n-1}\left(E^{\prime}\right),
\end{aligned}
$$

where $H_{n-1}\left(E^{\prime}\right)$ stands for the $(n-1)$-dimensional Hausdorff measure of $E^{\prime}$. Therefore

$$
\frac{r}{2} H_{n-1}\left(E^{\prime}\right) \leq A \eta r^{n}
$$

so that $H_{n-1}\left(E^{\prime}\right) \leq \frac{1}{2} H_{n-1}\left(B^{\prime}\left(x^{\prime}, r / 2\right)\right)$, provided $\eta$ is sufficiently small. Hence $H_{n-1}\left(B^{\prime}\left(x^{\prime}, r / 2\right) \backslash E^{\prime}\right) \geq \frac{1}{2} H_{n-1}\left(B^{\prime}\left(x^{\prime}, r / 2\right)\right)$. Since $f\left(y^{\prime}\right)<x_{n}$ for $y^{\prime} \in B^{\prime}\left(x^{\prime}, r / 2\right) \backslash E^{\prime}$, it follows that

$\infty>\int_{B^{\prime}(0, R)} \Phi\left(\left|f\left(y^{\prime}\right)\right|\right) d y^{\prime} \geq \int_{B^{\prime}\left(x^{\prime}, r / 2\right) \backslash E^{\prime}} \Phi\left(\left|x_{n}\right|\right) d y^{\prime} \geq 2^{-1} H_{n-1}\left(B^{\prime}\left(x^{\prime}, r / 2\right)\right) \Phi\left(\left|x_{n}\right|\right)$.

Rewriting $\Phi$ by $\varphi$, we obtain

$$
r\left|x_{n}\right| \varphi\left(\left|x_{n}\right|\right) \leq A .
$$


Now let $A_{11}=2 A / A_{10}$ with $A$ being the constant in the right-hand side. If $\left|x_{n}\right| \geq A_{10} r s$, then we would have

$$
A_{10} r^{2} s \varphi\left(A_{10} r s\right) \leq \frac{A_{10} A_{11}}{2}
$$

and hence $r^{2} s \varphi\left(A_{10} r s\right) \leq A_{11} / 2$. This contradicts (9.4). Hence $\left|x_{n}\right|<A_{10} r s$, so that by $(9.7)$

$$
g(x) \geq \exp \left(-\frac{A_{10} r s}{A_{10} r}\right)=\exp (-s)=\exp (-\log (1 / t))=t .
$$

This completes the proof.

Lemma 9.6. Let $\varphi, \Phi, a, f, D$, and $\eta$ be as in Lemma 9.4. If $t>0$ is small, then

$$
w_{\eta}(g<t) \leq A r
$$

where $r$ satisfies (9.4) with $s=\log (1 / t)$.

Proof. Define modified capacitary width by

$$
w_{\eta}^{*}(U)=\inf \left\{r>0:{ }^{B(x, 2 r)} \widehat{\mathbf{R}}_{1}^{B(x, r) \backslash U}(x) \geq \eta \quad \text { for all } x \in U\right\}
$$

for an open set $U$. Lemma 9.4 can be reformulated as $w_{\eta}^{*}(\{x \in D: g(x)<t\}) \leq r$. It is known that $w_{\eta}$ and $w_{\eta}^{*}$ are comparable (Proposition 7.4 in [5]). The lemma follows.

Proof of Theorem 1.26. By the change of variable $s=\log (1 / t)$ we see that $(1.8)$ becomes

$$
\int_{0}^{\infty} w_{\eta}(g<t(s))^{2} d s<\infty .
$$

By Theorem 1.9, it is sufficient to show the above inequality. Lemma 9.6 says that if $s=\log (1 / t)$ is sufficiently large, say $s \geq s_{0}$, then

$$
w_{\eta}(g<t(s)) \leq A \rho(s) \text { with } \rho(s)=\inf \{r>0: r \text { satisfies }(9.4)\} .
$$

Let $1 / 2<\kappa<1$. We split the interval $\left[s_{0}, \infty\right)$ into $I=\left\{s \geq s_{0}: \rho(s) \leq s^{-\kappa}\right\}$ and $I I=\left\{s \geq s_{0}: \rho(s)>s^{-\kappa}\right\}$. Then

$$
\int_{I} \rho(s)^{2} d s \leq \int_{s_{0}}^{\infty} s^{-2 \kappa} d s<\infty .
$$

Suppose $s \in I I$. Take $r \in\left(s^{-\kappa}, \rho(s)\right)$. By definition, this $r$ does not satisfy $(9.4)$, so that, by the monotonicity of $\varphi$,

$$
r^{2} s \varphi\left(A_{10} s^{1-\kappa}\right) \leq r^{2} s \varphi\left(A_{10} r s\right)<A_{11} .
$$


Letting $r \rightarrow \rho(s)$, we obtain

$$
\rho(s)^{2} \leq \frac{A_{11}}{s \varphi\left(A_{10} s^{1-\kappa}\right)} .
$$

By the change of variable $A_{10} s^{1-\kappa}=\sigma$ we have

$$
\begin{aligned}
\int_{I I} \rho(s)^{2} d s & \leq \int_{s_{0}}^{\infty} \frac{A_{11} d s}{s \varphi\left(A_{10} s^{1-\kappa}\right)}=\frac{A_{11}}{1-\kappa} \int_{\sigma_{0}}^{\infty} \frac{d \sigma}{\sigma \varphi(\sigma)} \\
& =\frac{A_{11}}{1-\kappa} \int_{\sigma_{0}}^{\infty} \Phi(\sigma)^{1 /(1-n)} d \sigma<\infty
\end{aligned}
$$

where $\sigma_{0}=A_{10} s_{0}^{1-\kappa}$ by (1.23). Thus we obtain (9.8). The proof is complete.

If $f \in L^{p}\left(B^{\prime}(0, R)\right)$ with $p>n-1$, then the constant $C_{t}$ in $(1.2)$ can be easily estimated.

Proposition 9.7. If $f \in L^{p}\left(B^{\prime}(0, R)\right)$ with $p>n-1$, then $C_{t}$ in (1.2) for $D_{f}$ has an estimate $\log \left(C_{t}\right)=O\left(t^{(n+p-1) /(n-p-1)}\right)$ as $t \rightarrow 0$.

Proof. Since $t^{p}=\Phi(t)=(t \varphi(t))^{n-1}$, it follows that $\varphi(t)=t^{(p-n+1) /(n-1)}$. Then $(9.4)$ can be easily solved, and we can take $r=A s^{-p /(n+p-1)}$. Hence $w_{\eta}(\log (1 / g)>s)$ $\leq A s^{-p /(n+p-1)}$. Let $0<m<1$ and put $M=\log (1 / m)$. Then

$$
\int_{M}^{\infty} w_{\eta}(\log (1 / g)>s)^{2} d s \leq A \int_{M}^{\infty} s^{-2 p /(n+p-1)} d s=\frac{A(n+p-1)}{n-p-1} M^{(n-p-1) /(n+p-1)} .
$$

Hence we can choose $\log (1 / m)=A t^{(n+p-1) /(n-p-1)}$ in $(1.10)$, so that $\log \left(C_{t}\right) \leq$ $\log \left(A_{2} / m^{2+\varepsilon}\right)+A \log (1 / t) \leq A t^{(n+p-1) /(n-p-1)}$.

Remark 9.8. Observe that $-\infty<(n+p-1) /(n-p-1) \leq-1$ for $n-1<p \leq \infty$. So, we do not have $\lim _{t \rightarrow 0} t \log \left(C_{t}\right)=0$ in this setting.

\section{Pasting domains and $\boldsymbol{L}^{\Phi}$-domains}

Let us give a generalization of Theorem 1.26 by pasting finitely many domains. In view of Proposition 3.17 and Remark 4.4, space localization is not so obvious. So, we have to clarify the meaning of pasting.

Definition 10.1. We say that two domains $D_{1}$ and $D_{2}$ are well-pasted if there are two bounded Lipschitz domains $\Omega$ and $\Omega^{*}$ with the following properties: let $D=D_{1} \cup D_{2}$.

(i) $D_{1} \cap D_{2} \subset \Omega \subset \Omega^{*} \subset D$.

(ii) $D \cap \partial \Omega \subset \Omega^{*}$.

(iii) $\operatorname{dist}\left(D \cap \partial \Omega,\left(D_{1} \cap D_{2}\right) \cup\left(D \cap \partial \Omega^{*}\right)\right)>0$.

See Figure 9. 


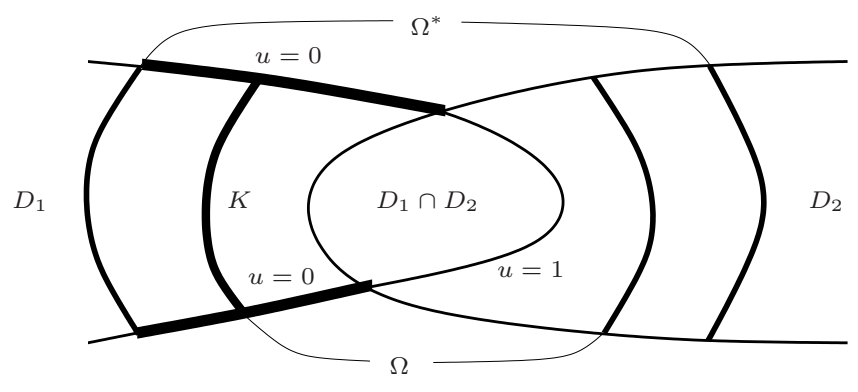

Figure 9. Well-pasted domains.

Lemma 10.2. Assume that two domains $D_{1}$ and $D_{2}$ are well-pasted. Let $D=$ $D_{1} \cup D_{2}, x_{0} \in D_{1} \cap D_{2}$ and let $g_{j}=G_{D_{j}}\left(\cdot, x_{0}\right)$ and $g=G_{D}\left(\cdot, x_{0}\right)$. Suppose, for each $t>0$,

$$
P\left(t, x, D_{j}\right) \leq C_{t}^{j} g_{j}(x) \quad \text { for } x \in D_{j}
$$

holds with $j=1,2$, where $C_{t}^{j}$ is a positive constant depending on $t$ and $D_{j}$. Then for each $t>0$,

$$
P(t, x, D) \leq C_{t} g(x) \quad \text { for } x \in D \text {. }
$$

Proof. Let $\Omega$ and $\Omega^{*}$ be as in Definition 10.1. Let $t>0$. By the parabolic comparison principle for $(0, \infty) \times D_{1}$ and by assumption, we have

$$
P(t, x, D) \leq P\left(t, x, D_{1}\right)+\omega^{x}\left(D_{2} \cap \partial D_{1}, D_{1}\right) \leq C_{t}^{1} g_{1}(x)+\omega^{x}\left(D_{2} \cap \partial D_{1}, D_{1}\right)
$$

for $x \in D_{1}$. Let $u=\omega^{x}\left(D_{2} \cap \partial D_{1}, D_{1}\right)$ on $\Omega^{*} \cap D_{1}$ and let $u=1$ on $\Omega^{*} \backslash D_{1}$. Then $u$ is a bounded positive superharmonic function on $\Omega^{*}$ such that $u$ is harmonic in $\Omega^{*} \cap D_{1}$ and $u$ vanishes q.e. on $\partial D_{1} \cap \partial \Omega^{*} \subset \partial D_{1} \backslash D_{2}$. Let $0<r<\operatorname{dist}(D \cap \partial \Omega$, $\left.\left(D_{1} \cap D_{2}\right) \cup\left(D \cap \partial \Omega^{*}\right)\right)$. Apply the elliptic boundary Harnack principle to $u$ and $g$ with $K=\bar{D}_{1} \cap \partial \Omega$ and $V=\left\{y \in \mathbb{R}^{n}: \operatorname{dist}(y, K)<r\right\}$. Then we have $\omega^{x}\left(D_{2} \cap \partial D_{1}, D_{1}\right)=u(x) \leq A g(x)$ for $x \in D_{1} \cap \partial \Omega$. By the elliptic comparison principle

$$
\omega^{x}\left(D_{2} \cap \partial D_{1}, D_{1}\right) \leq A g(x) \text { for } x \in D_{1} \backslash \Omega .
$$

See Figure 9. Hence we have

$$
P(t, x, D) \leq\left(C_{t}^{1}+A\right) g(x) \text { for } x \in D_{1} \backslash \Omega \text {. }
$$

Replacing $D_{1}$ by $D_{2}$, we obtain the same inequality with $C_{t}^{2}$ in place of $C_{t}^{1}$ for $x \in D_{2} \backslash \Omega$. Hence we have

$$
P(t, x, D) \leq\left(C_{t}^{1}+C_{t}^{2}+A\right) g(x) \text { for } x \in\left(D_{1} \cup D_{2}\right) \backslash \Omega .
$$

By the parabolic comparison principle for $(0, \infty) \times \Omega^{*}$ we have

$$
P(t, x, D) \leq P\left(t, x, \Omega^{*}\right)+\omega^{x}\left(D \cap \partial \Omega^{*}, \Omega^{*}\right) \quad \text { for } x \in \Omega^{*} .
$$


Since $\Omega^{*}$ is a Lipschitz domain, we have

$$
P\left(t, x, \Omega^{*}\right) \leq C_{t} G_{\Omega^{*}}\left(x, x_{0}\right) \leq C_{t} g(x) \text { for } x \in \Omega^{*} .
$$

Since $\operatorname{dist}\left(D \cap \partial \Omega, D \cap \partial \Omega^{*}\right)>0$, it follows from the elliptic boundary Harnack principle on $\Omega^{*}$ that $\omega^{x}\left(D \cap \partial \Omega^{*}, \Omega^{*}\right) \leq A g(x)$ for $x \in \Omega$. Hence

$$
P(t, x, D) \leq\left(C_{t}+A\right) g(x) \text { for } x \in \Omega,
$$

which, together with (10.1), gives the required inequality with different $C_{t}$.

Proposition 10.3. Assume that the domains $D_{1}$ and $D_{2}$ are well-pasted, and that

$$
\lim _{R \rightarrow \infty} w_{\eta}\left(D_{j} \backslash \bar{B}(0, R)\right)=0 \quad \text { for } j=1,2 .
$$

Let $g_{j}=G_{D_{j}}\left(\cdot, x_{0}\right)$ with $x_{0} \in D_{1} \cap D_{2}$. If

$$
\int_{0}^{1} w_{\eta}\left(\left\{x \in D_{j}: g_{j}(x)<t\right\}\right)^{2} \frac{d t}{t}<\infty \quad \text { for } j=1,2,
$$

then $D=D_{1} \cup D_{2}$ is $I U$.

Proof. Since $P\left(t, x, D_{j}\right) \leq C_{t} g_{j}(x)$ for $x \in D_{j}$ by Lemma 4.3, it follows from Lemma 10.2 that

$$
P(t, x, D) \leq C_{t} g(x) \quad \text { for } x \in D
$$

with $g=G_{D}\left(\cdot, x_{0}\right)$. Hence

$$
p(t, x, y) \leq A_{t} g(x) g(y) \quad \text { for } x, y \in D .
$$

by Lemma 5.1. Since $D_{1}$ and $D_{2}$ are well-pasted, we have (1.6), so that

$$
g(x) \leq A \varphi_{D}(x) \text { for } x \in D \backslash B\left(x_{0}, \delta_{D}\left(x_{0}\right) / 2\right),
$$

by Lemma 5.3 . Since $\varphi_{D} \geq A>0$ on $B\left(x_{0}, \delta_{D}\left(x_{0}\right) / 2\right)$ we have

$$
p(t, x, y) \leq A_{t} \varphi_{D}(x) \varphi_{D}(y) \text { for } x, y \in D .
$$

Hence $D$ is IU by Proposition 2.2.

Definition 10.4. Let $\Phi(t)$ be a nondecreasing positive function of $t>0$. We say that $D$ is an $L^{\Phi}$-domain if there exist finitely many domains $D_{1}, \ldots, D_{N}$ such that

(i) $D=D_{1} \cup \cdots \cup D_{N}$, where $D_{1} \cup \cdots \cup D_{j}$ and $D_{j+1}$ are well-pasted for $j=1, \ldots, N-1$;

(ii) each $D_{j}$ is represented as $D_{f}$ as in Theorem B with $f \in L^{\Phi}\left(B^{\prime}(0, R)\right)$ in a suitable coordinate system.

Combining Theorem 1.26 and Proposition 10.3, we obtain the following.

Theorem 10.5. Assume that $\Phi(t) / t^{n-1}$ is nondecreasing and that (1.23) holds. Then every $L^{\Phi}$-domain is $I U$. 


\section{Characterization of IU for an infinite funnel. Proof of Theorem 1.28}

Without loss of generality, we may assume that $r(0)=1$. For simplicity we let

$$
d_{V}(x)=r\left(-x_{n}\right)-\left|x^{\prime}\right| \text { for } x \in V .
$$

This is the distance between $x^{\prime}$ and the horizontal slice of $\partial V$ at height $x_{n}$.

Lemma 11.1. If $x \in V$ and $x_{n} \leq 0$, then

$$
(L+1)^{-1} d_{V}(x) \leq \delta_{V}(x) \leq d_{V}(x) .
$$

Proof. The second inequality readily follows from the definition of $V$. Let us estimate $|x-y|$ for $y \in \partial V$. We have

$|y-x| \geq \begin{cases}\left|y_{n}-x_{n}\right| \geq 1=r(0) \geq d_{V}(x) & \text { if } y_{n}=1, \\ \left|y_{n}-x_{n}\right| \geq(L+1)^{-1} d_{V}(x) & \text { if } y_{n}<1 \text { and }\left|y_{n}-x_{n}\right| \geq(L+1)^{-1} d_{V}(x), \\ \left|y^{\prime}\right|-\left|x^{\prime}\right| \geq(L+1)^{-1} d_{V}(x) & \text { if } y_{n}<1 \text { and }\left|y_{n}-x_{n}\right| \leq(L+1)^{-1} d_{V}(x) .\end{cases}$

Here, in the third case, we have used $d_{V}(y)=r\left(-y_{n}\right)-\left|y^{\prime}\right|=0$, and

$$
\begin{aligned}
\left|y^{\prime}\right|-\left|x^{\prime}\right| & =r\left(-y_{n}\right)-\left(r\left(-x_{n}\right)-d_{V}(x)\right) \geq d_{V}(x)-\left|r\left(-y_{n}\right)-r\left(-x_{n}\right)\right| \\
& \geq d_{V}(x)-L\left|y_{n}-x_{n}\right| \geq\left(1-\frac{L}{L+1}\right) d_{V}(x) .
\end{aligned}
$$

Taking the infimum of $|y-x|$ for $y \in \partial V$, we obtain $\delta_{V}(x) \geq(L+1)^{-1} d_{V}(x)$.

By $x(t)$ we denote the point $(0,-t)$ for $t \geq 0$. Let $x_{0}=x(0)=(0,0)$. The quasihyperbolic distance between $x(t)$ and $x(T)$ is estimated as follows.

Lemma 11.2. Let $0 \leq t<T$. Then

$$
\frac{1}{L} \log \frac{r(t)}{r(T)} \leq \int_{t}^{T} \frac{d \tau}{r(\tau)} \leq k_{V}(x(t), x(T)) \leq(L+1) \int_{t}^{T} \frac{d \tau}{r(\tau)} .
$$

Proof. By symmetry the quasihyperbolic geodesic between $x(t)$ and $x(T)$ is the line segment connecting these points. By Lemma 11.1 we have

$$
(L+1)^{-1} r(\tau) \leq \delta_{V}(x(\tau)) \leq r(\tau)
$$

Take the reciprocal of the above inequality and integrate with respect to $\tau \in$ $[t, T]$. Then we have the second and third inequalities of the lemma. Since $r(\tau)$ is $L$-Lipschitz, we have $r(\tau) \leq r(T)+L(T-\tau)$ for $\tau \leq T$, so that

$$
\int_{t}^{T} \frac{d \tau}{r(\tau)} \geq \int_{t}^{T} \frac{d \tau}{r(T)+L(T-\tau)}=\frac{1}{L} \log \frac{r(T)+L(T-t)}{r(T)} \geq \frac{1}{L} \log \frac{r(t)}{r(T)} .
$$


Lemma 11.3. Let $t \geq 0$ and let $y \in V$ with $y_{n}=-t$. Then

$$
k_{V}(y, x(t)) \leq(L+1) \log \frac{r(t)}{d_{V}(y)} .
$$

Proof. Let $\gamma$ be the line segment connecting $x(t)$ and $y$. By Lemma 11.1

$$
\begin{aligned}
k_{V}(y, x(t)) & \leq \int_{\gamma} \frac{d s}{\delta_{V}(z(s))} \leq(L+1) \int_{\gamma} \frac{d s}{d_{V}(z(s))} \\
& =(L+1) \int_{0}^{\left|y^{\prime}\right|} \frac{d s}{r(t)-s}=(L+1) \log \frac{r(t)}{r(t)-\left|y^{\prime}\right|} .
\end{aligned}
$$

By definition $r(t)-\left|y^{\prime}\right|=d_{V}(y)$, so that the lemma follows.

Lemma 11.4. Let $T \geq 0$. If $y \in V$ satisfies $-T \leq y_{n} \leq 0$ and $d_{V}(y) \geq r(T)$, then

$$
k_{V}\left(y, x_{0}\right) \leq L^{*} k_{V}\left(x(T), x_{0}\right) \quad \text { with } L^{*}=\max \{L(L+1), 1\} .
$$

Proof. Let $t=-y_{n}$. Lemmas 11.2 and 11.3 give

$$
k_{V}(y, x(t)) \leq(L+1) \log \frac{r(t)}{d_{V}(y)} \leq(L+1) \log \frac{r(t)}{r(T)} \leq L(L+1) k_{V}(x(T), x(t)) .
$$

Hence

$$
\begin{aligned}
k_{V}\left(y, x_{0}\right) & \leq k_{V}(y, x(t))+k_{V}\left(x(t), x_{0}\right) \leq L(L+1) k_{V}(x(T), x(t))+k_{V}\left(x(t), x_{0}\right) \\
& \leq L^{*}\left(k_{V}(x(T), x(t))+k_{V}\left(x(t), x_{0}\right)\right)=L^{*} k_{V}\left(x(T), x_{0}\right),
\end{aligned}
$$

where the last equality holds since the line segment connecting $x_{0}$ and $x(T)$ is a geodesic.

Lemma 11.5. Let $L^{*}=\max \{L(L+1), 1\}$. For $T \geq 0$ we put

$$
s=L^{*}(L+1) \int_{0}^{T} \frac{d \tau}{r(\tau)} .
$$

Then $w_{\eta}\left(k_{V}\left(\cdot, x_{0}\right)>s\right) \leq \max \{A \exp (-\alpha s), A r(T)\}$ with some $A, \alpha, \eta>0$ independent of $T$.

Proof. Let $y \in V$ and $k_{V}\left(y, x_{0}\right)>s$. First suppose $y_{n}>0$. Then

$$
s<k_{V}\left(y, x_{0}\right) \leq A \log \frac{\delta_{V}\left(x_{0}\right)}{\delta_{V}(y)}+A
$$

by the Lipschitz nature of $\left\{y \in V: y_{n}>-1\right\}$. Hence $\delta_{V}(y) \leq A \exp (-\alpha s)$ with some $A, \alpha>0$ independent of $T$. Next suppose $y_{n} \leq 0$. Then

$$
k_{V}\left(y, x_{0}\right)>s=L^{*}(L+1) \int_{0}^{T} \frac{d \tau}{r(\tau)} \geq L^{*} k_{V}\left(x(T), x_{0}\right)
$$


by Lemma 11.2 with $t=0$. Hence, either $y_{n}<-T$ or $d_{V}(y)<r(T)$ holds by Lemma 11.4. In the first case $r(T) \geq r\left(-y_{n}\right) \geq d_{V}(y) \geq \delta_{V}(y)$. So, in any case $\delta_{V}(y) \leq r(T)$. Therefore,

$$
\left\{y \in V: k_{V}\left(y, x_{0}\right)>s\right\} \subset\left\{y \in V: \delta_{V}(y) \leq \max \{A \exp (-\alpha s), r(T)\}\right\} .
$$

Since $V$ satisfies the CDC, the required estimate follows from Lemma 3.13.

The next lemma shows that, in Theorem 1.28, (iii) $\Longrightarrow$ (iv).

Lemma 11.6. If $\int_{0}^{\infty} r(t) d t<\infty$, then $\int_{0}^{\infty} w_{\eta}\left(k_{V}\left(\cdot, x_{0}\right)>s\right)^{2} d s<\infty$.

Proof. In view of Lemma 11.5, we have

$$
\begin{aligned}
\int_{0}^{\infty} w_{\eta}\left(k_{V}\left(\cdot, x_{0}\right)>s\right)^{2} d s & \leq \int_{0}^{\infty} \max \{A \exp (-\alpha s), A r(T)\}^{2} d s \\
& \leq A^{2} \int_{0}^{\infty} \exp (-2 \alpha s) d s+A^{2} \int_{0}^{\infty} r(T)^{2} d s
\end{aligned}
$$

where $s$ and $T$ satisfy (11.1). The first integral is convergent; the second integral is equal to

$$
L^{*}(L+1) \int_{0}^{\infty} r(T)^{2} \frac{d T}{r(T)}=L^{*}(L+1) \int_{0}^{\infty} r(T) d T<\infty
$$

by regarding (11.1) as the change of variable.

For (ii) $\Longrightarrow$ (iii) in Theorem 1.28 , we shall employ a scale invariant boundary Harnack principle. For $t \geq 2$ let

$$
V(t)=\left\{x \in V:-t-\frac{r(t)}{2 L}<x_{n}<-t+\frac{r(t)}{2 L}\right\} .
$$

We observe that

$$
B^{\prime}\left(0, \frac{r(t)}{2}\right) \times\left(t-\frac{r(t)}{2 L}, t+\frac{r(t)}{2 L}\right) \subset V(t) \subset B^{\prime}\left(0, \frac{3 r(t)}{2}\right) \times\left(t-\frac{r(t)}{2 L}, t+\frac{r(t)}{2 L}\right),
$$

and that $V(t)$ is a Lipschitz domain with uniformly bounded Lipschitz nature. We have the following scale invariant boundary Harnack principle.

Lemma 11.7. There exists a constant $A_{12}>1$ independent of $t \geq 0$ with the following property: Let $S(t)=\left\{x \in V: x_{n}=-t\right\}$. If $u$ and $v$ are positive harmonic functions in $V(t) \backslash\{x(t)\}$, and if $u=v=0$ on $\left\{x \in \partial V:-t-r(t) /(2 L)<x_{n}<\right.$ $-t+r(t) /(2 L)\}$, then

$$
\frac{u(x) / u(y)}{v(x) / v(y)} \leq A_{12} \quad \text { for } x, y \in S\left(t+\frac{r(t)}{4 L}\right) .
$$

See Figure 10. 


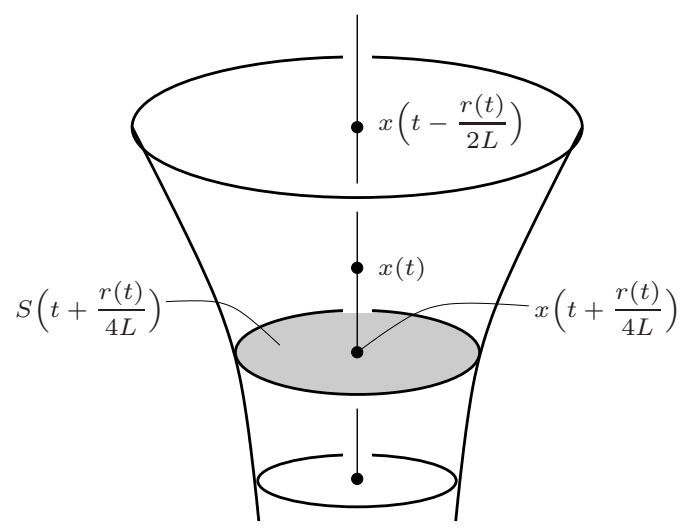

FiguRE 10. Scale invariant boundary Harnack principle for an infinite funnel.

The next lemma is inspired by Hansen [22].

Lemma 11.8. There exists a positive harmonic function $h$ on $V$ vanishing on the boundary $\partial V$ such that $g(x(t)) h(x(t)) \approx r(t)^{2-n}$ for $t>2$.

Proof. In this proof, all constants will be independent of $t$. Let $t>2$. We claim

$$
G\left(x(t), x\left(t+\frac{r(t)}{4 L}\right)\right) \approx r(t)^{2-n} .
$$

Observe that

$$
\begin{aligned}
\left.\mid x(t)-x\left(t+\frac{r(t)}{4 L}\right)\right) \mid & =\frac{r(t)}{4 L} \approx \operatorname{diam} V(t) \\
& \left.\approx \operatorname{dist}(x(t), \partial V(t)) \approx \operatorname{dist}\left(x\left(t+\frac{r(t)}{4 L}\right)\right), \partial V(t)\right),
\end{aligned}
$$

so that

$$
G\left(x(t), x\left(t+\frac{r(t)}{4 L}\right)\right) \geq G_{V(t)}\left(x(t), x\left(t+\frac{r(t)}{4 L}\right)\right) \approx r(t)^{2-n}
$$

by uniform ellipticity. Thus the lower estimate of the claim follows. We have the upper estimate by comparing $G$ and the Green function for the whole space if $n \geq 3$, or the Green function of the complement of a closed disk of radius $r(t)$ lying outside $V$ if $n=2$.

Apply Lemma 11.7 to $u=r(t)^{n-2} G(x(t), \cdot)$ and $v=g / g(x(t))$. Since

$$
u\left(x\left(t+\frac{r(t)}{4 L}\right)\right) \approx v\left(x\left(t+\frac{r(t)}{4 L}\right)\right) \approx 1
$$

by the claim and the Harnack inequality, it follows that

$$
r(t)^{n-2} G(x(t), y) \approx g(y) / g(x(t)),
$$


or equivalently

$$
g(x(t)) \cdot \frac{G(x(t), y)}{g(y)} \approx r(t)^{2-n} \quad \text { for all } y \in S\left(t+\frac{r(t)}{4 L}\right) .
$$

By the maximum principle the same comparison holds for $y_{n} \leq-t-r(t) /(4 L)$. Letting $y_{n} \rightarrow-\infty$, and taking a subsequence, if necessary, we obtain a Martin kernel

$$
h(x)=\lim _{y_{n} \rightarrow-\infty} \frac{G(x, y)}{g(y)} .
$$

such that $g(x(t)) h(x(t)) \approx r(t)^{2-n}$ and $h\left(x_{0}\right)=1$. Thus this $h$ is a required positive harmonic function.

Remark 11.9. We can show that the above limit exists and that it is the unique Martin kernel corresponding to $y_{n} \rightarrow-\infty$ with reference point at $x_{0}$. In fact, if $H$ is another Martin kernel corresponding to $y_{n} \rightarrow-\infty$ with reference point at $x_{0}$, then $H$ enjoys the same estimate as $h$, so that $H \approx h$ on $V$ by Lemma 11.7. Now we can easily show that $H=h$ on $V$ by Kemper's technique (see Theorem 1.1 in [23] and Proof of Theorem 3 in [2]). Note that Kemper's proof of the boundary Harnack principle for a Lipschitz domain had a gap. However his technique for the uniqueness of Martin kernel was correct.

The next lemma implies that, in Theorem 1.28 , (iv) $\Longleftrightarrow(\mathrm{v})$.

Lemma 11.10. There are positive constants $A, \alpha, \beta$ such that

$$
A^{-1} \exp \left(-\alpha k_{V}\left(x, x_{0}\right)\right) \leq g(x) \leq A \exp \left(-\beta k_{V}\left(x, x_{0}\right)\right)
$$

for $x \in V \backslash B\left(x_{0}, \delta_{V}\left(x_{0}\right) / 2\right)$.

The proof is elementary but is rather involved. So, we postpone the proof of the lemma and complete the proof of Theorem 1.28.

Proof of Theorem 1.28. It is well known that IU implies the validity of the Cranston-McConnell inequality for arbitrary domain (Proposition 2.3). Hence (i) $\Longrightarrow$ (ii) holds. In Lemma 11.6 we have shown (iii) $\Longrightarrow$ (iv). Corollary 1.15 asserts that (iv) $\Longrightarrow$ (i). Lemma 11.10 gives (iv) $\Longleftrightarrow$ (v). Let us complete the proof by showing (ii) $\Longrightarrow$ (iii). Let $h$ be the positive harmonic function in Lemma 11.8. Then the Harnack inequality implies $g(x) h(x) \approx r\left(-x_{n}\right)^{2-n}$ for $x \in V_{0}=\left\{x=\left(x^{\prime}, x_{n}\right)\right.$ : $\left.x_{n}<-2, d_{V}(x) \leq 2^{-1} r\left(-x_{n}\right)\right\}$. Hence

$$
\begin{aligned}
\int_{V} G\left(x, x_{0}\right) h(x) d x & \geq \int_{V_{0}} g(x) h(x) d x \approx \int_{V_{0}} r\left(-x_{n}\right)^{2-n} d x \\
& \approx \int_{2}^{\infty} r(t)^{2-n} r(t)^{n-1} d t=\int_{2}^{\infty} r(t) d t .
\end{aligned}
$$

If (ii) holds, then the first integral is convergent, and so is the last integral. Thus (iii) holds. 
Proof of Corollary 1.30. Let $r(t)=(t+3)^{-1}$. Let $y \in V$. First suppose that $t=-y_{n} \geq 0$. Observe that $\delta_{V}(y) \leq(t+3)^{-1}$, or equivalently $t+3 \leq \delta_{V}(y)^{-1}$. Lemmas 11.1, 11.2 and 11.3 yield

$$
\begin{aligned}
k_{V}\left(y, x_{0}\right) & \leq k_{V}(y, x(t))+k_{V}\left(x(t), x_{0}\right) \leq(L+1)\left\{\log \frac{r(t)}{d_{V}(y)}+\int_{0}^{t} \frac{d \tau}{r(\tau)}\right\} \\
& \leq(L+1)\left\{\log \frac{1}{(t+3) \delta_{V}(y)}+\frac{(t+3)^{2}}{2}\right\} \leq \frac{A}{\delta_{V}(y)^{2}}+A .
\end{aligned}
$$

Next suppose $y_{n} \geq 0$. Since $\left\{y \in V: y_{n}>-1\right\}$ is a Lipschitz domain, it follows that

$$
k_{V}\left(y, x_{0}\right) \leq A \log \frac{A}{\delta_{V}(y)}+A \leq \frac{A}{\delta_{V}(y)^{2}}+A .
$$

Thus $V$ satisfies the $\mathrm{QHB}(2)$. Obviously, $\int_{0}^{\infty} r(t) d t=\infty$, so that $V$ is not IU by Theorem 1.28.

Proof of Corollary 1.31. Let $\varphi(s)=s \log ^{\alpha} s$ with $(n-1)^{-1}<\alpha \leq 1$. Then $\varphi^{\prime}(s)=$ $(\log s+\alpha) \log ^{\alpha-1} s>0$ for $s>1$. Hence $r(t)=(t+3)^{-1} \log ^{-\alpha}(t+3)=1 / \varphi(t+3)$ is strictly decreasing for $t>-2$. Let $f\left(x^{\prime}\right)=-r^{-1}\left(\left|x^{\prime}\right|\right)$ for $\left|x^{\prime}\right|<r(-1)$. Then

$$
V=\left\{\left(x^{\prime}, x_{n}\right): x^{\prime} \in B^{\prime}(0, r(-1)), x_{n}>f\left(x^{\prime}\right)\right\} .
$$

Observe that $f\left(x^{\prime}\right)<0$ for $\left|x^{\prime}\right|<r(0)$ and

$$
\begin{aligned}
\int_{B^{\prime}(0, r(0))}\left|f\left(x^{\prime}\right)\right|^{n-1} d x^{\prime} & =\int_{0}^{\infty} H_{n-1}\left(\left\{x^{\prime} \in B^{\prime}(0, r(0)):\left|f\left(x^{\prime}\right)\right|>t\right\}\right) d t^{n-1} \\
& =\int_{0}^{\infty} H_{n-1}\left(B^{\prime}(0, r(t))\right) d t^{n-1}=\int_{0}^{\infty} b_{n-1} r(t)^{n-1} d t^{n-1},
\end{aligned}
$$

where $b_{n-1}$ is the $(n-1)$-dimensional volume of a unit ball in $\mathbb{R}^{n-1}$. The last integral is convergent by $\alpha>(n-1)^{-1}$. Since $0 \leq f\left(x^{\prime}\right) \leq 1$ for $r(0) \leq\left|x^{\prime}\right| \leq r(-1)$, it follows that $V$ is an $L^{n-1}$-domain. On the other hand, $\int_{0}^{\infty} r(t) d t=\infty$ by $\alpha \leq 1$, so that $V$ is not IU by Theorem 1.28 .

Proof of Lemma 11.10. The first inequality of (11.2) is nothing but the first inequality of (1.14). The second inequality is non-trivial. The argument of the exponential function of the right-hand side of (11.2) is a negative multiple of $k_{D}\left(x, x_{0}\right)$, whereas that of (1.14) is a positive multiple. Since $\left\{x=\left(x^{\prime}, x_{n}\right) \in V: x_{n}>-2\right\}$ is a Lipschitz domain, we can easily show (11.2) for $x_{n} \geq-1$. So let us show (11.2) for $x_{n} \leq-1$. For $t \geq 1$ we let $U(t)=\left\{x \in V: x_{n}<-t\right\}, S(t)=\left\{x \in V: x_{n}=-t\right\}$ and

$$
M(t)=\sup _{x \in U(t)} g(x) .
$$

By the maximum principle $M(t)=\sup _{x \in S(t)} g(x)$ and $M(t)$ is decreasing for $t \geq 1$. We claim that there exists a constant $\alpha_{0} \in(0,1)$ such that

$$
M(t+\kappa r(t)) \leq \alpha_{0} M(t) \text { for } t \geq 1,
$$


where $\kappa=(2 L)^{-1}$, with $L$ being the Lipschitz constant of $r(t)$. To see this, let us consider the harmonic measure $v_{r}(x)=\omega^{x}\left(B^{\prime}(0, r) \times\{-t\}, B^{\prime}(0, r) \times(-\infty,-t)\right)$ over the half cylinder $B^{\prime}(0, r) \times(-\infty,-t)$. By translation and dilation we have

$$
v_{r}(x) \leq \alpha_{0} \quad \text { for } x \in B^{\prime}(0, r) \times(-\infty,-t-\kappa r)
$$

with $0<\alpha_{0}<1$ depending only on $\kappa, c_{\mathcal{L}}$ and $n$. Since $U(t) \subset B^{\prime}(0, r(t)) \times$ $(-\infty,-t)$, it follows from the maximum principle that

$$
g(x) \leq M(t) \omega^{x}(S(t), U(t)) \leq M(t) v_{r(t)}(x) \text { for } x \in U(t) .
$$

Taking the supremum for $x \in U(t+\kappa r(t))$, we obtain (11.3).

It follows from (11.3) that $M(t)$ decays exponentially. We claim that

$$
M(t) \leq A \exp \left(-\beta_{0} \int_{0}^{t} \frac{d \tau}{r(\tau)}\right) \quad \text { for } t \geq 1 \text { with } \beta_{0}=L \log \frac{1}{\alpha_{0}} .
$$

To see this, let

$$
\psi(t)=\exp \left(-\beta_{0} \int_{0}^{t} \frac{d \tau}{r(\tau)}\right) \text { and } \varphi(t)=\frac{M(t)}{\psi(t)} .
$$

It suffices to show that $\varphi(t)$ is bounded for $t \geq 1$. Since

$$
\int_{t}^{t+\kappa r(t)} \frac{d \tau}{r(\tau)} \leq \frac{\kappa r(t)}{r(t+\kappa r(t))} \leq \frac{\kappa r(t)}{r(t)-L \kappa r(t)}=\frac{\kappa}{1-L \kappa}=\frac{1}{L},
$$

it follows that

$$
\frac{\psi(t+\kappa r(t))}{\psi(t)}=\exp \left(-\beta_{0} \int_{t}^{t+\kappa r(t)} \frac{d \tau}{r(\tau)}\right) \geq \exp \left(-\frac{\beta_{0}}{L}\right)=\alpha_{0},
$$

so that $\psi(t+\kappa r(t)) \geq \alpha_{0} \psi(t)$. This, together with (11.3), implies that

$$
\varphi(t+\kappa r(t))=\frac{M(t+\kappa r(t))}{\psi(t+\kappa r(t))} \leq \frac{\alpha_{0} M(t)}{\alpha_{0} \psi(t)}=\varphi(t) .
$$

We complete the proof of (11.4) by showing that

$$
\varphi(t) \leq A_{13} \text { for } t \geq 1,
$$

where $A_{13}=\sup _{1 \leq \tau \leq 1+\kappa r(1)} \varphi(\tau)$. In fact, let

$$
t^{*}=\sup \left\{t \geq 1: \varphi(\tau) \leq A_{13} \text { for } 1 \leq \tau \leq t\right\} .
$$

By definition $t^{*} \geq 1+\kappa r(1)$. It is sufficient to show that $t^{*}=\infty$. Suppose, to the contrary, $t^{*}<\infty$. Since $t \mapsto t+\kappa r(t)$ is a continuous mapping, it follows from the 
mean value theorem that for each $\tau \in\left[1+\kappa r(1), t^{*}+\kappa r\left(t^{*}\right)\right]$ we find $t_{1} \in\left[1, t^{*}\right]$ such that $t_{1}+\kappa r\left(t_{1}\right)=\tau$. Hence (11.5) with $t_{1}$ in place of $t$ gives

$$
\varphi(\tau)=\varphi\left(t_{1}+\kappa r\left(t_{1}\right)\right) \leq \varphi\left(t_{1}\right) \leq A_{13},
$$

so that $\varphi(\tau) \leq A_{13}$ for $1 \leq \tau \leq t^{*}+\kappa r\left(t^{*}\right)$, which contradicts the maximality of $t^{*}$. Hence (11.6) and so (11.4) follow. In view of Lemma 11.2, we have

$$
g(x(t)) \leq M(t) \leq A \exp \left(-\frac{\beta_{0}}{L+1} k_{V}\left(x(t), x_{0}\right)\right) \quad \text { for } t \geq 1 .
$$

Next we claim that

$$
g(y) \leq A\left(\frac{\delta_{V}(y)}{\delta_{V}(x(t))}\right)^{\beta_{1}} g(x(t)) \quad \text { for } y \in S(t)
$$

with some $\beta_{1}>0$. Recall that

$$
V(t)=\left\{x \in V:-t-\frac{r(t)}{2 L}<x_{n}<-t+\frac{r(t)}{2 L}\right\}
$$

(see the discussion before Lemma 11.7). Let $B=B\left(x(t), \delta_{V(t)}(x(t)) / 4\right)$ and $B^{*}=$ $B\left(x(t), \delta_{V(t)}(x(t)) / 2\right)$. By the Harnack inequality, $g \approx g(x(t))$ on $B^{*}$, so it suffices to show (11.8) for $y \in S(t) \backslash B^{*}$. By the scale invariant boundary Harnack principle (Lemma 11.7, slightly modified), we have

$$
g \leq A g(x(t)) \omega(\partial B, V(t) \backslash \bar{B}) \quad \text { on } S(t) \backslash B^{*} .
$$

Since $V(t)$ satisfies the CDC, we have a strong barrier $s$ such that $\mathcal{L} s+\left(\varepsilon / \delta_{V(t)}^{2}\right) s \leq 0$ and $s(y) \approx \delta_{V(t)}(y)^{\beta_{1}}$ with $\varepsilon>0$ and $\beta_{1}>0$ depending only on $L, c_{\mathcal{L}}$ and $n$ (see Theorem 3 in [6]). In particular, $s / s(x(t)) \approx 1$ on $\bar{B}$, so that the maximum principle implies $\omega(\partial B, V(t) \backslash \bar{B}) \leq A s / s(x(t))$ on $V(t) \backslash \bar{B}$, and hence

$$
g(y) \leq A g(x(t)) \cdot \frac{s(y)}{s(x(t))} \leq A g(x(t))\left(\frac{\delta_{V}(y)}{\delta_{V}(x(t))}\right)^{\beta_{1}} \quad \text { for } y \in S(t) \backslash B^{*},
$$

as $\delta_{V(t)}(y) \approx \delta_{V}(y)$ for $y \in S(t)$. Thus we obtain (11.8).

Now the proof is easy. Since $\delta_{V}(y) \approx d_{V}(y)$ for $y \in S(t)$, it follows from Lemma 11.3 that

$$
\frac{\delta_{V}(y)}{\delta_{V}(x(t))} \approx \frac{d_{V}(y)}{r(t)} \leq \exp \left(-\frac{1}{L+1} k_{V}(y, x(t))\right) .
$$

This, together with (11.7) and (11.8), yields

$$
g(y) \leq A \exp \left(-\frac{\beta_{0}}{L+1} k_{V}\left(x(t), x_{0}\right)-\frac{\beta_{1}}{L+1} k_{V}(y, x(t))\right) \leq A \exp \left(-\beta k_{V}\left(y, x_{0}\right)\right)
$$

for $y \in S(t)$ with $\beta=(L+1)^{-1} \min \left\{\beta_{0}, \beta_{1}\right\}$. Since $t \geq 1$ is arbitrary, we have (11.2) for $x_{n} \leq-1$. The proof is complete. 


\section{Appendix. Proofs of Lemmas D, E and F}

In this appendix we prove Lemmas D, E and F. These lemmas were proved in [1] in the case $\mathcal{L}=\Delta$. For completeness, we provide complete arguments in the $\mathcal{L}$-harmonic context. Throughout this appendix, we explicitly write the prefix, super and subscripts $\mathcal{L}$ in order to clarify the difference between $\mathcal{L}$ and $\Delta$. Let us begin with some uniform estimates for the capacity of balls.

Lemma 12.1. Let $0<t \leq 1$. Define

$$
\kappa(t)=\inf \left\{\frac{\operatorname{Cap}_{B(x, 2 R)}^{\mathcal{L}}(\bar{B}(x, t R))}{\operatorname{Cap}_{B(x, 2 R)}^{\mathcal{L}}(\bar{B}(x, R))}\right\},
$$

where the infimum is taken over all $x \in \mathbb{R}^{n}, R>0$ and uniformly elliptic operators $\mathcal{L}$ satisfying (1.1). Then $\kappa(t)>0$ and $\lim _{t \rightarrow 1} \kappa(t)=1$.

Proof. Let $u$ be the capacitary potential for $\bar{B}(x, t R)$ in $B(x, 2 R)$, i.e.,

$$
\begin{aligned}
& \mathcal{L} u=0 \text { in } B(x, 2 R) \backslash \bar{B}(x, t R), \\
& u=1 \text { on } \bar{B}(x, t R), \\
& u=0 \text { on } \partial B(x, 2 R), \\
& \operatorname{Cap}_{B(x, 2 R)}^{\mathcal{L}}(\bar{B}(x, t R))=Q_{B(x, 2 R)}[u] .
\end{aligned}
$$

Put $v(y)=u(t R y+x)$. Then

$$
\begin{aligned}
\widetilde{\mathcal{L}} v=0 & \text { in } B(0,2 / t) \backslash \bar{B}(0,1), \\
v=1 & \text { on } \bar{B}(0,1), \\
v=0 & \text { on } \partial B(0,2 / t),
\end{aligned}
$$

where $\widetilde{\mathcal{L}}$ is a uniform elliptic operator satisfying (1.1). Let $\Omega=B(0,2) \backslash \bar{B}(0,1)$ and let $s$ be the strong barrier for $\Omega$ with respect to $\widetilde{\mathcal{L}}$, i.e., $\widetilde{\mathcal{L}} s+\left(\varepsilon / \delta_{\Omega}^{2}\right) s \leq 0$ and

$$
A^{-1} \delta_{\Omega}(y)^{\alpha} \leq s(y) \leq A \delta_{\Omega}(y)^{\alpha} \text { for } y \in \Omega,
$$

where $\varepsilon>0, \alpha>0$ and $A \geq 1$ depend only on $c_{\mathcal{L}}$ and $n$. See Theorem 3 in [6]. Let us compare $1-v$ and $s$ on $B(0,3 / 2) \backslash \bar{B}(0,1)$. Since $\delta_{\Omega}(y)=|y|-1$ for $y \in \bar{B}(0,3 / 2) \backslash \bar{B}(0,1)$, it follows from (12.1) that

$$
s(y) \geq A^{-1}\left(\frac{3}{2}-1\right)^{\alpha}=\frac{1}{2^{\alpha} A} \quad \text { for } y \in \partial B(0,3 / 2) .
$$

so that the maximum principle, together with (12.1), yields

$$
1-v(y) \leq 2^{\alpha} A s(y) \leq 2^{\alpha} A^{2} \delta_{\Omega}(y)^{\alpha} \leq 2^{\alpha} A^{2}(|y|-1)^{\alpha} \quad \text { for } y \in \bar{B}(0,3 / 2) \backslash \bar{B}(0,1) .
$$

Rewriting the inequality with $u$, we obtain

$$
u(z) \geq 1-2^{\alpha} A^{2}\left(\frac{|z-x|}{t R}-1\right)^{\alpha} \text { for } z \in \bar{B}\left(x, \frac{3 t R}{2}\right) \backslash \bar{B}(x, t R),
$$


Let

$$
c_{1}=1+\frac{1}{2^{1+1 / \alpha} A^{2 / \alpha}}
$$

Then $1<c_{1}<3 / 2$ and $2^{\alpha} A^{2}\left(c_{1}-1\right)^{\alpha}=1 / 2$. Hence (12.2) yields $u \geq 1 / 2$ on $\bar{B}\left(x, c_{1} t R\right) \backslash \bar{B}(x, t R)$. Since $u=1$ on $\bar{B}(x, t R)$, we have

$$
u \geq \frac{1}{2} \text { on } \bar{B}\left(x, c_{1} t R\right)
$$

which implies that

$\operatorname{Cap}_{B(x, 2 R)}^{\mathcal{L}}\left(\bar{B}\left(x, c_{1} t R\right)\right) \leq Q_{B(x, 2 R)}[2 u]=4 Q_{B(x, 2 R)}[u]=4 \operatorname{Cap}_{B(x, 2 R)}^{\mathcal{L}}(\bar{B}(x, t R))$.

Let $k \geq 1$ be the least integer such that $c_{1}^{k} t \geq 1$. Repeated application of the above inequality gives

$$
\operatorname{Cap}_{B(x, 2 R)}^{\mathcal{L}}(\bar{B}(x, R)) \leq \operatorname{Cap}_{B(x, 2 R)}^{\mathcal{L}}\left(\bar{B}\left(x, c_{1}^{k} t R\right)\right) \leq 4^{k} \operatorname{Cap}_{B(x, 2 R)}^{\mathcal{L}}(\bar{B}(x, t R)) .
$$

This shows $\kappa(t) \geq 4^{-k}$. The first assertion of the lemma follows.

For the second assertion we may assume that $2 / 3 \leq t<1$. Then (12.2) yields

$$
u \geq 1-2^{\alpha} A^{2}\left(\frac{R}{t R}-1\right)^{\alpha}=1-2^{\alpha} A^{2}\left(t^{-1}-1\right)^{\alpha} \quad \text { on } \bar{B}(x, R) \backslash \bar{B}(x, t R) .
$$

In the same way as above, we have

$$
u \geq \beta(t) \quad \text { on } \bar{B}(x, R)
$$

with $\beta(t)=1-3^{\alpha} A^{2}(1-t)^{\alpha}$ since $2 / 3 \leq t<1$. If $t$ is close to 1 , then $\beta(t)>0$, so that

$$
\operatorname{Cap}_{B(x, 2 R)}^{\mathcal{L}}(\bar{B}(x, R)) \leq Q_{B(x, 2 R)}\left[\beta(t)^{-1} u\right]=\beta(t)^{-2} \operatorname{Cap}_{B(x, 2 R)}^{\mathcal{L}}(\bar{B}(x, t R)) .
$$

Hence

$$
\frac{\operatorname{Cap}_{B(x, 2 R)}^{\mathcal{L}}(\bar{B}(x, t R))}{\operatorname{Cap}_{B(x, 2 R)}^{\mathcal{L}}(\bar{B}(x, R))} \geq \beta(t)^{2} \uparrow 1 \quad \text { as } t \rightarrow 1 .
$$

Thus $\lim _{t \rightarrow 1} \kappa(t)=1$. The lemma is proved.

We recall regularized reduced functions ${ }^{U} \widehat{\mathbf{R}}_{u}^{E}$ used in Section 9. Note that regularized reduced functions are taken with respect to $\mathcal{L}$-superharmonic functions. However, we suppress " $\mathcal{L}-"$ to simplify notation.

Proof of Lemma F. Let $\mu_{E}$ and $\nu$ be the capacitary measures of $E$ and $\bar{B}(x, r)$, respectively. Then $\mu_{E}$ is supported on $E, G_{B(x, R)}^{\mathcal{L}} \mu_{E}={ }^{B(x, R)} \widehat{\mathbf{R}}_{1}^{E}$ and $\left\|\mu_{E}\right\|=$ $\operatorname{Cap}_{B(x, R)}^{\mathcal{L}}(E) ; \nu$ is supported on $\partial B(x, r), G_{B(x, R)}^{\mathcal{L}} \nu={ }^{B(x, R)} \widehat{\mathbf{R}}_{1}^{\bar{B}(x, r)}$ and $\|\nu\|=$ $\operatorname{Cap}_{B(x, R)}^{\mathcal{L}}(\bar{B}(x, r))$. In particular, $G_{B(x, R)}^{\mathcal{L}} \nu \leq 1$ on $B(x, R)$ and hence

$$
\begin{aligned}
\operatorname{Cap}_{B(x, R)}^{\mathcal{L}}(E) & \geq \int G_{B(x, R)}^{\mathcal{L}} \nu d \mu_{E}=\int G_{B(x, R)}^{\mathcal{L}} \mu_{E} d \nu=\int B(x, R) \widehat{\mathbf{R}}_{1}^{E} d \nu \\
& \geq \int\left(\inf _{\bar{B}(x, r)} B(x, R) \widehat{\mathbf{R}}_{1}^{E}\right) d \nu=\left(\inf _{\bar{B}(x, r)} B(x, R) \widehat{\mathbf{R}}_{1}^{E}\right) \operatorname{Cap}_{B(x, R)}^{\mathcal{L}}(\bar{B}(x, r)) .
\end{aligned}
$$


Thus the first assertion follows. For the second assertion let $\rho=(r+R) / 2$. We observe from the Harnack inequality that there are $A_{14}, A_{15}>1$ depending only on $n, r / R$ and $c_{\mathcal{L}}$ such that

$$
\begin{aligned}
& A_{14}^{-1} \leq \frac{G_{B(x, R)}^{\mathcal{L}}(z, y)}{G_{B(x, R)}^{\mathcal{L}}(z, x)} \leq A_{14} \quad \text { for } z \in \partial B(x, \rho) \text { and } y \in \bar{B}(x, r), \\
& A_{15}^{-1} \leq{ }^{B(x, R)} \widehat{\mathbf{R}}_{1}^{\bar{B}(x, r)} \leq 1 \quad \text { on } \partial B(x, \rho) .
\end{aligned}
$$

Let $z \in \partial B(x, \rho)$. If $E \subset \bar{B}(x, r)$, then supp $\mu_{E} \subset \bar{B}(x, r)$, so that

$$
\begin{aligned}
{ }^{B(x, R)} \widehat{\mathbf{R}}_{1}^{E}(z) & =\int G_{B(x, R)}^{\mathcal{L}}(z, y) d \mu_{E}(y) \\
& \geq A_{14}^{-1} G_{B(x, R)}^{\mathcal{L}}(z, x)\left\|\mu_{E}\right\|=A_{14}^{-1} G_{B(x, R)}^{\mathcal{L}}(z, x) \operatorname{Cap}_{B(x, R)}^{\mathcal{L}}(E),
\end{aligned}
$$

and

$$
\begin{aligned}
A_{15}^{-1} & \leq{ }^{B(x, R)} \widehat{\mathbf{R}}_{1}^{\bar{B}(x, r)}(z)=\int G_{B(x, R)}^{\mathcal{L}}(z, y) d \nu(y) \leq A_{14} G_{B(x, R)}^{\mathcal{L}}(z, x)\|\nu\| \\
& =A_{14} G_{B(x, R)}^{\mathcal{L}}(z, x) \operatorname{Cap}_{B(x, R)}^{\mathcal{L}}(\bar{B}(x, r)) .
\end{aligned}
$$

Hence

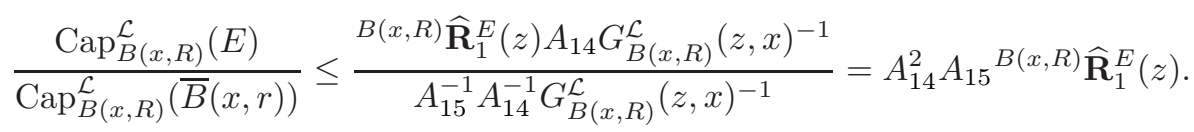

Since $z \in \partial B(x, \rho)$ is arbitrary, the second assertion holds with $A=A_{14}^{2} A_{15}$ by the maximum principle.

It is convenient to restate the above lemma in terms of $\mathcal{L}$-harmonic measure. By $\omega_{\mathcal{L}}^{x}(E, D)$ we denote the $\mathcal{L}$-harmonic measure of $E$ in $D$ evaluated at $x$. We see that if $E$ is a compact subset of $B(x, R)$, then

$$
\omega_{\mathcal{L}}(\partial B(x, R), B(x, R) \backslash E)=1-{ }^{B(x, R)} \widehat{\mathbf{R}}_{1}^{E} \quad \text { on } B(x, R) .
$$

Strictly speaking, $\mathcal{L}$-harmonic measure is extended by 0 on $E$, which coincides q.e. with the right-hand side. Lemma $\mathrm{F}$ reads as follows.

Lemma 12.2. Let $0<r<R$. If $E$ is a compact subset of $B(x, R)$, then

$$
1-\frac{\operatorname{Cap}_{B(x, R)}^{\mathcal{L}}(E)}{\operatorname{Cap}_{B(x, R)}^{\mathcal{L}}(\bar{B}(x, r))} \leq \sup _{\bar{B}(x, r)} \omega_{\mathcal{L}}(\partial B(x, R), B(x, R) \backslash E) .
$$

Moreover, if $E \subset \bar{B}(x, r)$, then

$$
\sup _{\bar{B}(x, r)} \omega_{\mathcal{L}}(\partial B(x, R), B(x, R) \backslash E) \leq 1-A^{-1} \frac{\operatorname{Cap}_{B(x, R)}^{\mathcal{L}}(E)}{\operatorname{Cap}_{B(x, R)}^{\mathcal{L}}(\bar{B}(x, r))},
$$


where $A>1$ depends only on $n, c_{\mathcal{L}}$ and $r / R$; in particular,

$$
\sup _{\bar{B}(x, r)} \omega_{\mathcal{L}}(\partial B(x, 2 r), B(x, 2 r) \backslash E) \leq 1-A_{16}^{-1} \frac{\operatorname{Cap}_{B(x, 2 r)}^{\mathcal{L}}(E)}{\operatorname{Cap}_{B(x, 2 r)}^{\mathcal{L}}(\bar{B}(x, r))},
$$

where $A_{16}>1$ depends only on $n$ and $c_{\mathcal{L}}$.

Let us define capacitary width with respect to $\mathcal{L}$. See Definition 1.2.

Definition 12.3. Let $0<\eta<1$. For an open set $D$ we define the capacitary width $w_{\eta}^{\mathcal{L}}(D)$ with respect to $\mathcal{L}$ by

$$
w_{\eta}^{\mathcal{L}}(D)=\inf \left\{r>0: \frac{\operatorname{Cap}_{B(x, 2 r)}^{\mathcal{L}}(\bar{B}(x, r) \backslash D)}{\operatorname{Cap}_{B(x, 2 r)}^{\mathcal{L}}(\bar{B}(x, r))} \geq \eta \quad \text { for all } x \in D\right\} .
$$

If $\mathcal{L}$ is the Laplacian, we simply write $w_{\eta}(D)$.

Remark 12.4. Definitions 1.2 and 12.3 are consistent since $\operatorname{Cap}_{B(x, 2 r)}^{\mathcal{L}}(\bar{B}(x, r))=$ $\operatorname{Cap}_{B(x, 2 r)}^{\mathcal{L}}(B(x, r))$, and since $\operatorname{Cap}_{B(x, 2 r)}^{\mathcal{L}}(B(x, r) \backslash D) \leq \operatorname{Cap}_{B(x, 2 r)}^{\mathcal{L}}(\bar{B}(x, r) \backslash D) \leq$ $\operatorname{Cap}_{B(x, 2 r)}^{\mathcal{L}}\left(B\left(x, r^{\prime}\right) \backslash D\right)$ for $0<r<r^{\prime}$. Definition 12.3 has an advantage that $E=\bar{B}(x, r) \backslash D$ is a compact set, so that we can consider the $\mathcal{L}$-harmonic measure $\omega_{\mathcal{L}}(\partial B(x, 2 r), B(x, 2 r) \backslash E)$ in the classical sense. In case $E$ is not compact, we consider $\omega_{\mathcal{L}}(\partial B(x, 2 r), B(x, 2 r) \backslash E)$ in the extended sense, i.e., the right-hand side of $(12.3)$.

Remark 12.5. In view of (1.4), we have

$$
w_{\eta / c_{\mathcal{L}}^{2}}(D) \leq w_{\eta}^{\mathcal{L}}(D) \quad \text { and } \quad w_{\eta / c_{\mathcal{L}}^{2}}^{\mathcal{L}}(D) \leq w_{\eta}(D) .
$$

We shall see in Lemma 12.8 below that the constant $\eta$ is not so important; a different $\eta$ gives a comparable capacitary width.

Applying Lemma 12.2 repeatedly, we obtain the following.

Lemma 12.6. Let $D$ be an open set with $w_{\eta}^{\mathcal{L}}(D)<\infty$. Suppose $x \in D$ and $R>0$. If $k$ is a positive integer such that $R-2 k w_{\eta}^{\mathcal{L}}(D)>0$, then

$$
\sup _{D \cap \bar{B}\left(x, R-2 k w_{\eta}^{\mathcal{L}}(D)\right)} \omega_{\mathcal{L}}(D \cap \partial B(x, R), D \cap B(x, R)) \leq\left(1-A_{16}^{-1} \eta\right)^{k} .
$$

Proof. For simplicity we let

$$
\Omega= \begin{cases}\omega_{\mathcal{L}}(D \cap \partial B(x, R), D \cap B(x, R)) & \text { on } D \cap B(x, R), \\ 0 & \text { on } B(x, R) \backslash D .\end{cases}
$$

Then $\Omega$ is an $\mathcal{L}$-subharmonic function in $B(x, R)$ with $0 \leq \Omega \leq 1$. Let $w_{\eta}^{\mathcal{L}}(D)<$ $\rho<\infty$. It is sufficient to show that if $R-2 k \rho>0$, then

$$
\sup _{\bar{B}(x, R-2 k \rho)} \Omega \leq\left(1-A_{16}^{-1} \eta\right)^{k} .
$$


Let us prove this inequality by induction on $k$. Since it trivially holds for $k=0$, we assume that $k \geq 1$ and (12.4) holds for $k-1$. In view of the maximum principle, it suffices to show that

$$
\sup _{D \cap \partial B(x, R-2 k \rho)} \Omega \leq\left(1-A_{16}^{-1} \eta\right)^{k} .
$$

By definition there exists $r, w_{\eta}^{\mathcal{L}}(D) \leq r<\rho$, such that

$$
\frac{\operatorname{Cap}_{B(y, 2 r)}^{\mathcal{L}}(\bar{B}(y, r) \backslash D)}{\operatorname{Cap}_{B(y, 2 r)}^{\mathcal{L}}(\bar{B}(y, r))} \geq \eta \quad \text { for every } y \in D .
$$

Take an arbitrary point $y \in D \cap \partial B(x, R-2 k \rho)$. In view of Lemma 12.2 with $E=\bar{B}(y, r) \backslash D$, we have

$$
\omega_{\mathcal{L}}^{y}(\partial B(y, 2 r), B(y, 2 r) \cap D) \leq \omega_{\mathcal{L}}^{y}(\partial B(y, 2 r), B(y, 2 r) \backslash E) \leq 1-A_{16}^{-1} \eta .
$$

Since $\partial B(y, 2 r) \subset \bar{B}(x, R-2(k-1) \rho)$, it follows from the maximum principle and (12.4) with $k-1$ in place of $k$ that

$$
\Omega \leq\left(1-A_{16}^{-1} \eta\right)^{k-1} \omega_{\mathcal{L}}(\partial B(y, 2 r), B(y, 2 r) \cap D)
$$

in $B(y, 2 r) \cap D$. Evaluating at $y$, we obtain

$$
\Omega(y) \leq\left(1-A_{16}^{-1} \eta\right)^{k} .
$$

Since $y \in D \cap \partial B(x, R-2 k \rho)$ is arbitrary, we have (12.5). The lemma is proved.

Theorem 12.7 (Proposition 2 in [1] and Lemma 1 in [2]). Let $D$ be an open set, $x \in D$ and $R>0$. Then

$$
\omega_{\mathcal{L}}^{x}(D \cap \partial B(x, R), D \cap B(x, R)) \leq A_{5} \exp \left(-\frac{A_{6} R}{w_{\eta}^{\mathcal{L}}(D)}\right),
$$

where positive constants $A_{5}$ and $A_{6}$ depend only on $n, c_{\mathcal{L}}$ and $\eta$.

Proof. Let $\Omega=\omega_{\mathcal{L}}(D \cap \partial B(x, R), D \cap B(x, R))$. Since $0 \leq \Omega \leq 1$, the required inequality trivially holds with $A_{5}=1$ in case $w_{\eta}^{\mathcal{L}}(D)=\infty$. So, we may assume that $w_{\eta}^{\mathcal{L}}(D)<\infty$. Let $k$ be the integer such that $2 k w_{\eta}^{\mathcal{L}}(D)<R \leq 2(k+1) w_{\eta}^{\mathcal{L}}(D)$. Then Lemma 12.6 gives

$$
\begin{aligned}
\Omega(x) & \leq\left(1-A_{16}^{-1} \eta\right)^{k}=\exp \left(-k \log \frac{1}{1-A_{16}^{-1} \eta}\right) \\
& \leq \exp \left(-\left(\frac{R}{2 w_{\eta}^{\mathcal{L}}(D)}-1\right) \log \frac{1}{1-A_{16}^{-1} \eta}\right),
\end{aligned}
$$

which implies the required inequality with

$$
A_{5}=\frac{1}{1-A_{16}^{-1} \eta} \quad \text { and } \quad A_{6}=\frac{1}{2} \log \frac{1}{1-A_{16}^{-1} \eta} .
$$

Lemma 12.8 (Proposition 2 in [1]). Let $0<\eta<\eta^{\prime}<1$. Then

$$
w_{\eta}^{\mathcal{L}}(D) \leq w_{\eta^{\prime}}^{\mathcal{L}}(D) \leq A w_{\eta}^{\mathcal{L}}(D) \text { for every open set } D,
$$

where $A>1$ depends only on $\eta, \eta^{\prime}, n$, and $c_{\mathcal{L}}$. 
Proof. By definition the first inequality is obvious. Let us prove the second inequality. In view of Lemma 12.1, we find an integer $N \geq 2$ depending only on $n$ and $c_{\mathcal{L}}$ such that

$$
\frac{\operatorname{Cap}_{B(x, 2 R)}^{\mathcal{L}}\left(\bar{B}\left(x,\left(1-N^{-1}\right) R\right)\right)}{\operatorname{Cap}_{B(x, 2 R)}^{\mathcal{L}}(\bar{B}(x, R))} \geq \sqrt{\eta^{\prime}}
$$

uniformly for $x \in \mathbb{R}^{n}$ and $R>0$. We may assume that $w_{\eta}^{\mathcal{L}}(D)<\infty$. Take an integer $k>2$ so large that $\left(1-A_{16}^{-1} \eta\right)^{k} \leq 1-\sqrt{\eta^{\prime}}$. We apply Lemma 12.6 to $x \in D$ and $R=2 N k \rho$, where we write $\rho=w_{\eta}^{\mathcal{L}}(D)$ for simplicity. We have

$$
\sup _{D \cap \bar{B}(x, R-2 k \rho)} \omega_{\mathcal{L}}(D \cap \partial B(x, R), D \cap B(x, R)) \leq\left(1-A_{16}^{-1} \eta\right)^{k} \leq 1-\sqrt{\eta^{\prime}} .
$$

Let $E=\bar{B}(x, R) \backslash D$. Then the maximum principle yields

$\omega_{\mathcal{L}}(\partial B(x, 2 R), B(x, 2 R) \backslash E) \leq \omega_{\mathcal{L}}(D \cap \partial B(x, R), D \cap B(x, R)) \quad$ on $D \cap B(x, R)$,

so that

$$
\omega_{\mathcal{L}}(\partial B(x, 2 R), B(x, 2 R) \backslash E) \leq 1-\sqrt{\eta^{\prime}} \quad \text { on } \bar{B}(x, R-2 k \rho),
$$

where we use the convention $\omega_{\mathcal{L}}(\partial B(x, 2 R), B(x, 2 R) \backslash E)=0$ on $E$. Hence, the first assertion of Lemma 12.2 with $R-2 k \rho$ and $2 R$ in place of $r$ and $R$ gives

$$
1-\frac{\operatorname{Cap}_{B(x, 2 R)}^{\mathcal{L}}(E)}{\operatorname{Cap}_{B(x, 2 R)}^{\mathcal{L}}(\bar{B}(x, R-2 k \rho))} \leq 1-\sqrt{\eta^{\prime}},
$$

so that

$$
\frac{\operatorname{Cap}_{B(x, 2 R)}^{\mathcal{L}}(E)}{\operatorname{Cap}_{B(x, 2 R)}^{\mathcal{L}}(\bar{B}(x, R-2 k \rho))} \geq \sqrt{\eta^{\prime}} .
$$

Multiplying the inequality and (12.6), we obtain

$$
\frac{\operatorname{Cap}_{B(x, 2 R)}^{\mathcal{L}}(E)}{\operatorname{Cap}_{B(x, 2 R)}^{\mathcal{L}}(\bar{B}(x, R))} \geq \eta^{\prime},
$$

as $R-2 k \rho=\left(1-N^{-1}\right) R$. Since $x \in D$ is arbitrary, we have $w_{\eta^{\prime}}^{\mathcal{L}}(D) \leq R=$ $2 N k \rho=2 N k w_{\eta}^{\mathcal{L}}(D)$, which implies the required inequality.

Proof of Lemma E. Combine Remark 12.5, Lemma 12.8 and Theorem 12.7.

Theorem 12.9 (Theorem 1 in [1]). There exists a constant $A>1$ depending only on $c_{\mathcal{L}}, \eta$ and $n$ such that

$$
A^{-1} w_{\eta}^{\mathcal{L}}(D)^{2} \leq \sup _{x \in D} \int_{D} G_{D}^{\mathcal{L}}(x, y) d y \leq A w_{\eta}^{\mathcal{L}}(D)^{2}
$$

for every open set $D$ with Green function $G_{D}^{\mathcal{L}}$. 
Proof. In view of the monotonicity of the Green function and the monotone convergence theorem, we may assume that $D$ is a bounded open set. Let

$$
v=\int_{D} G_{D}^{\mathcal{L}}(\cdot, y) d y
$$

Since $D$ is bounded, we have $\|v\|_{\infty}<\infty$. First, let us prove the second inequality of (12.7). We may assume that $w_{\eta}^{\mathcal{L}}(D)<\infty$. By definition we find $r, w_{\eta}^{\mathcal{L}}(D) \leq$ $r<2 w_{\eta}^{\mathcal{L}}(D)$, such that

$$
\frac{\operatorname{Cap}_{B(x, 2 r)}^{\mathcal{L}}(\bar{B}(x, r) \backslash D)}{\operatorname{Cap}_{B(x, 2 r)}^{\mathcal{L}}(\bar{B}(x, r))} \geq \eta \quad \text { for every } x \in D .
$$

For a moment we fix $x \in D$ and let $B=B(x, r), B^{*}=B(x, 2 r)$, and $E=\bar{B} \backslash D$ for simplicity. Then $\operatorname{Cap}_{B^{*}}^{\mathcal{L}}(E) / \operatorname{Cap}_{B^{*}}^{\mathcal{L}}(\bar{B}) \geq \eta$. In view of Lemma 12.2 we have

$$
\omega_{\mathcal{L}}^{x}\left(D \cap \partial B^{*}, D \cap B^{*}\right) \leq \omega_{\mathcal{L}}^{x}\left(\partial B^{*}, B^{*} \backslash E\right) \leq 1-A_{16}^{-1} \eta,
$$

where $A_{16}>1$ depends only on $n$ and $c_{\mathcal{L}}$. Let

$$
u=\int_{B^{*}} G_{B^{*}}^{\mathcal{L}}(\cdot, y) d y \quad \text { and } \quad v_{K}=\int_{K} G_{D}^{\mathcal{L}}(\cdot, y) d y
$$

for a compact subset $K \subset D$. It is easy to see that $v_{K}-u$ is $\mathcal{L}$-subharmonic in $D \cap B^{*}$ and $v_{K}=0$ q.e. on $\partial D$. Hence the maximum principle yields

$$
v_{K}-u \leq\left\|v_{K}\right\|_{\infty} \omega_{\mathcal{L}}\left(D \cap \partial B^{*}, D \cap B^{*}\right) \text { on } D \cap B^{*},
$$

so that

$$
\begin{aligned}
v_{K}(x) & \leq u(x)+\left\|v_{K}\right\|_{\infty} \omega_{\mathcal{L}}^{x}\left(D \cap \partial B^{*}, D \cap B^{*}\right) \\
& \leq u(x)+\left\|v_{K}\right\|_{\infty}\left(1-A_{16}^{-1} \eta\right) \leq A r^{2}+\left\|v_{K}\right\|_{\infty}\left(1-A_{16}^{-1} \eta\right)
\end{aligned}
$$

by (12.8), and by an easy estimate $u(x) \leq A r^{2}$ with $A$ depending only on $n$ and $c_{\mathcal{L}}$. Taking the supremum with respect to $x \in D$, we obtain

$$
\left\|v_{K}\right\|_{\infty} \leq A A_{16} \eta^{-1} r^{2} \leq 4 A A_{16} \eta^{-1} w_{\eta}^{\mathcal{L}}(D)^{2} .
$$

Letting $K \uparrow D$, we obtain $v_{K} \uparrow v$ by the monotone convergence theorem, and hence, the second inequality of (12.7).

Second, let us prove the first inequality of (12.7). Let $x \in D$ and $R>0$ to be determined later. This time, we let $B=B(x, R), B^{*}=B(x, 2 R)$ and $E=\bar{B} \backslash D$ for simplicity. We shall compare $v$ and the Green potential

$$
U=\int_{B} G_{B}^{\mathcal{L}}(\cdot, y) d y
$$

Observe that

$$
U \leq A R^{2} \quad \text { on } B ; \quad U \geq A_{17} R^{2} \quad \text { on } \bar{B}(x, R / 2)
$$


with $A$ and $A_{17}$ depending only on $c_{\mathcal{L}}$ and $n$. We may assume that $\|v\|_{\infty}<\infty$. Choose $R>0$ such that

$$
A_{17} R^{2}=2\|v\|_{\infty}
$$

Observe that $U-v$ is $\mathcal{L}$-harmonic in $D \cap B$. By the maximum principle and (12.9)

$$
\begin{aligned}
U-v & \leq \sup _{E} U \cdot \omega_{\mathcal{L}}(\partial E, B \backslash E)=\sup _{E} U \cdot\left(1-\omega_{\mathcal{L}}(D \cap \partial B, B \backslash E)\right) \\
& \leq A R^{2}\left(1-\omega_{\mathcal{L}}\left(\partial B^{*}, B^{*} \backslash E\right)\right) \quad \text { on } D \cap B,
\end{aligned}
$$

since $\partial(D \cap B) \subset(B \cap \partial D) \cup(D \cap \partial B) \subset E \cup \partial B$, and since $U=0$ on $\partial B$. Taking the infimum over $\bar{B}(x, R / 2)$, we obtain from Lemma 12.2 that

$\inf _{\bar{B}(x, R / 2)} U-\|v\|_{\infty} \leq A R^{2}\left(1-\sup _{\bar{B}(x, R / 2)} \omega_{\mathcal{L}}\left(\partial B^{*}, B^{*} \backslash E\right)\right) \leq A R^{2} \frac{\operatorname{Cap}_{B^{*}}^{\mathcal{L}}(E)}{\operatorname{Cap}_{B^{*}}^{\mathcal{L}}(\bar{B}(x, R / 2))}$.

Hence, by (12.9) and (12.10),

$$
A_{17} R^{2}-\frac{A_{17} R^{2}}{2} \leq A R^{2} \frac{\operatorname{Cap}_{B^{*}}^{\mathcal{L}}(E)}{\operatorname{Cap}_{B^{*}}^{\mathcal{L}}(\bar{B}(x, R / 2))}
$$

Dividing by $A R^{2}$, we obtain

$$
\frac{\operatorname{Cap}_{B^{*}}^{\mathcal{L}}(E)}{\operatorname{Cap}_{B^{*}}^{\mathcal{L}}(\bar{B}(x, R / 2))} \geq \frac{A_{17}}{2 A},
$$

so that, by Lemma 12.1,

$$
\frac{\operatorname{Cap}_{B^{*}}^{\mathcal{L}}(E)}{\operatorname{Cap}_{B^{*}}^{\mathcal{L}}(\bar{B}(x, R))}=\frac{\operatorname{Cap}_{B^{*}}^{\mathcal{L}}(E)}{\operatorname{Cap}_{B^{*}}^{\mathcal{L}}(\bar{B}(x, R / 2))} \cdot \frac{\operatorname{Cap}_{B^{*}}^{\mathcal{L}}(\bar{B}(x, R / 2))}{\operatorname{Cap}_{B^{*}}^{\mathcal{L}}(\bar{B}(x, R))} \geq \frac{A_{17} \kappa(1 / 2)}{2 A} .
$$

Thus

$$
\frac{\operatorname{Cap}_{B^{*}}^{\mathcal{L}}(\bar{B}(x, R) \backslash D)}{\operatorname{Cap}_{B^{*}}^{\mathcal{L}}(\bar{B}(x, R))} \geq \eta^{\prime} \quad \text { with } \eta^{\prime}=\frac{A_{17} \kappa(1 / 2)}{2 A} .
$$

Since $x \in D$ is arbitrary, we have $w_{\eta^{\prime}}^{\mathcal{L}}(D) \leq R$ and so $w_{\eta}^{\mathcal{L}}(D) \leq A R$ by Lemma 12.8 . Hence $w_{\eta}^{\mathcal{L}}(D)^{2} \leq A\|v\|_{\infty}$ by (12.10). The proof is complete.

Proof of Lemma D. Combine Remark 12.5, Lemma 12.8 and Theorem 12.9.

\section{References}

[1] Aikawa, H.: Norm estimate of Green operator, perturbation of Green function and integrability of superharmonic functions. Math. Ann. 312 (1998), no. 2, 289-318.

[2] Aikawa, H.: Boundary Harnack principle and Martin boundary for a uniform domain. J. Math. Soc. Japan 53 (2001), no. 1, 119-145.

[3] Aikawa, H.: Potential-theoretic characterizations of nonsmooth domains. Bull. London Math. Soc. 36 (2004), no. 4, 469-482. 
[4] Aikawa, H.: Boundary Harnack principle and the quasihyperbolic boundary condition. In Sobolev spaces in mathematics. II, 19-30. Int. Math. Ser. (NY) 9, Springer, New York, 2009.

[5] Aikawa, H.: Extended Harnack inequalities with exceptional sets and a boundary Harnack principle. J. Anal. Math. 124 (2014), 83-116.

[6] AnconA, A.: On strong barriers and an inequality of Hardy for domains in $\mathbb{R}^{n}$. J. London Math. Soc. (2) 34 (1986), no. 2, 274-290.

[7] Armitage, D. H. And Gardiner, S. J.: Classical potential theory. Springer Monographs in Mathematics, Springer-Verlag London, London, 2001.

[8] BAÑuelos, R.: Intrinsic ultracontractivity and eigenfunction estimates for Schrödinger operators. J. Funct. Anal. 100 (1991), no. 1, 181-206.

[9] Bañuelos, R. AND Davis, B.: A geometrical characterization of intrinsic ultracontractivity for planar domains with boundaries given by the graphs of functions. Indiana Univ. Math. J. 41 (1992), no. 4, 885-913.

[10] Bass, R. F.: Probabilistic techniques in analysis. Probability and its Applications (New York), Springer-Verlag, New York, 1995.

[11] Bass, R.F. AND Burdzy, K.: A boundary Harnack principle in twisted Hölder domains. Ann. of Math. (2) 134 (1991), no. 2, 253-276.

[12] Bass, R. F. And Burdzy, K.: Lifetimes of conditioned diffusions. Probab. Theory Related Fields 91 (1992), no. 3-4, 405-443.

[13] VAN DEN Berg, M.: Estimates for the torsion function and Sobolev constants. Potential Anal. 36 (2012), no. 4, 607-616.

[14] van den Berg, M. and Carroll, T.: Hardy inequality and $L^{p}$ estimates for the torsion function. Bull. Lond. Math. Soc. 41 (2009), no. 6, 980-986.

[15] Cipriani, F.: Intrinsic ultracontractivity of Dirichlet Laplacians in nonsmooth domains. Potential Anal. 3 (1994), no. 2, 203-218.

[16] Davies, E. B.: Heat kernels and spectral theory. Cambridge Tracts in Mathematics 92, Cambridge University Press, Cambridge, 1989.

[17] Davies, E. B. And Simon, B.: Ultracontractivity and the heat kernel for Schrödinger operators and Dirichlet Laplacians. J. Funct. Anal. 59 (1984), no. 2, 335-395.

[18] Davis, B.: Intrinsic ultracontractivity and the Dirichlet Laplacian. J. Funct. Anal. 100 (1991), no. 1, 162-180.

[19] Doob, J. L.: Classical potential theory and its probabilistic counterpart. Reprint of the 1984 edition. Classics in Mathematics, Springer-Verlag, Berlin, 2001.

[20] Fabes, E. B. And Stroock, D. W.: A new proof of Moser's parabolic Harnack inequality using the old ideas of Nash. Arch. Rational Mech. Anal. 96 (1986), no. 4, $327-338$.

[21] Gyrya, P. and Saloff-Coste, L.: Neumann and Dirichlet heat kernels in inner uniform domains. Astérisque 336 (2011), viii+144 pp.

[22] Hansen, W.: Simple counterexamples to the 3G-inequality. Expo. Math. 24 (2006), no. $1,97-102$.

[23] Kemper, J. T.: Boundary properties of minimal harmonic functions. Proc. Amer. Math. Soc. 60 (1976), 193-196. 
[24] Kim, P. And Song, R.: Intrinsic ultracontractivity of non-symmetric diffusion semigroups in bounded domains. Tohoku Math. J. (2) 60 (2008), no. 4, 527-547.

[25] Lieb, E. H.: On the lowest eigenvalue of the Laplacian for the intersection of two domains. Invent. Math. 74 (1983), no. 3, 441-448.

[26] Maz'ya, V. And Shubin, M.: Can one see the fundamental frequency of a drum? Lett. Math. Phys. 74 (2005), no. 2, 135-151.

[27] Méndez-Hernández, P. J.: Toward a geometric characterization of intrinsic ultracontractivity for Dirichlet Laplacians. Michigan Math. J. 47 (2000), no. 1, 79-99.

[28] Murata, M.: Nonuniqueness of the positive Dirichlet problem for parabolic equations in cylinders. J. Funct. Anal. 135 (1996), no. 2, 456-487.

[29] Murata, M.: Semismall perturbations in the Martin theory for elliptic equations. Israel J. Math. 102 (1997), 29-60.

[30] Murata, M.: Martin boundaries of elliptic skew products, semismall perturbations, and fundamental solutions of parabolic equations. J. Funct. Anal. 194 (2002), no. 1, 53-141.

[31] Murata, M.: Uniqueness theorems for parabolic equations and Martin boundaries for elliptic equations in skew product form. J. Math. Soc. Japan 57 (2005), no. 2, 387-413.

[32] Murata, M. And Suzuki, N.: A parabolic approach to Martin boundaries for elliptic equations in skew product form. Potential Analysis 40 (2014), no. 3, 279-305.

[33] Ouhabaz, E. M. And Wang, F.-Y.: Sharp estimates for intrinsic ultracontractivity on $C^{1, \alpha}$-domains. Manuscripta Math. 122 (2007), no. 2, 229-244.

[34] Persson, A.: Bounds for the discrete part of the spectrum of a semi-bounded Schrödinger operator. Math. Scand. 8 (1960), 143-153.

[35] Smith, W. and Stegenga, D. A.: Hölder domains and Poincaré domains. Trans. Amer. Math. Soc. 319 (1990), no. 1, 67-100.

[36] Smith, W. and Stegenga, D. A.: Exponential integrability of the quasi-hyperbolic metric on Hölder domains. Ann. Acad. Sci. Fenn. Ser. A I Math. 16 (1991), no. 2, 345-360.

[37] Souplet, P.: Decay of heat semigroups in $L^{\infty}$ and applications to nonlinear parabolic problems in unbounded domains. J. Funct. Anal. 173 (2000), no. 2, 343-360.

[38] Watson, N. A.: Introduction to heat potential theory. Mathematical Surveys and Monographs 182, American Mathematical Society, Providence, RI, 2012.

Received September 4, 2013; revised April 7, 2014.

Hiroaki Aikawa: Department of Mathematics, Hokkaido University, Sapporo 0600810, Japan.

E-mail: aik@math.sci.hokudai.ac.jp

This work was supported in part by JSPS KAKENHI Grant Numbers 20244007, 25287015 and 25610017. Some part of the paper was written during the stay of the author at the Institut Mittag-Leffler (Djursholm, Sweden) in Fall 2013. Support by the Institut Mittag-Leffler is gratefully acknowledged. 